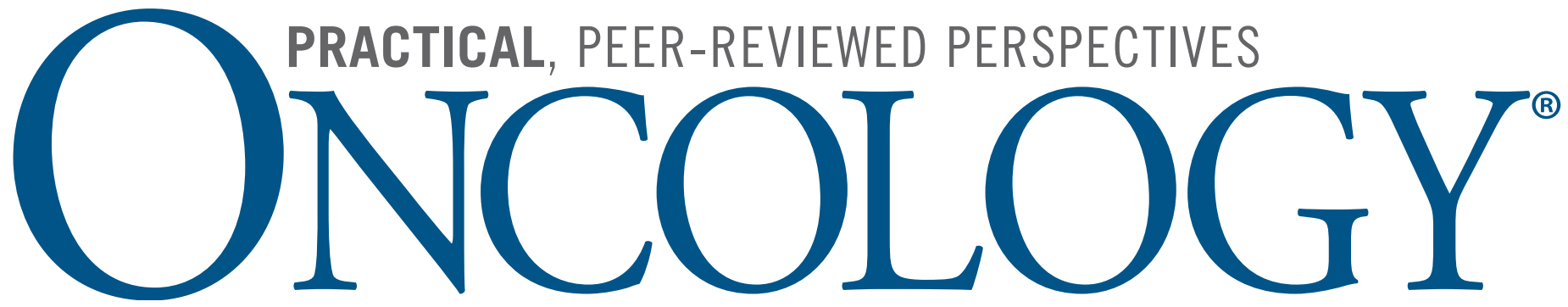

AUGUST 2020 | Vol 34 - No 8

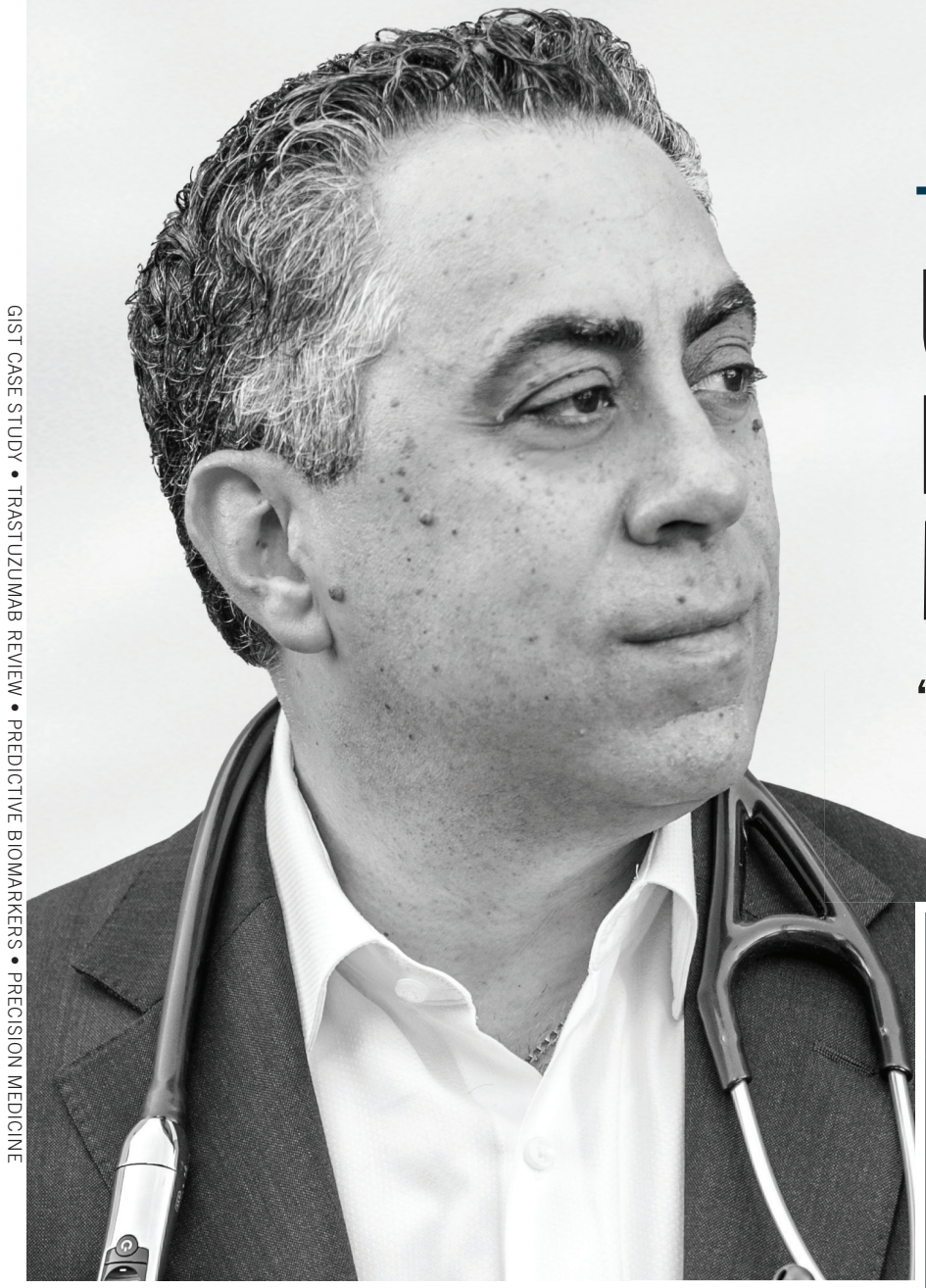

GU Cancer Experts Develop and Create New Guideline for Advanced Prostate Cancer

Hematologic Malignancies Changing Landscape in MDS Guillermo Garcia-Manero, MD

TANIOS S. BEKAII-SAAB, MD, ON Ushering in the Era of Precision Medicine

"Next-generation sequencing should become a right, not a privilege."

Women's Cancer Optimizing the Duration of

Trastuzumab Melissa A. Taylor, MD; Kristina F. Byers, PharmD;

and Jane L. Meisel, MD

Immunotherapy Predictive Biomarkers for

Immunotherapy Response Beyond PD-1/PD-L1

Sourat Darabi, PhD, MS; David R. Braxton, MD; Burton L. Eisenberg, MD; and

Michael J. Demeure, MD, MBA

Lung Cancer The New Era in Lung Cancer Care 


\section{When the path forward in MCRPC is unclear, the Oncotype DX AR-VH Nucleus Detect ${ }^{\circledR}$ test can help navigate.}

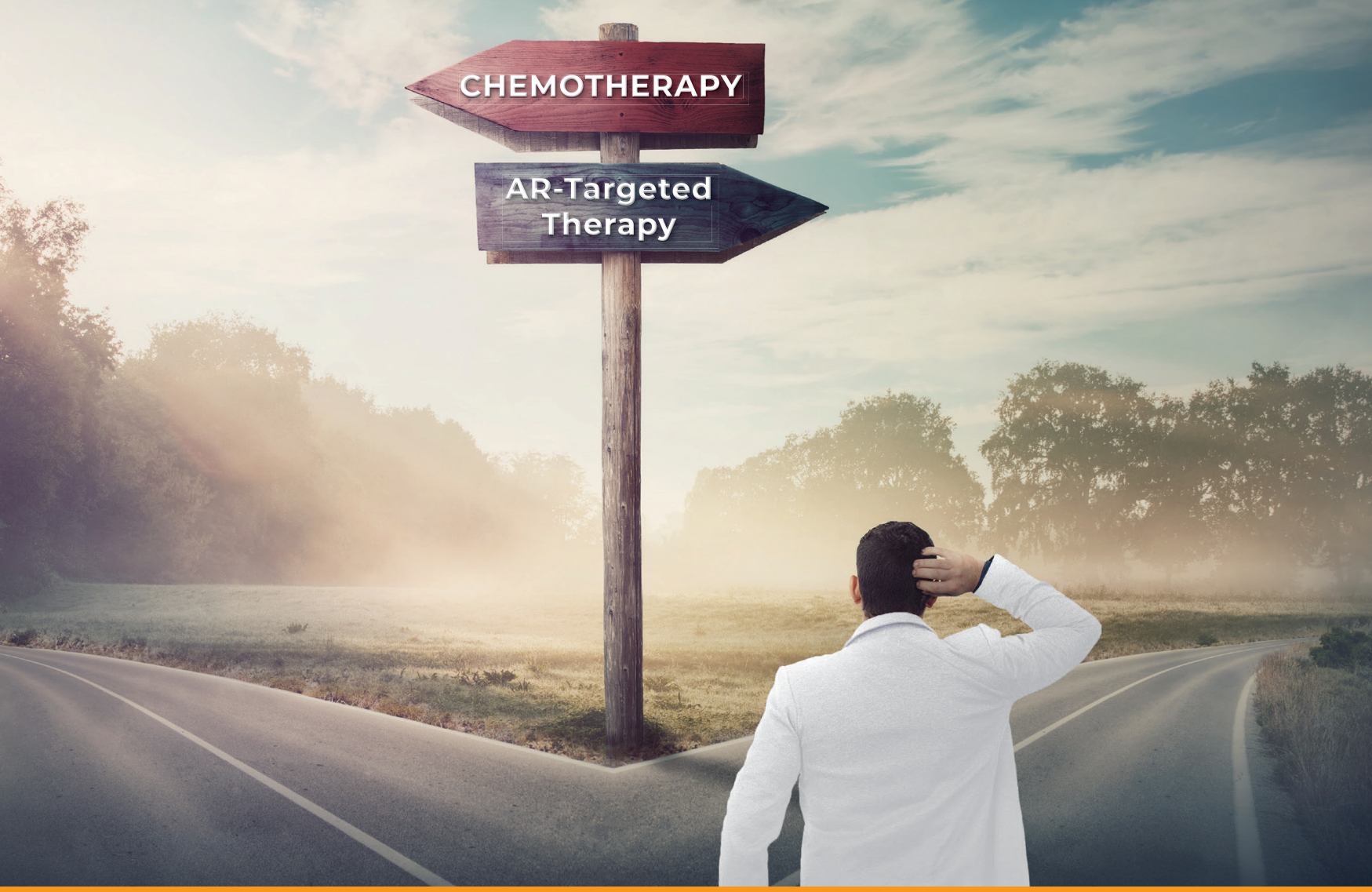

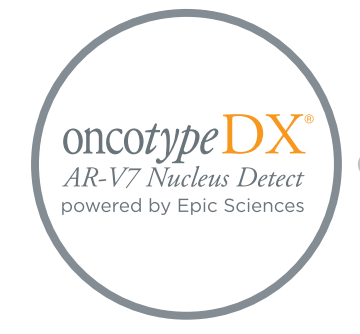

Order the test after a patient fails an AR-targeted therapy

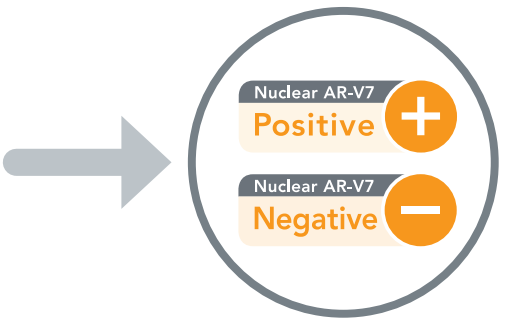

Understand patient's AR-V7 status

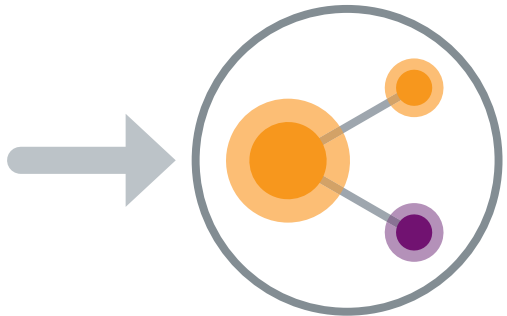

Consider sequential AR-targeted therapy or chemotherapy depending on AR-V7 status 


\section{IN THIS ISSUE}

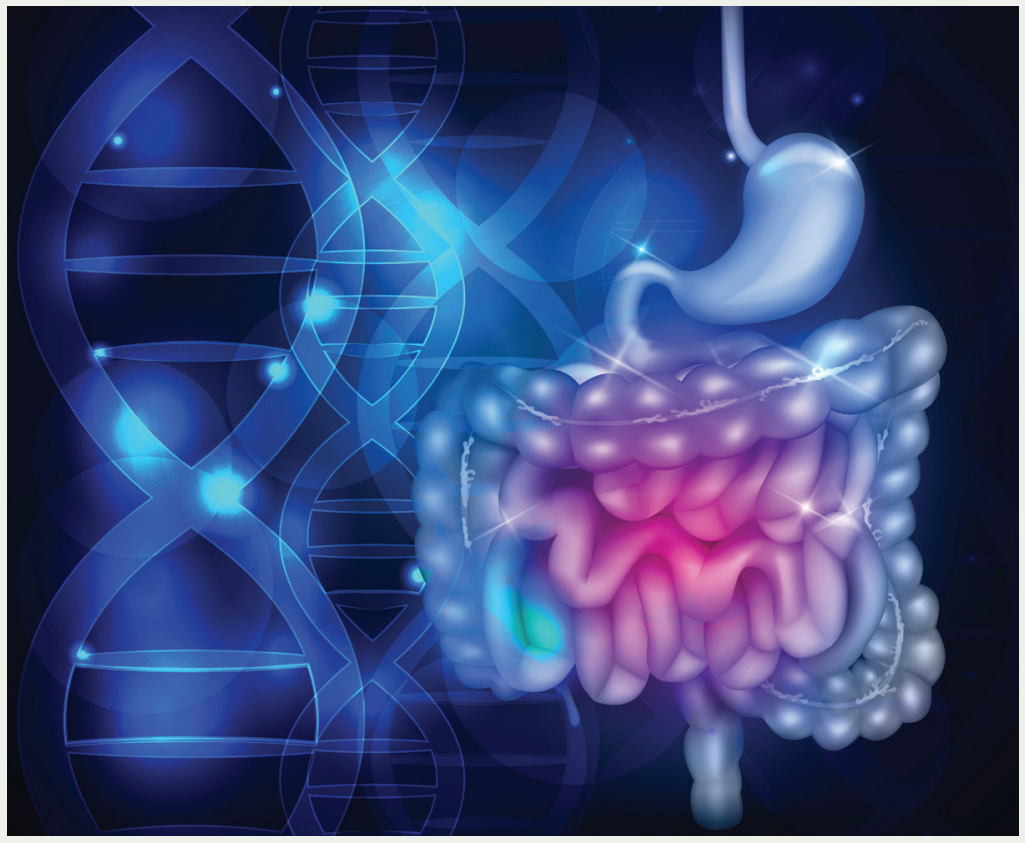

314 GI CANCER: Cover

ONCOLOGY ${ }^{\circledR}$ sat down with Tanios S. Bekaii-Saab, MD, of the Mayo Clinic to discuss next generation sequencing for GI cancers.

\section{Chairman's Letter}

Mike Hennessy Sr

295 Letter to the Reader Cancer: A Patient's "Right"? Or Wrong?

Howard S. Hochster, MD

296 WOMEN'S CANCER: Review

Optimizing the Duration of Trastuzumab: A Fresh Perspective

Melissa A. Taylor, MD; Kristina F. Byers, PharmD; Jane L. Meisel, MD

\section{cancernetwork.com}

Visit CancerNetwork.com, home of the journal ONCOLOGY ${ }^{\circledR}$ and a web destination for oncologists seeking expert peer perspectives, podcasts, and other clinically practical features.

VIDEO

Caron A. Jacobson, MD, on Next Steps for the Phase 2 ZUMA-5 Study

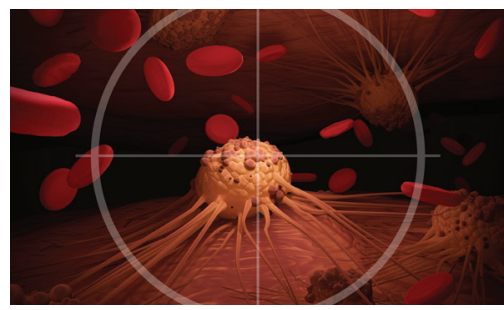

Cancernetwork.com/ZUMA-5_nextsteps

NEWS

Fasting Mimicking Diet May Be Safe, Effective as Adjunct to Chemo for Breast Cancer

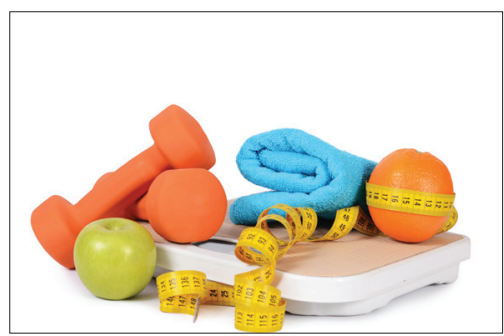

Cancernetwork.com/breastcancer_fasting

NEWS

Study Results Finds

Medicaid-Expanded States

Diagnose Breast Cancer at

Earlier Stage

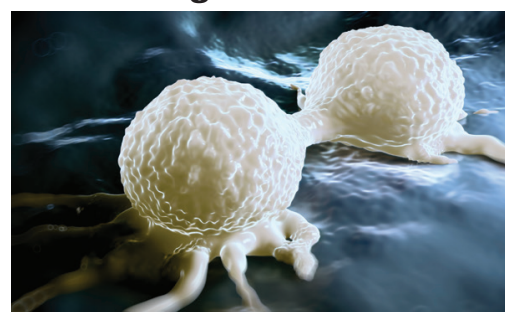

Cancernetwork.com/Medicaid_diagnosis

Table of Contents continued on page 292 


\section{IN THIS ISSUE}

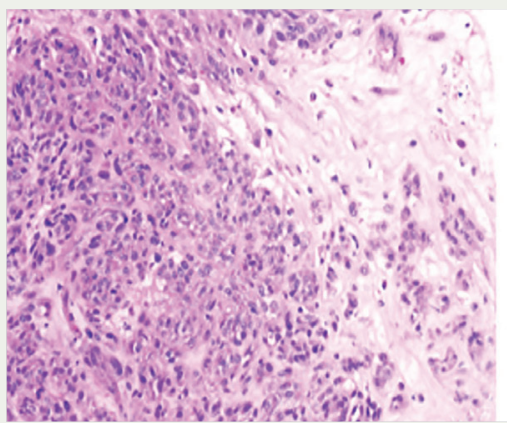

LUNG CANCER: Q\&A 302 The New Era in Lung Cancer Care

ONCOLOGY $Y^{\circledR}$ sat down with Mark G. Kris, MD, of Memorial Sloan Kettering Cancer Center to discuss the recent trends in lung cancer treatment.

\section{GU CANCER: Guidelines} 305 Experts Develop New Guideline for Advanced Prostate Cancer

Jason M. Broderick

\section{GI CANCER: Case Study 307 Locally Advanced Gastrointestinal Stromal Tumor in a 33-Year- Old Woman Seeking to Conceive}

Mehmet Sitki Copur, MD; Alison M. Cushman-Vokoun, MD, PhD; Abigail Delaney, MD; James Padussis, MD; Whitney Wedel, MD; Scott Lauer, MD; Geoffrey A. Talmon, MD

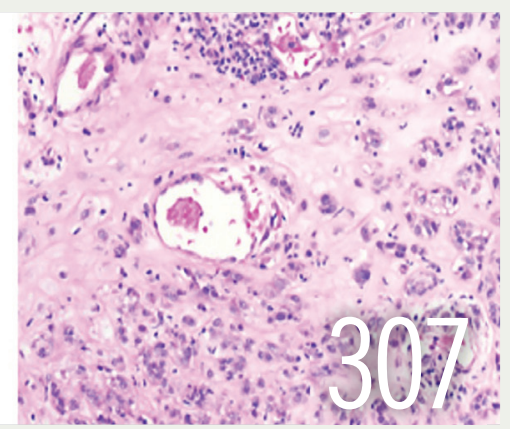

GU Cancer: Clinical Quandaries 317 Challenges of Treating a Patient With Advanced Prostate Cancer During the COVID-19 Pandemic

Lucia Carril-Ajuria, MD; Yuly A Remolina-Bonilla, MD; Alberto Carretero-González, MD; Maricruz Martín-Soberón, MD; Daniel Castellano, MD; Christianne Bourlon, MD; Guillermo de Velasco, MD, PhD; Maria T Bourlon, MD

IMMUNOTHERAPY: Review 321 Predictive Biomarkers for Immunotherapy Response Beyond

\section{PD-1/PD-L1}

Sourat Darabi, PhD, MS; David R. Braxton, MD; Burton L. Eisenberg, MD; and Michael J. Demeure, MD, MBA
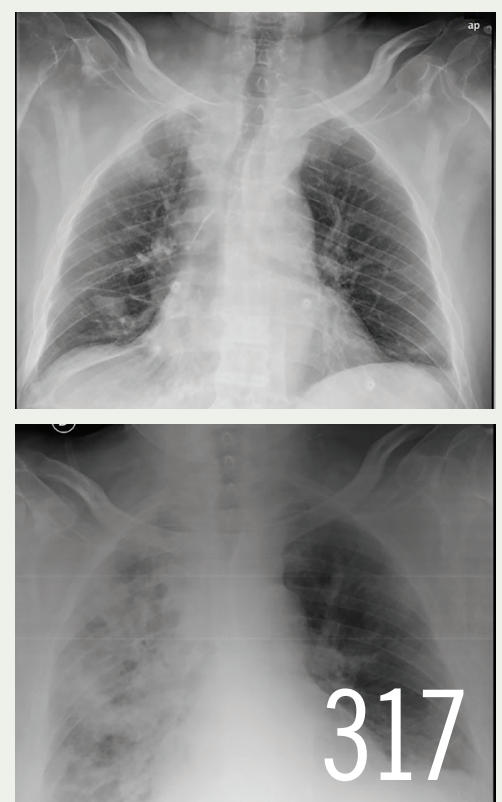

TELEHEALTH: Medical Economics ${ }^{\circledR}$ 328 Five Ways to Prepare Your Patients for Their First Telemedicine Visit

Peter Alperin, MD

\section{HEMATOLOGICAL MALIGNANCIES: Continuing Education 329 Changing Landscape in MDS}

Guillermo Garcia-Manero, MD

Published in affiliation with

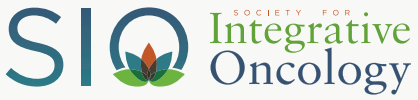

JPR $\mathrm{R}^{\bullet}$ the editors are PLeAsed to announce the availability of our new parent company's continuing education activities. gotoper.com We've picked this one especially for our ONCOLOGY ${ }^{\circledR}$ readers. Go to: https://bit.ly/2IRAknZ guage appears within the publication: "All statements, including product claims, are those of the person or organization making the statement or claim. The publisher does not adopt any such statement or claim as its own, and any such statement or claim does not necessarily reflect the opinion of the publisher."

Advertiser and advertising agency accept and assume liability for all content (including text, representations, illustrations, opinions and facts) of advertisements printed, and also assume responsibility for any claims made against the publisher arising from or related to such advertisements. In the event that lega action or a claim is made against the publisher arising from or related to such advertisements, advertiser and advertising agency agree to fully defend, indemnify and hold harmless the publisher, and to pay anyjudgment, expenses and legal fees incurred by the publisher as a result of said legal action or claim. The publisher reserves the right to reject any advertising which he feels is not in keeping with the publication's standards.

Publisher is not liable for delays in delivery and/or non-delivery in the event of an act of God, action by any government or quasi-governmental entity, fire, flood, insurrection, riot, explosion, embargo, strikes (whether legal or illegal), labor or materia shortage transportation interuetion of any kind, work sow-down, or any condition beyond the control of publisher affecting production or delivery in any manner.
ONCOLOGY (ISSN 0890-9091) is published monthly by MultiMedia Healthcare UC, 2 Clarke Drive, Suite 100 Cranbury, NN 08512. Annual subscription rates: US, \$199 and Canada, \$219; students and nurses, \$96; international, $\$ 249$. Single copies: $\$ 20$ each. Institutional US, $\$ 299$; Canada, $\$ 329$; international, $\$ 375$. Periodicals postage paid at Trenton, $\mathrm{N}$ and at additional mailing offices. POSTMASTER: Please send address changes to Oncology PO Box 457, Cranbury NN 08512-0457, USA. Publications Mail Agreement № 40612608. Return Undeliverable Canadian Addresses to: IMEX Global Solutions, PO Box 25542 London ON N6C 6B2. Canadian G.S.T number: R-124213133RT001. Printed in U.S.A.

For address changes, please notify the Circulation Department by visiting www.surveymonkey.com/s/subscriptions, or by mail to ONCOLOGY ( 2020 MJH Life Sciences, PO Box 457, Cranbury N 08512-0457. Send old address, new address and attach a copy of mail label, if possible. 


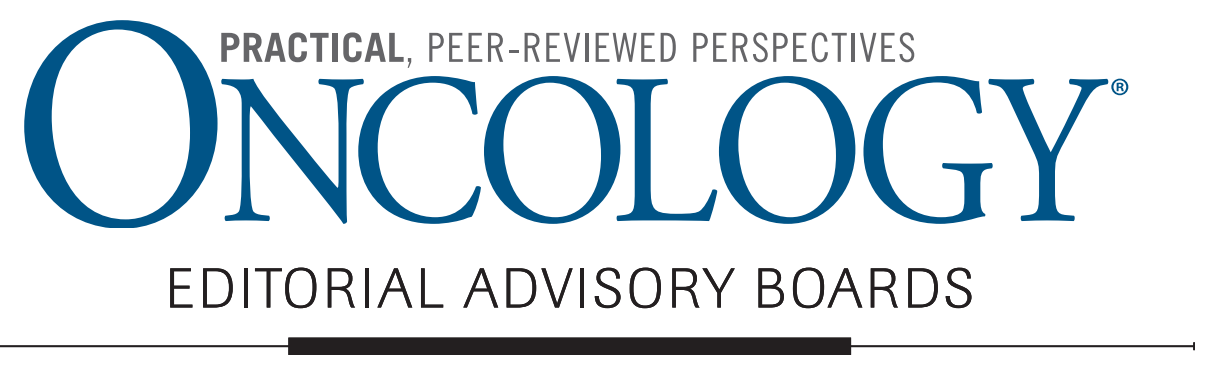

MISSION

STATEMENT

$O N C O L O G Y^{\circledR}$ and its website, CancerNetwork.com, provide oncologists with the practical, timely, clinical information they need to deliver the highest level of care to their patients. Expert authors and peer review ensure the quality of ONCOLOGY ${ }^{\circledR}$ and CancerNetwork.com's articles and features. Focused discussions capture key clinical take-aways for application in today's time-constrained practice environment.

\section{EDITORS-IN-CHIEF}

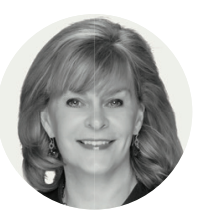

Julie M. Vose, MD, MBA Omaha, NE
GASTROINTESTINAL CANCER

Tanios S. Bekaii-Saab, MD, Phoenix, AZ

HEAD AND NECK CANCER

Eric J. Sherman, MD, New York, NY
HEMATOLOGIC MALIGNANCIES

C. Ola Landgren, MD, PhD, New York, NY

LUNG CANCER

Joshua M. Bauml, MD, Philadelphia, PA

\section{BOARD MEMBERS}

\section{BREAST CANCER}

William J. Gradishar, MD, FACP, Chicago, IL

Tari King, MD, Boston, MA

Vered Stearns, MD, Baltimore, MD

Melinda L. Telli, MD, Palo Alto, CA

CANCER SURVIVORSHIP

Matthew J. Matasar, MD, MS, New York, NY

COLORECTAL/GASTROINTESTINAL CANCER

Edward Chu, MD, Pittsburgh, PA

Mehmet Sitki Copur, MD, FACP, Omaha, NE

Daniel Haller, MD, Philadelphia, PA

John L. Marshall, MD, Washington, DC

Shubham Pant, MD, Houston, TX

Matthew B. Yurgelun, MD, Boston, MA

Mehmet Sitki Copur, MD, FACP, Omaha, NE

GENITOURINARY CANCER

L. Michael Glodé, MD, FACP, Denver, CO

Paul Mathew, MD, Boston, MA

Elisabeth Heath, MD, FACP, Detroit, MI

William Oh, MD, New York, NY

GYNECOLOGIC ONCOLOGY

Mario M. Leitao Jr, MD, New York, NY

Franco Muggia, MD, New York, NY

\section{HEAD AND NECK CANCER}

Apar K. Ganti, MD, MS, FACP, Omaha, NE

HEALTH ECONOMICS

Nora Janjan, MD, Dallas, TX

HEMATOLOGIC MALIGNANCIES

Danielle M. Brander, MD, Durham, NC

Christopher R. Flowers, MD, Houston, TX

Steven T. Rosen, MD, Duarte, CA

Naval G. Daver, MD, Houston, TX

Ehab L. Atallah, MD, Milwaukee, WI

INFECTIOUS DISEASE

Genovefa Papanicolaou, MD, New York, NY

INTEGRATIVE ONCOLOGY

Ting Bao, MD, New York, NY

Linda Carlson, PhD, RPsych, Calgary, Alberta,

Canada

LUNG CANCER

David S. Ettinger, MD, Baltimore, MD

James L. Mulshine, MD, Chicago, IL

Edward S. Kim, MD, Charlotte, NC

Jennifer W. Carlisle, MD, Atlanta, GA

MELANOMA

Richard D. Carvajal, MD, New York, NY

Jason Luke, MD, FACP, Pittsburgh, PA
NEURO-ONCOLOGY

David A. Reardon, MD, Boston, MA

Stuart A. Grossman, MD, Baltimore, MD

Nicole A. Shonka, MD, Omaha, NE

PEDIATRIC ONCOLOGY

David G. Poplack, MD, Houston, TX

Richard A. Drachtman, MD, New Brunswick, NJ

PROSTATE CANCER

Tomasz M. Beer, MD, Portland, OR

E. David Crawford, MD, Denver, CO

Judd W. Moul, MD, FACS, Durham, NC

PSYCHO-ONCOLOGY

Daniel C. McFarland, Do, New York, NY

Michelle Riba, MD, Ann Arbor, MI

RADIATION ONCOLOGY

Louis Potters, MD, FACR, Hempstead, NY

James B. Yu, MD, MHS, New Haven, CT

SARCOMA

Kenneth Cardona, MD, FACS, Atlanta, GA

SUPPORTIVE AND PALLIATIVE CARE

Thomas J. Smith, MD, FACP, Baltimore, MD

N. Simon Tchekmedyian, MD, Long Beach, CA

SURGICAL ONCOLOGY

Burton L. Eisenberg, MD, Newport Beach, CA

\section{INTERESTED IN SUBMITTING TO ONCOLOGY?}

Please contact senior editor Kevin Wright at KWright@mjhlifesciences.com for submission guidelines or more information. 


\section{Finding the Target}

The era of precision medicine is firmly upon us. With the rise of genetic testing and genomic sequencing, one-size-fits-all chemotherapy treatments have given way to targeted therapies, allowing oncologists to tailor treatment regimens to the mutational status of individual patients.

But questions do remain: Who gets tested? Who pays? What are the barriers to implementing regular genomic sequencing for all patients?

In this issue of ONCOLOGY ${ }^{\circledR}$, we spoke with Tanios S. Bekaii-Saab, MD, of the Mayo Clinic in Phoenix, Arizona, about the importance of genomic sequencing and the need to make genetic testing universal in gastrointestinal (GI) cancers.

“I think every single patient with a GI malignancy... has the right [to be sequenced], and we have to find every way possible to get them sequenced," says Bekaii-Saab. "I need to understand what the rare mutational status of each patient is."

Bekaii-Saab also discusses the rapid improvements in treatment for GI cancers, specifically colorectal cancer, that come from the ability to identify the proper target and proper treatment for each patient. "We're catching up, and we're catching up quickly," Bekaii-Saab says of the use of biologic and immune-based therapies in the field of GI cancer. "And I think in the next 3 to 5 years, we'll probably be ahead of the curve again."

The need for regular testing and sequencing applies to all tumor types, and in this issue, you will also hear from Mark G. Kris, MD, of Memorial Sloan Kettering Cancer Center in New York, New York, on the avalanche of recent FDA approvals of lung cancer treatments-each made possible by the rise of targeted therapies.

Also in this issue, you will read a review of predictive biomarkers for immunotherapy response. With the meteoric rise of immune therapeutics for patients with cancer, the ability to predict and understand responses to these treatments has become essential.

For this month's clinical quandary, you will read about the challenges of treating a patient with advanced prostate cancer during the coronavirus disease 2019 pandemic. How have treatment protocols changed? What can we do to ensure the best possible treatment with the lowest possible risk? Read on to find out.

Within these pages, you will also read a review of the optimal treatment duration of trastuzumab (Herceptin) for patients with breast cancer and a case study of a 33-year-old woman given a diagnosis of a locally advanced GI stromal tumor, and the impact her treatment has had regarding her fertility and potential for bearing children.

I hope you find our journal helpful in caring for your patients through what is likely one of the most challenging times in their lives. As always, thank you for reading.

Mike Hennessy $\mathrm{Sr}$ Chairman and Founder of ONCOLOGY'S parent company, MJH LIfe Sciences"

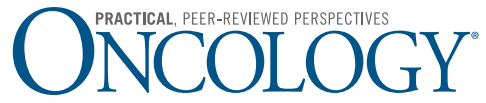

AUGUST 2020 - VOL. 34 - NO. 8

\section{EDITORIAL}

KRISTIE L. KAHL Editorial Director

KEVIN WRIGHT Senior Editor

HANNAH SLATER Assistant Editor

MATTHEW FOWLER Assistant Editor

JENNIFER POTASH Copy Chief

RACHELLE LALIBERTE, PAUL SILVERMAN Copy Supervisors

CHENEY BALTZ, GEORGINA CARSON, KIRSTY MACKAY Copy Editors

\section{DESIGN \& PRODUCTION}

ROBERT MCGARR Creative Director

KRISTEN MORABITO Art Director

RACHEL KEATLEY Graphic Designer

JONATHAN SEVERN Circulation Director

KEYONNA GRAHAM Production Director

\section{PUBLISHING \& SALES}

BRIAN HAUG Executive Vice President 609-325-4780 • bhaug@mmhgroup.com

MARC MATHEWS Vice President Oncology 609-819-5245•mmathews@mjhlifesciences.com

MICHELLE JANIN Director of Sales

732-429-4316•mjanin@mmhgroup.com

PATRIC PASTORE National Accounts Associate 609-955-1694 • ppastore@mjhlifesciences.com

\section{AUDIENCE DEVELOPMENT}

KELLY KEMPER Audience Development Manager

\section{CORPORATE}

MIKE HENNESSY SR Chairman \& Founder

JACK LEPPING Vice Chairman

MIKE HENNESSY JR President \& CEO

NEIL GLASSER, CPA/CFE Chief Financial Officer

TOM TOLVÉ Executive Vice President, Operations

JOE PETROZIELLO Executive Vice President, Global Medical Affairs and Corporate Development

SILAS INMAN Senior Vice President, Content

JOHN MORICONE Senior Vice President, I.T. \& Enterprise Systems

JoY PUZZO Senior Vice President, Audience Generation \& Product Fulfillment

SHARI LUNDENBERG Vice President, Human Resources \& Administration

CHRIS HENNESSY Vice President, Mergers \& Acquisitions

JEFF BROWN Executive Creative Director, Creative Services

\section{SUBSCRIPTIONS}

888-527-7008

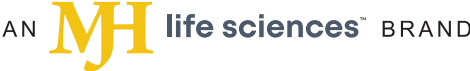




\title{
LETTER TO THE READERS
}

\section{Next-Generation Sequencing Testing in GI Cancer: A Patient's "Right"? Or Wrong?}

\author{
Howard S. Hochster, MD \\ Distinguished Professor of Medicine, Rutgers Robert Wood Johnson Medical School \\ Associate Director for Clinical Research, Rutgers Cancer Institute of New Jersey \\ Director of Oncology Research, RWJBarnabas Health
}

$\mathrm{n}$ this issue of $O N C O L O G Y^{\circledR}$, the

Q\&A with Tanios S. Bekaii-Saab,

$\mathrm{MD}$, raises some interesting questions on next-generation sequencing (NGS) testing. Bekaii-Saab is a national leader in gastrointestinal (GI) cancer, especially hepato-biliary cancer. He has contributed to important studies in GI Oncology through many organizations and especially Academic and Community Cancer Research United, and most importantly, he is a friend.

In this Q\&A (see page 314), he says that NGS testing should be done at diagnosis for all patients with GI cancer, and this is no longer a privilege but a patient's right. Although I agree with the basic premise that NGS will give us the greatest amount of information in a single test, I think this statement needs a little more consideration.

First, at the time of diagnosis, the test is not sent immediately but usually requires the medical oncologist to try to order it after first consulting with the patient, and potentially from another hospital where the surgery was done. First, if an endoscopic biopsy was done, we often find (after considerable chasing) that insufficient tissue remains. Second, this process takes a minimum of 2 weeks, so now the patient is already
4 to 5 weeks post diagnosis, and possibly more if they had surgery with a recovery period. Further waiting is often traumatic for the patient.

Next, we have to consider the utility of NGS testing. Bekaii-Saab mentions microsatellite instability status, which we must be sure to get right. But that is usually done on the biopsy or surgical specimen by immunohistochemistry (IHC) for presence or deficiency of mismatch repair enzymes. We do not need NGS for this. Next is RAS and RAF status. These key enzymes in the growth signaling pathway need to be known to get the regimen correct. But these can be done in 48 hours or less by a polymerase chain reaction test than requires far less DNA. At present, $R A S$ status may be far less consequential than right versus s left sidedness and is only helpful if one wishes to start with an anti-EGFR antibody. $B R A F$ status is quite consequential for both prognosis and treatmentparticularly as the $B R A F$ inhibitors move into first-line therapy. But NGS sequencing is not as fast or cheap as a PCR test. What about HER2? It is so important for both prognosis and treatment with anti-HER2 antibodies (please consider SWOG 1613 trial, now accruing) and is a critical biomarker for those overexpressing.
But seriously, we have been defining HER 2 overexpression by IHC for more than a decade in breast cancer; we do not need NGS for this. We just need to ensure those who are RAS wild type are tested with IHC and fluorescence in situ hybridizationand they will constitute an $8 \%$ subgroup in this setting.

So, what remains? A few important NTRK and similar fusions constituting $1 \%$ of all colon cancer. It's true you could miss a few patients with high tumor mutational burden, but these are mostly POLE mutated and rarer than hen's teeth. Ninety percent of the time we will not find any actionable mutation given our state of knowledge and available therapeutics.

Finally, let's consider the cost of upfront NGS for colon cancer alone. With 40,000 new cases per year of metastatic colon cancer alone, at $\$ 5000$ each for NGS, we would spend \$200 million! The options above cost a fraction of this cost. We can save the money and use it for circulating DNA testing later in the course of therapy when mutations may have evolved. NGS is a very useful test in many circumstances, but in most GI cancers the mutational landscape and therapeutic options limit its utility. 


\section{Optimizing the Duration of Trastuzumab: A Fresh Perspective}

Melissa A. Taylor, MD, Department of Medicine, Emory University School of Medicine Kristina F. Byers, PharmD, Winship Cancer Institute, Emory University

Jane L. Meisel, MD, Winship Cancer Institute, Emory University

\section{Introduction}

Prior to the introduction of trastuzumab, the first targeted anti-HER2 agent, in 1998, patients diagnosed with HER2-positive breast cancer felt like they were being handed a death sentence. Despite treatment with aggressive chemotherapy, their tumors recurred faster, more often spread to brain and liver, and were associated with higher rates of death than HER2-negative tumors. ${ }^{1}$ HER2-positive breast cancer was also more prevalent in younger patients, making the diagnosis even more devastating. ${ }^{2}$ However, in the 1980 s, cancer researcher Axel Ullrich, PhD, and oncologist Dennis Slamon, MD, PhD, recognized that HER2 could be targeted by a small molecule that binds to the receptor on the cell surface and blocks the signal telling the cell to divide. ${ }^{3}$ This small molecule was called trastuzumab, and it eventually completely changed how HER2-positive breast cancer was treated.

The availability of trastuzumab gave much-needed hope to patients diagnosed with this aggressive form of breast cancer. The drug was first approved in the metastatic setting, and then the results of 2 pivotal randomized control trials in 2005 $5^{4-5}$ demonstrated that the administration of trastuzumab in the adjuvant setting decreased the risk of breast cancer recurrence by $50 \%$, leading to its approval in 2006 by the FDA for use as adjuvant therapy for early-stage HER2-positive breast cancer. ${ }^{6}$ These trials showed trastuzumab to be unequivocally effective in the adjuvant setting; however, the optimal duration of treatment was still unknown.

The HERA trial was designed to address the question of adjuvant trastuzumab duration, randomizing patients 1:1:1 to receive 1 year of adjuvant trastuzumab, 2 years of adjuvant trastuzumab, or no adjuvant trastuzumab; then, important disease-related outcomes were compared among groups. ${ }^{7}$ Investigators demonstrated statistically significant improvement in 2-year disease-free survival (DFS) in patients treated with 1 and 2 years of adjuvant trastuzumab compared with patients who received no trastuzumab; however, there was no difference in outcomes for 1 year vs 2 years of trastuzumab. HERA's results led to the adoption of 1 year of adjuvant trastuzumab as the standard of care.

Since that time, the field of antiHER2-targeted therapy has exploded, with the development of multiple targeted agents for use in the advanced and up-front settings. We also have a wealth of options in addition to adjuvant trastuzumab for our patients with the highest-risk early-stage disease. APHINITY (NCT01358877) established the benefit of the addition of a year of adjuvant trastuzumab/pertuzumab for patients with node-positive disease. ${ }^{8}$ The KATHERINE trial (NCT01772472) established the benefit of switching to adjuvant ado-trastuzumab emtansine (T-DM1; Kadcyla) rather than continuing trastuzumab if a pathological complete response is not achieved with neoadjuvant anti-HER2 therapy (albeit with more toxicity than adjuvant trastuzmab). ${ }^{9}$ In addition to these options for intensifying therapy, there are also new regimens to allow for less intense therapy for patients with lower-risk (mostly node-negative) disease. ${ }^{10}$ But even for these patients, a year of trastuzumab has remained the standard of care after chemotherapy and local therapy (surgery +/- radiation) is finished.

Although trastuzumab significantly improves outcomes for women diagnosed with HER2-positive breast cancer and has few adverse effects (AEs), the disadvantages are that it requires intravenous administration every 3 weeks and can be associated with cardiac AEs. It is also expensive. Because of this, many patients suffer financial toxicity 
from medical bills as well as from time out from work and the need to find extra child or elder care in order to come in every 3 weeks for treatment. In addition, keeping a port for an entire year can be a constant reminder of cancer for patients, which can be challenging emotionally.

Given all of these factors, the question of whether a duration of trastuzumab that is shorter than 1 year may be acceptable for some patients with early-stage HER2-positive breast cancer is an important and very relevant one. Here, we will review the studies that have examined this question and evaluate their results.

\section{Examining the Evidence: 1 Year of Trastuzumab vs a Shorter Duration}

In 2009, the results of the FinHer trial demonstrated that women treated with fluorouracil, epirubicin, and cyclophosphamide (FEC) and docetaxel, and a 9-week course of trastuzumab, had an improvement in distant DFS of $11.7 \%$ at 3 years compared with docetaxel and FEC without trastuzumab (HR, 0.32; $P=.029) .{ }^{11}$ This result was remarkably similar to the joint analysis of the two 2005 studies that showed an improvement in DFS of $11.8 \%$ at 3 years..$^{4-5}$ FinHer (ISRCTN 76560285) was the first trial to show that a shorter duration of treatment might be just as effective as the standard-of-care 12-month duration. Inspired by FinHer's promising results, a number of additional clinical trials were designed to answer this specific question.

The PHARE trial (NCT00381901) was a phase 3, open-label trial in France that randomized 3380 women with early-stage HER2-positive breast cancer to either 6 months or 12 months of adjuvant trastuzumab. The primary outcome was noninferiority of DFS in the 6-month group, with a predefined noninferiority margin of 1.15 . The results showed DFS events in $20.4 \%$ of the 12 -month group compared with $21.2 \%$ in the 6 -month

\section{TABLE 1. Summary of Study Populations for Trials Examining 6 Months vs 1 Year of Trastuzumab}

\begin{tabular}{llll} 
Trial & PHARE & PERSEPHONE & HORG \\
\hline N & 3380 & 4088 & 481 \\
\hline Nodal status & & & 101 \\
\hline Negative & 1842 & 2022 & 204 \\
\hline $\mathbf{1 - 3}$ positive nodes & 1008 & 965 & 176 \\
\hline > 3 positive nodes & 497 & 455 & 0 \\
\hline Missing data & 33 & 620 & 165 \\
\hline Estrogen receptor status & & 1265 & 315 \\
\hline Negative & 1410 & 2823 & 20 \\
\hline Positive & 1970 & 136 & 190 \\
\hline Scarff-Bloom-Richardson grade & 106 & 63 & 252 \\
\hline I & 1351 & 1270 & 8 \\
\hline II & 1843 & 2619 & \\
\hline III & 80 & 136 & \\
\hline Missing data & & & \\
\hline
\end{tabular}

group. The hazard ratio (HR) of 1.08 (95\% CI, 0.93-1.25) included the noninferiority margin, thus the trial did not show noninferiority. However, the overall survival (OS) estimates were similar: The 12-month group had a survival estimate of $86.2 \%$ at 5 years compared with $84.2 \%$ in the 6 -month group. At 7 years, the survival estimate for the 12-month group was $82.3 \%$ compared with $80.6 \%$ for the 6-month group. ${ }^{12}$

The HORG trial ( NCT00712140) was a randomized, controlled noninferiority trial conducted in Greece that also compared 6 months of adjuvant trastuzumab with the standard 12-month duration of therapy in 481 women with early-stage HER2-positive breast cancer. The primary end point was DFS at 3 years. The results demonstrated a 3-year DFS rate of 95.7\% in the 12 -month group and $93.3 \%$ in the 6-month group, representing a difference of $2.4 \%$ and no statistically significant difference in OS (HR, 1.57; 95\% CI, $0.86-2.10 ; P=.137) .{ }^{13}$ Like the PHARE trial, the HORG trial also failed to show noninferiority for the 6-month group; however, both groups still demonstrated very promising 3-year DFS rates.

The PERSEPHONE trial (NCT00712140) took place in the United Kingdom and included 2045 patients with early-stage breast cancer with a noninferiority limit HR set at 1.32. This study demonstrated a 4-year DFS of $89.4 \%$ and $89.8 \%$ in the 6 -month and 12-month groups, respectively, with a HR of 1.07 (90\% CI, 0.93-1.24; $P=$ .011). This study did show noninferiority of 6 months of trastuzumab compared with 12 months. OS at 4 years was also statistically noninferior, at $94.8 \%$ in the 12-month group and $93.8 \%$ in the 6-month group with a HR of $1.14(90 \%$ 


\section{TABLE 2. Summary of Study Populations for Trials Examining 9 Weeks vs 1 Year of Trastuzumab}

\begin{tabular}{lll} 
Trial & SOLD & Short-HER \\
\hline N & 2174 & 1253 \\
\hline Nodal status & & \\
\hline Negative & 1296 & 672 \\
\hline $\mathbf{1 - 3}$ positive nodes & 642 & 383 \\
\hline > 3 positive nodes & 236 & 198 \\
\hline Missing data & 0 & 0 \\
\hline Estrogen receptor status & \\
\hline Negative & 740 & 400 \\
\hline Positive & 1434 & 853 \\
\hline Scarff-Bloom-Richardson grade & \\
\hline I & 857 & 509 \\
\hline II & 1057 & 549 \\
\hline III & 260 & 191 \\
\hline Missing data & 0 & 4 \\
\hline
\end{tabular}

CI, 0.95-1.37; $P=.0010) .{ }^{14}$ The overall numerical results were very similar to those of the PHARE trial, but the slightly larger noninferiority margin between PERSPEHONE and PHARE determined the differences in the final conclusions between the 2 studies. (Table 1)

Two trials examined noninferiority of 9 weeks of trastuzumab compared with 12 months: Short-HER (NCT00629278) and SOLD (NCT00593697). These studies used the promising results of the FinHer trial with 9 weeks of trastuzumab therapy as the experimental group. The Short-HER trial consisted of 1254 patients with early-stage breast cancer and used a noninferiority margin of $\mathrm{HR}$ less than 1.29 . The 5 -year DFS was $88 \%$ in the 12 -month group and $85 \%$ in the 9-week group, with an HR of 1.13 (90\% CI, 0.89-1.42). The 5-year OS was $95.2 \%$ in the 12 -month group and $95.0 \%$ in the 9-week group. ${ }^{15}$ The SOLD trial included 2174 women, and the results did not demonstrate noninferiority, with a 5 -year DFS of $88.0 \%$ in the 9 -week group and of $90.5 \%$ in the 12-month group (HR,1.35; 90\% CI, 1.12-1.72). The 5-year OS did not differ significantly between groups: $94.7 \%$ vs $95.9 \%$ in the 9 -week and 12-month groups, respectively (HR,1.36 90\% CI, 0.98-1.89). ${ }^{16}$ (Table 2)

Of these 5 trials, only PERSPEHONE's results showed noninferiority. In a meta-analysis of the 5 trials, there was a statistically significant difference in the DFS and OS between the shorter duration of treatment-both 9 weeks and 6 months, pooled-compared with the 12-month duration of treatment, with a HR of 1.21 (95\% CI, 1.08-1.37) for DFS and an HR of 1.31 (95\% CI, 1.08-1.59) for OS. However, these differences were not statistically significant for lower-risk patients with node-negative disease (HR, 1.20; $P=.11$ ) and estrogen receptor (ER)-positive disease (HR, 1.15; $P$ = .09). ${ }^{17} \mathrm{~A}$ meta-analysis of the HORG, PERSEPHONE, and PHARE trials more closely examined ER status as an effect modifier on trastuzumab duration. $\mathrm{Pa}$ tients with ER-negative disease had a statistically significant improvement in DFS with the 12-month duration of treatment (HR,1.39; 95\% CI, 1.14-1.70). Patients with ER-positive disease did not have a statistically significant difference in DFS between the 6-month and the 12-month durations of therapy (HR,1.10 95\% CI, 0.90-1.34), indicating that a shorter course of therapy may be acceptable in this patient population. ${ }^{18}$

\section{Risks and Benefits}

Shared decision-making and patient-reported outcomes (PROs) are becoming more important in today's health care system, and especially in breast cancer treatment. A study by Simes et al looked at what patients believed to be an acceptable survival benefit to warrant making the decision to undergo adjuvant chemotherapy for early-stage breast cancer. The authors found that more than $70 \%$ of patients justified treatment for a $5 \%$ or greater improvement in OS at 5 years. Factors that were found to be important in determining patient preferences included support required by patients' dependents, support system, and treatment-related toxicities. ${ }^{19}$ Another study by Tevis et al looked at the benefits and challenges of incorporating PROs into clinical trials. The study recognized that PROs "provide information to support patients in shared decision-making, serve as a measurement of patient-centered care, allow hospitals to monitor outcomes of care over time, play a role in value-based payments, and identify targets for quality improvement efforts." Common metrics to measure PROs include survival and disease control, degree of recovery and functional status, access to treatment, treatment-related 
TABLE 3. Summaries of Trial Results

\begin{tabular}{|c|c|c|c|c|c|c|c|c|c|c|}
\hline Trial & $\mathbf{N}$ & $\begin{array}{l}\text { Primary } \\
\text { end point }\end{array}$ & $\begin{array}{l}\text { Secondary } \\
\text { end point }\end{array}$ & $\begin{array}{l}\text { Duration of } \\
\text { trastuzumab } \\
\text { treatment }\end{array}$ & $\begin{array}{l}\text { Chemotherapy } \\
\text { combination }\end{array}$ & $\begin{array}{l}\text { Median } \\
\text { follow-up } \\
\text { (years) }\end{array}$ & $\begin{array}{l}\text { Predefined } \\
\text { margin of } \\
\text { noninferiority }\end{array}$ & $\begin{array}{l}\text { Survival } \\
\text { HR }(95 \% \\
\text { CI) }\end{array}$ & $\begin{array}{l}\text { Cardiac events } \\
\text { (experimental } \\
\text { vs } 1 \text { yr) }\end{array}$ & $\begin{array}{l}\text { Noninferior } \\
\text { to } 1 \text { year }\end{array}$ \\
\hline PERSEPHONE & 2045 & DFS & $\begin{array}{l}\text { OS, cardiac } \\
\text { events, cost- } \\
\text { effectiveness }\end{array}$ & 6 months & $\begin{array}{l}\text { Anthracycline/ } \\
\text { taxane }+\mathrm{T} \\
\text { (concurrent/ } \\
\text { sequential) }\end{array}$ & 4.9 & $\mathrm{HR}<1.32$ & $\begin{array}{l}\text { DFS: } 1.05 \\
\text { (0.88-1.25); } \\
\text { OS: } 1.14 \\
(0.95-1.37)\end{array}$ & $4.01 \%$ vs $8.02 \%$ & Yes \\
\hline HORG & 481 & DFS & OS, toxicity & 6 months & FEC-D + T & 4.2 & $\begin{array}{l}\text { HR }<1.53 ; 3-y r \\
\text { DFS }\end{array}$ & $\begin{array}{l}\text { DFS: } 1.57 \\
\text { (0.86-2.10); } \\
\text { OS: } 1.45 \\
(0.57-3.67)\end{array}$ & - & No \\
\hline PHARE & 3380 & DFS & $\begin{array}{l}\text { OS, cardiac } \\
\text { events }\end{array}$ & 6 months & $\begin{array}{l}\text { Anthracycline/ } \\
\text { taxane + T } \\
\text { (concurrent/ } \\
\text { sequential) }\end{array}$ & 3.5 & $\begin{array}{l}\text { HR }<1.15 ; 3-y r \\
\text { DFS }\end{array}$ & $\begin{array}{l}\text { DFS: } 1.28 \\
\text { (1.05-1.56); } \\
\text { OS: } 1.46 \\
(1.06-2.01)\end{array}$ & $3.96 \%$ vs $6.57 \%$ & No \\
\hline Short-HER & 1253 & DFS, OS & $\begin{array}{l}\text { Failure rate, } \\
\text { cardiac } \\
\text { events }\end{array}$ & 9 weeks & $\begin{array}{l}\text { AC or EC-D or } \\
\text { FEC-D }+T\end{array}$ & 5.2 & $\begin{array}{l}\mathrm{HR}<1.29 ; 5-y r \\
\text { DFS }\end{array}$ & $\begin{array}{l}\text { DFS: } 1.15 \\
\text { (0.91-1.46); } \\
\text { OS: } 1.06 \\
(0.73-1.55)\end{array}$ & $1.28 \%$ vs $2.87 \%$ & No \\
\hline SOLD & 2174 & DFS & $\begin{array}{l}\text { Cardiac } \\
\text { events }\end{array}$ & 9 weeks & FEC-D + T & 5.2 & $\mathrm{HR}<1.30$ & $\begin{array}{l}\text { DFS: } 1.39 \\
\text { (1.12-1.72); } \\
\text { OS: } 1.36 \\
(0.98-1.89)\end{array}$ & $2.03 \%$ vs $3.86 \%$ & No \\
\hline
\end{tabular}

A, Adriamycin; C, cyclophosphamide; D, docetaxel; DFS, disease-free survival; E, epirubicin; F, fluorouracil; HR, hazard ratio; OS, overall survival; T, trastuzumab.

complications, health-related quality of life, and long-term consequences of therapy. ${ }^{20}$

It is important to consider these PROs and preferences in context of the limited absolute benefit seen with shorter vs longer courses of trastuzumab, particularly for ER-positive, node-negative patients. Additionally, given the recent coronavirus disease 2019 (COVID-19) pandemic, it is important to consider the risk that frequent contact with the health care system poses for patients being treated for breast cancer. Cancer patients are reported to have a high risk of complications and death from COVID-19, ${ }^{21}$ and it is important to minimize their risk of exposure while still providing adequate treatment.

\section{Special Considerations: the COVID-19 Pandemic}

A recent study by Kuderer et al published in The Lancet examined the outcomes of a cohort of cancer patients with COVID-19 across the United States, Canada, and Spain, and found that, compared with patients without cancer who contracted COVID-19, patients with cancer and COVID-19 had a higher associated 30-day all-cause mortality; this was especially true of patients with increased age (odds ratio [OR], 1.84; 95\% CI, 1.52-2.10), $\geq 2$ comorbidities (OR, 4.5; 95\% CI, 1.33-15.28), and active cancer (OR, 5.20; 95\% CI, 2.77-9.77). Breast cancer represented the most common type of malignancy in the study, affecting $21 \%$ of patients. $^{22}$ Another study examining patients hospitalized due to COVID-19 in China's Huebi province compared 105 patients with cancer and 536 patients without cancer; the results indicated that that those with cancer had a higher death rate (OR, 2.34; 95\% CI, 1.15$4.77) .^{23}$

In response to the increased burden on the health care system during the COVID-19 pandemic and the recognition that cancer patients represent a high-risk population, breast cancer organizations implemented guidelines for treatment to minimize the risk of contracting COVID-19. The COVID-19 Breast Cancer Consortium included representatives from the American Society of Breast Surgeons, the National Accreditation Program for Breast Centers, the National Comprehensive Care Network, the Commission on Cancer, and the American College of Radiology, and its recommendations state that it is acceptable to consider reducing the duration of trastuzumab therapy to 6 months due to the "substantial survival benefit and the absence of data suggesting any detrimental defect of use during the pandemic." 24 Similarly, the guidelines released by the European Society for Medical Oncology recommend that "for selected HER2-positive breast cancer, low-risk or elderly patients with cardiovascular or other comorbidities, adjuvant anti-HER2 therapy may reasonably be discontinued after 6 months 


\begin{tabular}{|c|c|c|c|c|c|}
\hline $\begin{array}{l}\text { Dose } \\
(\mathbf{m g} / \mathbf{k g})\end{array}$ & $\begin{array}{l}\text { Dose } \\
(75 \mathrm{~kg})\end{array}$ & $\begin{array}{l}\text { Cost per dose } \\
\text { to payer }\end{array}$ & $\begin{array}{l}\text { Cost per dose to } \\
\text { institution }\end{array}$ & Doses (\#) & $\begin{array}{l}\text { Annual cost } \\
\text { to payer }\end{array}$ \\
\hline 8 & $600 \mathrm{mg}$ & $\$ 13,966$ & $\$ 6950$ & 1 & $\$ 13,966$ \\
\hline \multirow[t]{2}{*}{6} & $450 \mathrm{mg}$ & $\$ 10,467$ & $\$ 5212$ & 16 & $\$ 167,472$ \\
\hline & & & & Total & $\$ 181,438$ \\
\hline
\end{tabular}

instead of 12 months.” ${ }^{25}$ Italy released recommendations that were adopted during the COVID-19 outbreak in a study published in The Oncologist, which discussed the strategies used at the beginning of the outbreak to ensure best possible care while mitigating the risk of infection using a personalized risk-benefit ratio. For patients who were determined to be at "high risk" of infection-of older age and with comorbidities-it was also recommended to consider shortening duration of trastuzumab therapy to 6 months. ${ }^{26}$ In the span of a few weeks, health care systems were challenged to transform how cancer care is delivered in the setting of a pandemic. While it will be essential to resume certain care practices during the recovery phase, there may be closer consideration of changes that were found to be beneficial to patient care, such as reduced duration of trastuzumab therapy.

\section{Cardiotoxicity}

Trastuzumab has been shown to have potential cardiac-related AEs. In all 5 studies included in Table 3, the patients received baseline echocardiograms, and women with preexisting cardiac disease were excluded. Cardiac events in the PHARE and the PERSEPHONE trial were analyzed separately. In the PHARE trial, $5.9 \%$ of patients in the 12 -month group and $3.4 \%$ of patients in the 6-month group experienced New York Heart Association Class I or II car- diac dysfunction during therapy with trastuzumab. ${ }^{27}$ In the PERSEPHONE trial, there was significantly more clinical cardiac toxicity in the 12-month group compared with the 6-month group (13\% vs $10 \% ; P=.04)$, and twice as many 12 -month as 6 -month patients stopped early because of cardiac events ( $8 \%$ vs $4 \% ; P<.0001)$. In the 12 -month group, $8 \%$ of patients had a decrease in left ventricular ejection fraction (LVEF) to $<50 \%$ compared with $6 \%$ in the 6-month group $(P=$ $.004)$, which suggests a longer period of cardiac stress and slower recovery. Age was shown to be an independent risk factor, with patients aged $>70$ years having an OR for cardiac dysfunction of 2.72. Patients with a lower LVEF at baseline (between 50\% and 55\%) had an OR of 5.22 for developing cardiac dysfunction. ${ }^{28}$ In any study, it is important to consider generalizability of the study population. Initial exclusion criteria in the PHARE trial included patients who had previous medical conditions prohibiting the use of trastuzumab, such as documented congestive heart failure, arrythmias, angina pectoris, severe dyspnea at rest, and oxygen dependency. Patients who would have a difficult time with follow-up, and patients who could not have regular controls from social, geographic, or psychological reasons were also excluded. ${ }^{12}$ Many patients with early-stage breast cancer also have 1 or more of these risk factors and/or meet these exclusion criteria, and for them, a shorter duration of treatment of trastuzumab may be more appropriate than the standard duration.

\section{Financial Toxicity}

With the exponentially rising cost of health care in today's practice environment, it is important to consider the cost-effectiveness of treatment when considering 2 different treatment durations. At the time the PERSEPHONE trial was published, the average costs for patients in the 6-month and 12-month arms were 2538.64 British pounds ( US\$3299.4) and 12,333.83 British pounds ( US\$16,033.98), respectively. ${ }^{29}$ Currently in the United States, the cost of treatment is much higher than at the time of the PERSEPHONE trial. The current cost of 12 months of trastuzumab every 3 weeks for a person weighing $75 \mathrm{~kg}$ is shown in Table 4.

This cost information was obtained from average wholesale pricing and will vary by institution; however, it still allows for an estimate of the overall cost of a 12-month duration of therapy. The annual cost to the patient before insurance is $\$ 181,438$ and the annual cost to the institution is $\$ 90,342$. The cost differential is dramatic: an \$83,736 difference between a 12-month and a 6-month duration of treatment. For patients lucky enough to have insurance that covers most of the cost, this is not an issue. For patients who have limited or no insurance, the cost 
of treatment could financially devastate them and their families for decades. Results of a 2018 study published in the American Journal of Medicine found that patients with cancer are 2.65 times more likely to file for bankruptcy than those without cancer, which increases their risk for death. ${ }^{30}$ The rapid advancements in breast cancer therapy often lead to more expensive treatments, higher deductibles, and more co-pays, all of which contribute to financial toxicity and ongoing anxiety around the diagnosis and treatment of breast cancer.

\section{Emotional Costs}

The cost of treatment is not the only financial toxicity affecting patients with early-stage breast cancer. There is also the issue of missing work to undergo treatment. The first infusion of trastuzumab takes around 90 minutes while the subsequent infusions take $30 \mathrm{~min}$ utes, but each treatment requires a visit to the infusion center for treatment, and periodically, a visit with the prescribing physician beforehand is required as well. Transportation time to these appointments and waiting room time also must be factored into the total duration of each trastuzumab treatment, which is all time a patient spends away from family, work, or other activities they enjoy. A prospective study from France looked at the impact of breast cancer treatment on employment for a cohort of 1874 women and found that at 2 years after diagnosis, $21 \%$ of patients had not returned to work. Patients treated with chemotherapy plus trastuzumab had significantly higher odds of not returning to work at 2 years (OR, $2.15 ; 95 \%$ CI, 1.29-3.57) compared with chemotherapy plus hormone ther- apy. Patients treated with chemotherapy alone also had increased odds of not returning to work $(\mathrm{OR}, 1.58 ; 95 \% \mathrm{CI}$, 1.05-2.37); however, the difference was not as significant as for chemotherapy plus trastuzumab. The study authors commented on potential reasons for the statistically significant differences in returning to work; they noted that for patients being treated with trastuzumab, specifically, those reasons can include persistent fatigue, being more likely to perceive themselves as sick for a longer time, and being overwhelmed with accumulation of treatments. ${ }^{31}$

\section{Conclusions}

Results from the individual randomized controlled trials, with the exception of the PERSEPHONE trial, and the pooled meta-analysis of all 5 trials, demonstrate that a treatment duration of 6 months or 9 weeks of trastuzumab is not statistically noninferior to a treatment duration of 12 months. However, for patients with node-negative or ER-positive disease, there was no significant difference between the longer and shorter durations of treatment. For many patients with node-negative and/or ER-positive, HER2-positive breast cancer, a shorter duration of trastuzumab would likely be sufficient, and if they have cardiac or other comorbidities, may even be safer overall. A shorter duration of trastuzum$\mathrm{ab}$ also reduces costs to the patient and the health care system generally, reducing the overall cost of treatment by almost $\$ 100,000$ per patient. Patients who would have a hard time managing a rigorous 12-month trastuzumab treatment schedule, and are in a lower-risk category, would likely have the same benefit from a shorter duration of therapy with far less emotional and financial strain. When the current COVID-19 pandemic is factored in, that pushes the needle even further in the direction of doing less rather than more when appropriate, in order to minimize exposure.

Evidence-based medicine is the backbone of the health care system, but it is critical to consider the difference between statistically significant data and clinically significant data, and to discuss the numbers as well as the realities with our patients. For many patients with early-stage HER2-positive breast cancer, a shorter course of trastuzumab may be more appropriate than a full year of treatment when taking into account financial and logistical considerations as well as their other medical conditions.

FINANCIAL DISCLOSURE: The authors have no significant financial interest in or other relationship with the manufacturer of any product or provider of any service mentioned in this article.

For full reference list, visit cancernetwork.com/trastuzumabreview

\section{cancerNetwork.com} For more great content check out our website.

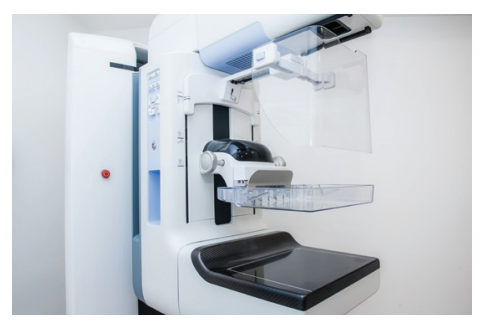

\section{Earlier Breast Screening May Reduce Mortality in Survivors of Childhood Cancer}

cancernetwork.com/early_bc_screening

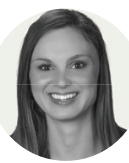

Taylor

Department of Medicine, Emory University School of Medicine.

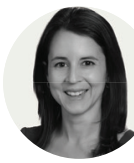

\section{Meisel}

is a medical oncologist at the Winship Cancer Institute and assistant professor in the Department of Hematology and Medical Oncology at Emory University School of Medicine.

\section{Byers}

is a clinical pharmacy specialist in medical oncology at Winship Cancer Institute and Emory University School of Medicine. 


\section{MEET OUR EXPERT}

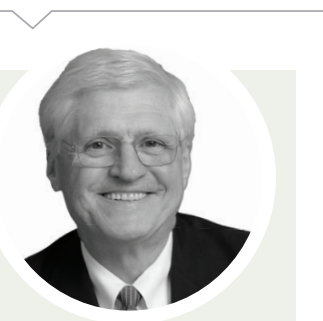

\section{The New Era in Lung Cancer Care}

Treatment options for patients with lung cancer are rapidly improving, leading to better and more durable outcomes. Since

Kris is a medical oncologist at Memorial Sloan Kettering Cancer Center. every patient] about the role for other treatments. For example, radiation in metastatic lung cancer, after some benefit from a systemic therapy, is something that's done routinely now, and something that would never have been done years ago. In fact, if you did radiation, you'd be outed as somebody who was practicing way outside the [standard of] care. But that's now a standard thing. And I think there are good reasons for that, biological and practical as well. So, I think that's among the biggest things - that we now need to consider everything that could be done.

In a case presented at last year's [New York Lung Cancers Symposium] a small tumor in a patient was discovered, and the tumor could be removed surgically, it could be treated with radiation, or intervention radiologists using a bladed procedure could also do it. So how do you choose among these options, particularly for that individual patient? And that's a challenge. It really is. This is the discussion that goes on in an institution-when you say, I'm going to operate on this person, the radiation oncologist says, well, wait a minute, have you considered this? Or the intervention radiologist says that, too, and how do you take all those opinions and choose what the right treatment is?

And now, after you have a curative surgery, who gets additional systemic treatments? I think this year the presentation at virtual ASCO [on results from the phase 3 ADAURA trial], about giving osimertinib to patients with resected lung cancers, is going to change things, because normally many of those patients would not have been considered for therapy, or if they were considered for chemotherapy, would be considered too complicated of a patient. But this is now an option that most patients could have. So, every patient needs to have this consideration of treatment.

Another issue is the need to consider every patient for testing now. It was not a standard of care to do mutation testing on every patient who had a resected lung cancer. It is now. So how are we going to do that? And why are we going to do that? So, these are the kind of things that are important right now, taking advantage of breakthroughs and putting them into practice.

\section{Can you talk a little 1. more about the importance of genomic testing for lung cancer?}

KRIS: There are characteristics of every tumor that define the course of the illness. Understanding those characteristicsand it might be a mutation, it might be amplification, but it also might be what kind of T cells are in that person's tumor-can point you to a therapy. I think EGFR is a very good example. It's very specific. If you have this EGFR mutation, the tumor cells are very sensitive. It's also a diagnostic test for lung cancer. No other cancer has 
these EGFR mutations. If you have it, you've got lung cancer. There are a lot of implications of that. But [having a] better biologic understanding gives you a better chance to match a treatment to the cancer in that person. And we all want to do that. That's something we've all struggled to do, instead of giving treatment ' $X$ ' to everybody. We're doing that again now with checkpoint inhibitors. Everybody gets a checkpoint at some point in their care, unless they have a target. But I think that's the biggest thing about the mutation testing: It helps you define a patient, it helps you define the tumor in a patient and lets you match the treatment. And we're trying to carry that paradigm forward as much as we can.

\section{Dhat are some of the other major changes you've seen over the course of your career?}

KRIS: When I began in this area, there was no treatment for metastatic lung cancer. So, if you were 53 years old and you had lung cancer that spread to your liver, you were sent to the phase 1 clinic, because there was no treatment. And if you were going to go on a National Cancer Institute trial, any drug that they had would be given to you as the first treatment. Any drug. Whether it had any relevance to lung cancer or not, that was the standard of care.

Now, we have a lot of treatments that are specific to lung cancer that really changed the face of the illness. Previously, there was an unmet need; people didn't focus on it. And I could agree with those people because there wasn't yet a clear path forward. A lot of the tough cancers have that characteristic, because there isn't a clear path forward. The investigators and young doctors entering research groups say, "Well, why are you going [into lung cancer]? Stick with leukemia, we've made some progress, and if we understood it better, we'd make even more."

Whereas with lung cancer, there was no progress other than operating. So where are you going to start? That kind of was the issue with me. I was very lucky because I was allied here with our surgeons. Our surgeons were experts and they knew everything about the illness. They knew everything about surgery, including where the problems were-like those cases where they did successful surgery, but the patient didn't survive anyway because the cancer grew back. And it was the surgical leadership, actually, that dragged all of us into trying to do adjuvant and new adjuvant treatments to patients, because they saw that people, despite a successful surgery, had metastasis at some point and they look for systemic therapies. But that was very helpful to me, too, because we had that the surgical base here, and they really focused in on the problem: the people who weren't cured by an operation. They gave us the chance to help cure those people.

\section{To you, what's the 1. most exciting work going on right now in your field?}

KRIS: Well, the bottom line is to cure [the patients]. So, things that have a chance to cure people with advanced lung cancers are, to me, the most exciting, and one of those things would be the use of immunotherapeutics.

When you look at the clinical trial results for people who got immunotherapeutic agents, you see that in some patients with stage IV cancers, the cancer doesn't come back. We all have these patients in our practice, and it's pretty unusual for patients with metastatic cancer for the cancer just not to come back. Their life goes back to normal. It's pretty amazing, and it's kind of interesting that, [if you read the] medical literature, nobody wants to say they're cured. It's the same in other cancers, too, like the melanoma space. People don't want to say these people are cured 5 years later after having metastatic melanoma, and no cancers have come back. Every disease site is controlled, but people don't want to say they're cured.

\section{Which trials or 1.: treatments are making the biggest impact or poised to make the biggest impact in the lung cancer space right now?}

KRIS: I think the data Roy Herbst presented [from the phase 3 ADAURA trial] at the virtual ASCO meeting [represent] a game changer. Going forward, large clinical trials, that are completed already, studied giving postoperative checkpoint inhibitors, T cells, and preoperative checkpoint inhibitors in an attempt to improve outcomes with surgeries or surgery and chemotherapy. We're waiting for the results. So, that's the next very hopeful group of trials that [could] change therapy. The other big completed trial was of durvalumab (Imfinzi) after concurrent chemotherapy and radiation. We went for decades without improving upon giving chemotherapy and radiation for people with locally advanced, but not steady, cancer-people who were not able to have surgery. We tried all kinds of things; nothing helped. In fact, some things harmed. And then suddenly, by giving a year of durvalumab, more people are cancer free afterward and more people are cured there, too. So, I think that's the other really important trial of the last 5 years.

And we continue to chip away, with new drugs for the various mutations: $M E T$, HER2, BRAF. Drugs for all of these are getting approved. Capmatinib (Tabrecta) for MET exon 14, entrectinib (Rozlytrek) for ROS1, these drugs...they chip away at [the cancer] but they're not yet part of a curative package. They shrink the cancer and they shrink it well for a period of time, but it doesn't lead to a cure. But trying to parlay that information to a cure is our challenge.

\section{Q:}

How is coronavirus disease 2019 (COVID-19) affecting care? How has it changed your practice?

KRIS: Well, an addition [to the New York Lung Cancers Symposium] this year will be a session on practice and tele-oncology: how to look for ways to provide care and do it in a way that is appropriate right now with COVID-19. I think the message is, you can't - and we'd like to think we didn't-you can't stop the delivery of care for patients with lung cancers, for any 
reason. It can't be stopped. So our challenge is, How do we continue to provide the same level of care, and the same complexity, with the right decision making, when there's this unbelievable force pervading everything and making it very, very hard to continue care? That was the issue for us. It wasn't taking care of COVID-19. It was continuing care in the face of COVID-19. So, for our patients, with all the different issues that they face, none of them went away. And COVID-19 was added to that list. So, that's the problem, both for us as doctors, and for our patients and their families.

The other thing is, and this was an unintended consequence, we tell people put your care team together, have the people that are important to you be at the visit, be your second set of ears, be there to ask the questions that you might forget. And now that's gone. That person isn't there. They're banned from the building. So, they're physically isolated, and then they're humanly isolated by the people that they have chosen to rely on, to help them make these decisions. So, that's been a real hardship for patients.

FINANCIAL DISCLOSURE: The authors have no significant financial interest in or other relationship with the manufacturer of any product or provider of any service mentioned in this article.

\section{REFERENCE}

1. FDA approvals in lung cancer treatment. Lung Cancer Research Foundation. Updated June 2020. Accessed July 21,2020 . https://www. lungcancerresearchfoundation.org/ research/why-research/treatmentadvances/

\section{CLINICAL TRIALS IN PROGRESS}

\author{
STARTAR TRIAL
}

TITLE : A Salvage Trial of AR Inhibition With ADT and Apalutamide With Radiation Therapy Followed by Docetaxel in Men With PSA Recurrent Prostate Cancer After Radical Prostatectomy (STARTAR; NCT03311555)

BACKGROUND: It was initially shown in the phase 2 STREAM trial (which combined 6 months of androgendeprivation therapy [ADT] with $160 \mathrm{mg} /$ day enzalutamide and salvage radiation to the prostate bed) that patients had 2-year progression-free survival of $65 \%$.

The addition of enzalutamide did not cause any additional toxicities.

Apalutamide is a second-generation androgen receptor (AR) antagonist similar to enzalutamide and would likely have similar activity in this patient population. Based on the CHAARTED study showing benefit of docetaxel in metastatic hormone-sensitive prostate cancer, 6 cycles of docetaxel have been included as part of the regimen to further enhance the efficacy.

Hence, with STARTAR, we aim to further prolong prostate-specific antigen (PSA) recurrence-free survival in men with recurrent disease after radical prostatectomy and high-risk features by combining standard ADT and salvage external beam radiation with the AR inhibitor apalutamide and 6 cycles of docetaxel.

STARTAR is a nonblinded, single-arm phase 2 multicenter study. The end points are 36-month (3-year) PSA recurrence-free survival and the proportion of men at
12, 24, and 36 months with a PSA of less than $0.1 \mathrm{ng} / \mathrm{mL}$ who also have testosterone recovery to more than $100 \mathrm{ng} / \mathrm{dl}$.

STARTAR is an investigator-initiated trial, funded by Janssen; it has opened within the Department of Defense Prostate Cancer Clinical Trials Consortium.

INCLUSION CRITERIA: Eligible men should have prostatectomy; PSA greater than $0.2 \mathrm{ng} / \mathrm{mL}$ and less than $4 \mathrm{ng} / \mathrm{mL}$; and absence of metastatic disease on CT and bone scans. Men should have a prior Gleason score of 8 to 10 ; it may be 7 with at least one of the following: pT3 disease, positive margins, or 4 or fewer positive nodes with adequate laboratory parameters. None of the following are allowed: prior systemic chemotherapy, immunotherapy, or sipuleucel-T (Provenge).

\section{PATIENT ACCRUAL INFORMATION}

- Open date: open to enrollment at Duke in March 2018. Also open in Wake Forest, Cornell, and Nebraska.

- Accrual goal: 42.

- Percent accrued: $93 \%$ enrolled; 3 slots remaining. First patient enrolled in March 2018.

STUDY SITES: Four: Duke Cancer Center(lead site); Wake Forest University Comprehensive Cancer Center; Weill Cornell Medical College, New York; and the GU [genitourinary] Research Network, Omaha, Nebraska. recurrent PSA-only prostate cancer within 4 years of

\section{SCREENING}

\begin{tabular}{|c|c|}
\hline $\begin{array}{c}\text { - prostate adenocarcinoma } \\
\text { - PSA relapse within 4 years } \\
\text { of prostatectomy }\end{array}$ & $\begin{array}{c}\text { E } \\
\text { N } \\
\text { - Detectable and rising PSA } \\
>0.20 \mathrm{ng} / \mathrm{mL}\end{array}$ \\
- Gleason score: $8-10$ or \\
7 with at least one of \\
- pT3 disease \\
or \\
- positive margins \\
or \\
- 4 or fewer positive \\
nodes
\end{tabular}$\quad \begin{gathered}\text { L } \\
\text { L } \\
\text { M } \\
\text { N } \\
\text { T }\end{gathered}$

\section{Co-principal investigators:}

Tian Zhang, MD, MHS

Duke University Medical Center 10386

Durham NC 27710

tian.zhang2@duke.edu

Phone: (919) 668-4667

Fax: (919) 660-0178
Andrew Armstrong, MD, ScM

Duke University Medical Center 103861

Durham NC 27710

andrew.armstrong@duke.edu

Phone: (919) 668-4667

Fax: (919) 660-0178 


\section{Experts Develop New Guideline for Advanced Prostate Cancer}

Jason M. Broderick

$\mathbf{T}$ 2020 (American Urological Association) AUA Virtual Experience included expert insight on the new AUA Guideline for Advanced Prostate Cancer from 2 experts on the panel that created the guideline. ${ }^{1}$

In their discussion during the AUA virtual platform, William Lowrance, $\mathrm{MD}, \mathrm{MPH}$, investigator, Huntsman Cancer Institute and associate professor, Division of Urology, University of Utah in Salt Lake City; and Michael S. Cookson, MD, professor and chairman Department of Urology, the Stephenson Cancer Center and University of Oklahoma College of Medicine in Oklahoma City, highlighted the core components of the consensus guideline for urologists to follow in their daily practice.

"The primary aim of this guideline is to assist clinicians with decision-making. The evidence-based guideline recommendations are furnished according to disease state across the entire continuum of advanced prostate cancer," said Lowrance, who served as chair of the panel.

The focus of the guideline is patients who have exhausted all local therapy options and now require systemic treatment options. These include patients with biochemical recurrence, metastatic hormone-sensitive prostate cancer (mHSPC), nonmetastatic CRPC, and metastatic CRPC. The guideline grades the strength of evidence as high (A), moderate (B), and low or very low (both under the umbrella of $\mathrm{C}$ ).

\section{Early Evaluation and Counseling}

The guideline lays out principles that should be followed with the early evaluation and counseling of patients with suspicion of advanced prostate cancer who do not yet have histologic confirmation. Lowrance said a tissue diagnosis from the primary tumor or metastatic site should be obtained by the clinician.

When diagnosis is confirmed, the guideline recommends physician-patient discussions of treatment options that cover these factors: patients' tumor characteristics, life expectancy, comorbidities, and treatment preferences. The treatment strategy should, if possible, include a multidisciplinary approach. Also, when possible, pain control and other symptom support should be optimized, explained Lowrance.

\section{Biochemical Recurrence Without Metastatic Disease}

In the hormone-sensitive setting, PSA recurrence almost always precedes clinical detection of metastases," said Lowrance. Thus, in men with biochemical recurrence without metastases, clinicians should perform serial PSA measurements, clinical evaluations, and staging evaluations.

For these staging evaluations, the guideline recommends use of standard- of-care imaging, comprising bone scans and axial imaging with CT or MRI Given the emergence of advanced PET imaging in the paradigm for advanced prostate cancer, the guideline stipulates that, "Clinicians may utilize novel PETCT scans in patients with PSA recurrence after failure of local therapy as an alternative to conventional imaging or in the setting of negative conventional imaging."

In those patients with a rising PSA whose imaging does not reveal any metastases, the guideline recommends that the patients be offered observation or enrollment on a clinical trial. As routine practice, androgen deprivation therapy (ADT) should not be started in these patients.

\section{mHSPC}

The guideline states that genetic counseling and germline testing should be offered to all men with mHSPC, regardless of age and family history. The germline testing in this setting focuses on genes that influence homologous recombination repair, such as BRCA1, BRCA2, and ATM.

Patients with mHSPC should be offered surgical castration or ADT combined with either LHRH agonists or antagonists (grade B). Further, grade A evidence supports the guideline recommendation that "clinicians should offer continued ADT in combination with 
either androgen pathway-directed therapy (eg, abiraterone acetate [Zytiga] plus prednisone; apalutamide [Erleada]; or enzalutamide [Xtandi]) or docetaxelbased chemotherapy," said Lowrance.

Lowrance, shared some of the data supporting the use of androgen pathway-directed therapies in this setting. In the phase 3 TITAN trial $(\mathrm{N}=1050)$, apalutamide plus ADT reduced the risk of death by $33 \%$ versus ADT alone in patients with mHSPC (HR, 0.67; 95\% CI, 0.51-0.89; $P=.005) .{ }^{2}$ And the phase 3 ENZAMET trial $(\mathrm{N}=1125)$, showed the same $33 \%$ reduction in the risk of death with enzalutamide plus ADT versus ADT alone in patients with $\mathrm{mHSPC}$ (HR, 0.67; 95\% 0.52-0.86; $P=.002){ }^{3}$

\section{Nonmetastatic CRPC}

This population comprises patients with a rising PSA but no sign of metastatic disease on standard imaging despite "the persistence of a castrate-level testosterone," said Cookson.

In recent years, the first agents have been specifically approved by the FDA for the treatment of these patients, based on a demonstrated metastasis-free survival (MFS) benefit. All 3 therapies are androgen receptor antagonists: apalutamide, darolutamide (Nubeqa), and enzalutamide.

Apalutamide, enzalutamide, and darolutamide all demonstrated an MFS benefit over placebo in the pivotal SPARTAN (HR, 0.28; $P<.001),{ }^{4}$ PROSPER (HR, 0.29; $P<.001$ ), ${ }^{5}$ and ARAMIS (HR, $0.41 ; P<.001)^{6}$ trials, respectively. All 3 trials enrolled over 1200 patients with nonmetastatic CRPC at high risk for developing metastatic disease (PSA doubling time of $\leq 10$ months). Cookson noted that since the completion of the data analysis for the guideline, overall survival (OS) data from the PROSPER trial were published showing that enzalutamide led to a $27 \%$ reduced risk of death versus placebo, with a median OS of 67 versus 56.3 months, respectively (HR, 0.73; 95\% CI, 0.61-0.89; $P=.001)$.

\section{MCRPC}

Docetaxel is indicated for the first-line treatment of patients with mCRPC with several treatments approved for use before and after the chemotherapy. The immunotherapy sipuleucel-T (Provenge) is approved by the FDA for asymptomatic or minimally symptomatic mCRPC. Enzalutamide and abiraterone acetate (plus prednisone) are approved for use both in chemotherapy-naive patients and following failure of docetaxel. The chemotherapy cabazitaxel (Jevtana) is approved as a second-line, post-docetaxel option.

The radiopharmaceutical radium-223 (Xofigo) is approved for the treatment of patients with CRPC with symptomatic bone metastases but no known visceral metastatic disease. In this setting, radium-223 has demonstrated an OS benefit over standard therapy in both chemotherapy-naïve patients and those with prior docetaxel.

Genetic testing and related treatments are a burgeoning area of the $\mathrm{MCRPC}$ paradigm as research has shown that, "DNA damage response (DDR) alterations have been identified in $23 \%$ of patients with mCRPC, including both somatic and germline defects. Among the DDR alterations, $B C R A 2$ is the most frequently altered, and these gene alterations are expected to confer sensitivity to PARP inhibitors; thus, nearly $20 \%$ of patients with mCRPC may potentially benefit from this therapy," explained Cookson.

The AUA guideline includes the grade $\mathrm{C}$ recommendation that, "Clinicians should offer a PARP inhibitor to patients with deleterious or suspected deleterious germline or somatic homologous recombination repair gene-mutated mCRPC following prior treatment with enzalutamide or abiraterone acetate, and/or a taxane-based chemotherapy. Platinum-based chemotherapy may be offered as an alternative for patients who cannot use or obtain a PARP inhibitor.”

Two PARP inhibitors were recently approved by the FDA for the treatment of patients with mCRPC. In May 2020, the FDA approved rucaparib (Rubraca) for the treatment of adult patients with $B R C A$ mutation (germline and/or somatic)-associated mCRPC who have been treated with androgen receptor-directed therapy and a taxane-based chemotherapy. The approval was based on data from the phase 2 TRITON2 study, in which the confirmed objective response rate was $44 \%$ in a cohort of 62 patients with BRCA-mutated mCRPC. ${ }^{8}$

Also in May 2020, the FDA approved olaparib (Lynparza) for the treatment of adult patients with deleterious or suspected deleterious germline or somatic homologous recombination repair gene-mutated mCRPC who have progressed following prior treatment with enzalutamide or abiraterone acetate. The approval was supported by the phase 3 PROfound trial, in which the risk of disease progression or death was reduced by $66 \%$ with olaparib compared with abiraterone acetate or enzalutamide (HR, 0.34; $P<.0001)$ in patients with BRCA1/2- or ATM-mutant mCRPC. ${ }^{9}$

The final option for mCRPC that Cookson covered was the immune checkpoint inhibitor pembrolizumab, which is available as an option for patients with microsatellite instability-high or mismatch repair-deficient tumors.

\section{Bone Health}

Cookson concluded the discussion with a focus on bone health. Preventative treatments with bisphosphonates or denosumab should be recommended to patients with advanced prostate cancer whose bone loss has put them at high fracture risk, according to the guideline. It is also indicated that, when appropriate, these patients should be referred to experts in the area of osteoporosis. Among patients with $\mathrm{mCRPC}$ with bony metastases, the guidelines include the grade B recommendation that, "Clinicians should prescribe a bone protective agent, denosumab or zoledronic acid, to prevent skeletal-related events," said Cookson.

For full reference list, visit cancernetwork.com/AUA_guideline 


\title{
Locally Advanced Gastrointestinal Stromal Tumor in a 33-Year-Old Woman Seeking to Conceive
}

Mehmet Sitki Copur, MD; Allison M. Cushman-Vokoun, MD, PhD; Abigail Delaney, MD; James Padussis, MD; Whitney Wedel, MD; Scott Lauer, MD; and Geoffrey Talmon, MD

\begin{abstract}
Gastrointestinal stromal tumors (GISTs) are rare neoplasms of the gastrointestinal tract. They commonly present with nonspecific symptoms and thus are often discovered incidentally. They are best identified by CT scan, and most stain positive for CD117 (C-Kit), CD34, and/or DOG-1. Several risk stratification classification systems have been developed based on tumor size, mitotic rate, location, and perforation. Traditional chemotherapy and radiation therapy have been very ineffective, making surgery the mainstay of treatment. The discovery of mutations associated with these tumors has revolutionized the treatment approach. Imatinib mesylate, a selective tyrosine kinase receptor inhibitor, used as adjuvant or neoadjuvant therapy, has greatly improved the morbidity and mortality associated with GISTs. As the survival of patients has increased with the long-term use of targeted therapies, quality-of-life issues now have become much more relevant and have come to the forefront of care. We present a young woman who was successfully treated for GIST but now faces associated long-term adverse effects of imatinib, including the challenge of preserving fertility and the potential for childbearing.
\end{abstract}

\section{Case}

A 33-year-old White woman had been having some nonspecific intermittent abdominal pain for the past 2 years, which had been attributed to various different conditions including uterine fibroids, irritable bowel syndrome, fibromyalgia, and pelvic floor dysfunction. After visits to several different health care provider, a CT scan of the abdomen and pelvis eventually revealed a large lobulated mesenteric mass measuring $13.5 \times 9.5$ $\mathrm{cm}$ (Figure 1). A CT-guided biopsy of this mass revealed proliferation of epithelioid to spindled cells with moderate eosinophilic cytoplasm (Figures 2A and 2B). Immunohistochemical stains of the biopsy specimen were strongly positive for DOG1 (Figure 2C), CKIT, and CAM5.2; weakly positive for cytokeratin AE1/AE3; and negative for CK7, CK20, S100 protein, desmin, chromogranin, synaptophysin, CDX2, TTF1, PAX8, calretinin, and ER. WT1 was positive with cytoplasmic staining but negative with nuclear staining. The mitotic rate was estimated to be at least 2 per 50 high-power fields (HPF). No necrosis was identified.

A gastrointestinal stromal tumor
(GIST) mutation panel (analyzing KIT, $P D G F R \alpha$, and BRAF genes) was performed on DNA extracted from the biopsy using next-generation sequencing ( $50 \%$ estimated tumor cellularity). Two mutations were detected: KIT p.A502 Y503dup (c.1504_1509dupGCCTAT; exon 9; pathogenic; $48 \%$ variant allele frequency) and $B R A F$ p.R444W (c.1330C > T; exon 11; likely pathogenic; $28 \%$ variant allele frequency). No PDGFR $\alpha$ mutations were identified.

After 8 weeks of neoadjuvant therapy with imatinib $800 \mathrm{mg}$ by mouth (po) daily, CT scans showed interval decrease in the patient's mesenteric mass, down to $8.3 \times 6.2 \mathrm{~cm}$ (Figure 3A). After another 4 weeks of neoadjuvant imatinib, CT scans did not show any further significant decrease in the size of the mass ( 8.1 $\mathrm{x} 6.2 \mathrm{~cm}$ ) (Figure 3B).

She underwent exploratory laparotomy with omentectomy, appendectomy excision of mesenteric implants, small bowel resection, and uterine fibroid resection. Pathology of postoperative material showed a jejunal GIST tumor with central hyalinization and calcification, consistent with therapy effect. Greatest tumor dimension was $9.5 \mathrm{~cm}$, mitotic rate was 0 mitoses $/ 5 \mathrm{~mm}^{2}$, and all margins were negative with no nodal involvement. Metastatic GIST was identified in omental nodules and colon-as- 


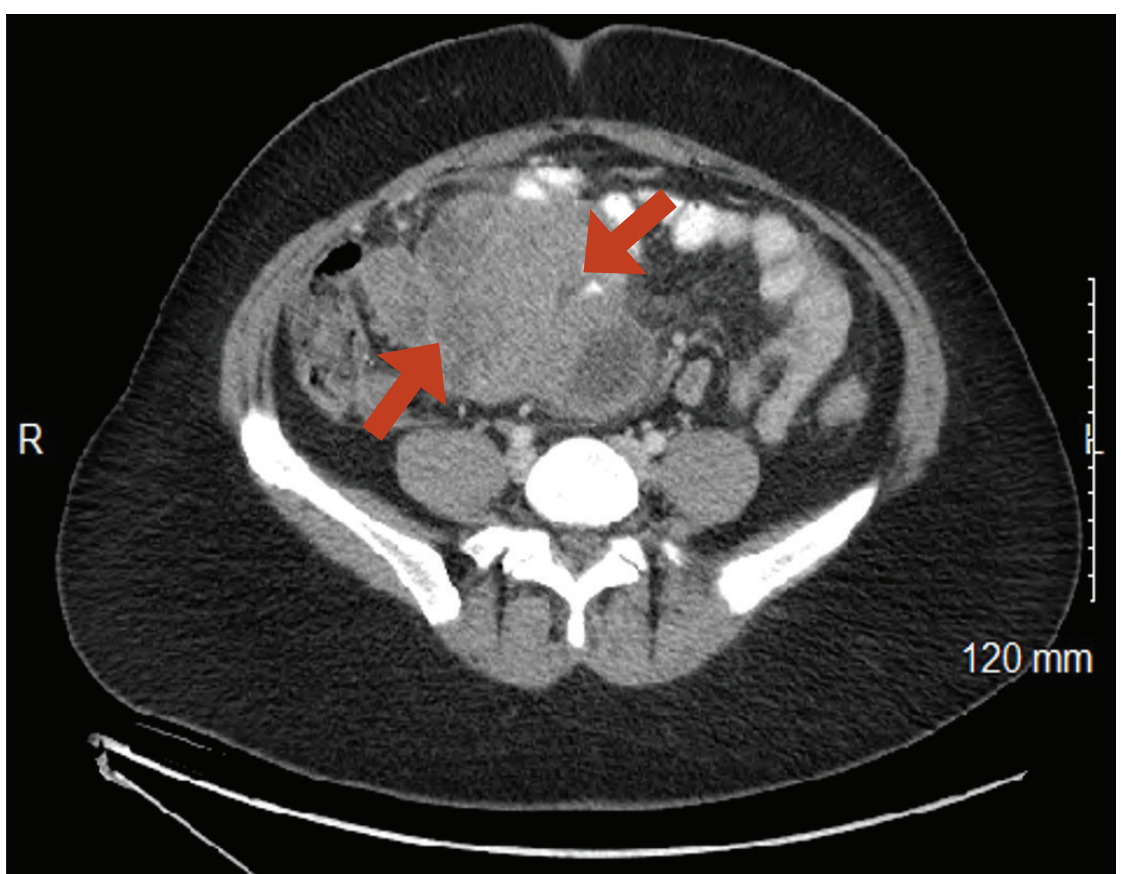

- Figure 1. Lobulated Mesenteric Mass Prior to Neoadjuvant Imatinib.

sociated mesenteric nodules. She was staged as pathologic stage pyT3pyN0pyM1.

After surgery, she was placed on adjuvant imatinib $800 \mathrm{mg}$ daily to be given for the next 3 to 5 years. Her most recent CT scan, done 3 months post surgery, showed no evidence of disease

(Figure 3C). The patient expresses her strong desire to be able to conceive and have her own biologic children in the near future.

\section{Introduction}

GISTs account for less than $1 \%$ of all gastrointestinal neoplasms, yet they constitute the most common soft-tissue sarcomas of the gastrointestinal tract. ${ }^{1}$ While the age-adjusted yearly incidence has been estimated at around 6.8 cases per million, based on a study utilizing Surveillance Epidemiology End Results data, ${ }^{2}$ the actual incidence is not known. This is partly due to lack of universal comprehensive testing for the characteristic KIT and/or PDGFR $\alpha$ gene mutations. Additionally, small, indolent
GIST tumors-each only a few millimeters in diameter-do commonly occur in the general population, but they do not get included in cancer registries. ${ }^{3,4}$ The most common site for GIST tumors is the stomach $(60 \%)$, followed by nonduodenal small intestine (30\%), duodenum $(5 \%)$, and rectum $(4 \%)$; they appear much less commonly in the esophagus, colon, and appendix $(1 \%) .{ }^{5}$ GISTs are equally distributed across all geographic and ethnic groups. Men and women are equally affected. Most patients present aged between 50 and 80 years. ${ }^{6}$

GISTs seem to be associated with no specific symptoms. Patients with a suspected GIST can present with a variety of symptoms, ranging from early satiety to abdominal pain, to gastrointestinal or intraperitoneal bleed with accompanying symptoms of fatigue and anemia. Neoplastic GIST cells appear to originate from a precursor cell lineage in the myenteric plexus of gastrointestinal tract that differentiates into the interstitial cells of Cajal (ICCs). ${ }^{7}$ Most tumors express the characteristic CD117 anti- gen, part of a tyrosine kinase receptor, which has been exploited for the development of novel therapeutic tyrosine kinase inhibitors (TKIs) (Figure 3).

\section{Pathology}

Biopsy is the mainstay of diagnosis. Endoscopic ultrasound-guided biopsies are preferred due to reduced possibility of bleeding and of intra-abdominal tumor spread risk. In advanced cases, image-guided percutaneous biopsies are appropriate. From incidentally discovered benign nodules that measure less than $10 \mathrm{~mm}$ to large sarcomas, GISTs represent a morphological and biological continuum. Despite clinicopathological differences, most GISTs share a similar genetic profile with KIT or PDGFR $\alpha$ gain-of-function mutations (oncogenic mutations). ${ }^{8,9}$ KIT (CD117) is expressed in more than $95 \%$ of GISTs; $80 \%$ of these patients have an activating mutation in the gene encoding for the KIT receptor tyrosine kinase, and in $5 \%$ to $10 \%$ the mutation is in the gene encoding the PDGFR $\alpha$ receptor tyrosine kinase. The remaining $10 \%$ to $15 \%$ of GISTs are KIT and PDGFR $\alpha$ wild-type, and in $10 \%$ to $15 \%$ of these tumors, BRAF p.V600E mutations can occur $\left(<5 \%\right.$ of GISTs overall). ${ }^{10-13}$ KIT mutation in GIST does not cause KIT expression-a fact that is occasionally misunderstood-but it modifies KIT function. ${ }^{5}$ Activation of KIT and PDGFR $\alpha$ by their ligands regulates important cell functions, including proliferation, apoptosis, chemotaxis, and adhesion. KIT function is also critical for the development and maintenance of different cell types, including hematopoietic cells, mast cells, melanocytes, gametocytes, and ICCs. ${ }^{14-16}$ Based on immunophenotypic, ultrastructural, and cell signaling similarities, GISTs are believed to originate from KIT-expressing progenitor ICCs through somatic KIT or PDGFR $\alpha$ mutations, which are believed to be an early step in GIST pathogenesis.

KIT and PDGFR $\alpha$ mutations can be 

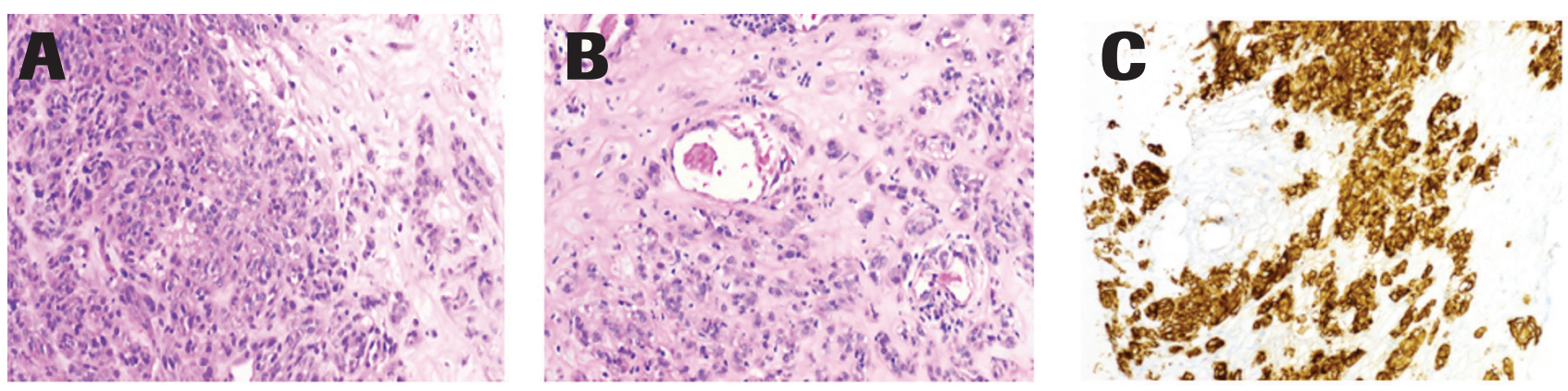

- Figure 2. Sections Demonstrate a Proliferation of Spindled to Epithelioid Cells (hematoxylin and eosin stain, 200x magnification) (A\&B); DOG-1 Immunohistochemical Staining (C).

categorized into 2 groups: mutations in the receptor regulatory domains (extracellular and juxtamembrane) and mutations in the tyrosine kinase enzymatic domains (TK1 and TK2). The majority of KIT mutations occur in the juxtamebrane domain (KIT exon 11), followed by mutations in the extracellular domain (KIT exon 9). Most of the PDGFR $\alpha$ mutations occur in exon 18 in TK2. While exon 11 mutations are common in all sites of GISTs, exon 9 mutations are more likely to be seen in intestinal sites. PDGFR $\alpha$ exon 18 mutations occur more commonly in gastric GISTs. Continuous ligand-independent activation of KIT or PDGFR $\alpha$ kinases through these mutations leads to activation of downstream signal transduction pathways promoting cell survival and proliferation. BRAF is a serine-threonine kinase that functions downstream of receptor tyrosine kinases in the MAPK pathway, to activate cell proliferation. ${ }^{13}$

Immunohistochemical staining of biopsy specimens for CD117, DOG1, and/or CD34, and molecular genetic testing to identify KIT and/or PDGFR $\alpha$ mutations, help establish the diagnosis. Absence of KIT and/or PDGFR $\alpha$ mutations does not exclude the diagnosis of GIST; conversely, KIT positivity alone may not be sufficient to confirm the diagnosis. Tumors lacking KIT, PDGFR $\alpha$, or BRAF mutations should be further evaluated with succinate dehydrogenase immunostaining and, if deficient, germline mutation testing should be considered.

\section{Prognostic Factors}

Tumor size (ie, $>10 \mathrm{~cm}$ ) and mitotic rate (ie, $>5$ mitoses per $5 \mathrm{~mm}^{2}$ ) have been the most widely used pathologic features to predict poor prognosis. Tumor site has been utilized as another important factor for risk stratification; a gastric location indicates less aggressive behavior compared with GIST tumors of intestinal or rectal origin. ${ }^{17,18}$ Tumor genotype is another independent prognostic factor, with KIT exon 11 deletions predicting higher risk of relapse after resection, but also excellent sensitivity to imatinib. ${ }^{19}$ Tumors with KIT exon 9 mutations may have better relapse-free survival (RFS) after curative resection, but they are less sensitive to imatinib therapy (requiring higher doses or alternative TKIs).19 PDGFR $\alpha$ exon 18 mutations have been associated with better prognosis; however, the most common mutation in exon 18 (p.D842V) predicts resistance to early-generation TKIs. ${ }^{18,20}$ Female sex has been implicated as an independent prognostic factor for better progression-free survival (PFS) and overall survival (OS). ${ }^{19}$

\section{Neoadjuvant Therapy}

Between $15 \%$ to $50 \%$ of GIST patients present at an advanced stage at the time of diagnosis. ${ }^{21}$ Prior to the advent of imatinib, the lack of effective systemic treatment options meant surgery was the only recognized treatment for GIST. Notoriously dismal response rates of GISTs to conventional chemotherapy or radiation therapy (overall response rate,
$<5 \%$ ) led to often-poor surgical outcomes in locally advanced disease, with an OS of 12 to 19 months and a 5 -year survival rate of less than $5 \%$ to $10 \% .^{22,23}$ In patients with advanced GISTs, preoperative therapy with imatinib can significantly improve these outcomes in terms of both PFS and fewer intraoperative tumor ruptures and multivisceral resections. ${ }^{24}$ In a prospective study of potentially resectable GIST patients, the efficacy of preoperative $600 \mathrm{mg}$ daily imatinib provided partial response in $7 \%$ and stable disease in $83 \%$ of the patients. The estimated 2-year PFS and OS rates were $83 \%$ and $93 \%$, respectively. ${ }^{25}$ In another prospective study, preoperative imatinib improved resectability and reduced surgical morbidity in patients with primary resectable or unresectable GIST, providing a median tumor size reduction of $34 \%$ and an estimated 3-year PFS rate of $77 \% .{ }^{26}$ While the efficacy and safety of preoperative imatinib has been well established, it is difficult to determine a definitive survival benefit of this approach since all patients have also been treated with postoperative imatinib for at least 2 years. One disadvantage of this approach is that the preoperative imatinib use can impede accurate assessment of recurrence risk. Thus, this approach should be considered only if surgical morbidity would be reduced by downstaging tumor size, as in the case presented here.

\section{Surgery}

Primary treatment of localized or resectable GISTs is surgical resection. 


\section{Abigail Delaney, MD}

\section{Fertility in the Setting of GISTs}

\begin{abstract}
$\triangle$ pproximately 1.7 million people are diagnosed with cancer yearly in the United States and of those, almost $25 \%$ are aged less than 45 years. ${ }^{1}$ Most data estimate that annually, 54,000 Americans treated for cancer will be at risk for infertility. ${ }^{2}$ While much of this is iatrogenic, in that many chemotherapeutic drugs and radiation treatments are gonadotoxic, sometimes infertility results simply as a delay in the ability to pursue childbearing, secondary to cancer treatment. This is particularly true with regard to female patients, as a woman's reproductive lifespan is finite and begins to decline exponentially beyond age 37 . When cancer patients and their family members are surveyed post treatment, they consistently express regret both at not being presented with fertility preservation options and at the general lack of counseling with respect to how their treatment would affect future childbearing.

The authors in this publication describe the case of a young woman diagnosed with a gastrointestinal stromal tumor (GIST) at age 33. As the treatment of GISTs has improved, particularly with advent of imatinib, focus on quality of life after cancer has
\end{abstract}

Perioperative imatinib has been utilized to decrease surgical morbidity prior to surgery or if residual tumor remains after surgery. GISTs are friable tumors with a propensity to rupture. The principal goal of surgery is to achieve complete resection with clear margins and an intact pseudocapsule. In the absence of pathologically enlarged nodes, lymphadenectomy is not necessary because of the low incidence of nodal involvement. For microscopically positive margins, re-resection is not required. If a complex surgical procedure become paramount. In young women like this patient, so-called quality of life after cancer often includes family planning and childbearing. While this woman was young at diagnosis, adjuvant therapy with imatinib may be maintained for 3 to 5 years, and so she will be of advanced maternal age (36 to 38 years) by the time she has completed her treatment. Simply by delaying her childbearing, she will compromise her fertility due to age-related diminishing ovarian reserve. Many patients desire fertility preservation with either oocyte or embryo cryopreservation, knowing that without such a procedure, this delay could render them unable to complete their family plan.

Literature regarding imatinib treatment and future fertility is scarce. Most data regarding imatinib and fertility involve the scenario of unplanned pregnancy during active treatment. $^{3}$ Although the majority of data indicate that pregnancy clearly is possible while taking imatinib or after treatment, longterm studies of reproductive capacity are limited. A single case report describes diminished ovarian response at time of oocyte retrieval in an adolescent fertility preservation case. Egg quality and ovarian response was dramatically improved after 2 months off the medication. ${ }^{4}$ More studies and long-term data are necessary so patients can be adequately counseled regarding future fertility after treatment.

The future for young cancer patients desiring fertility after treatment remains bright. Continued improvements in assisted reproductive technology have allowed thousands of patients to achieve their childbearing potential after cancer treatments. With improved dialogue, teamwork, and education, we can continue to provide patients with the best quality of life after cancer.

\section{REFERENCES}

1. National Cancer Institute, Surveillance, Epidemiology, and End Results Program. U.S. population data: 1969-2018. December 2019. Accessed June 8, 2020. https://www.seer. cancer.gov/popdata

2. Woodruff TK. Oncofertility: a grand collaboration between reproductive medicine and oncology. Reproduction. 2015;150(3):S1S10. doi:10.1530/REP-15-0163

3. Pye SM, Cortes J, Ault P, et al. The effects of imatinib on pregnancy outcome. Blood. 2008;111(12):5505-5508. doi:10.1182/ blood-2007-10-114900

4. Zamah AM, Mauro MJ, Druker BJ, et al. Will imatinib compromise reproductive capacity? Oncologist. 2011;16(10):1422-1427. doi:10.1634/theoncologist.2011-0137 will be needed due to extent of disease, a multidisciplinary tumor board discussion must be held regarding the use of neoadjuvant imatinib. Several case studies have shown the value of preoperative imatinib in enabling organ- and/or sphincter-sparing surgeries and in improving surgical outcomes. $^{27}$

The role of laparoscopy in GIST is an expanding area of research. Despite the lack of prospective studies, some small case series and retrospective studies have revealed the feasibility and benefits of this approach with shorter hospital stays, decreased morbidity, and low recurrence rates. ${ }^{28,29}$ This approach has been most suitable for GISTs measuring less than 5 $\mathrm{cm}$ at favorable anatomic sites, such as the anterior wall of the stomach, jejunum, and ileum, and some small rectal locations.

\section{Adjuvant Therapy}

In approximately $85 \%$ of patients with GIST, complete resection is possible; however, surgery is not always curative. Following complete resection, almost half 

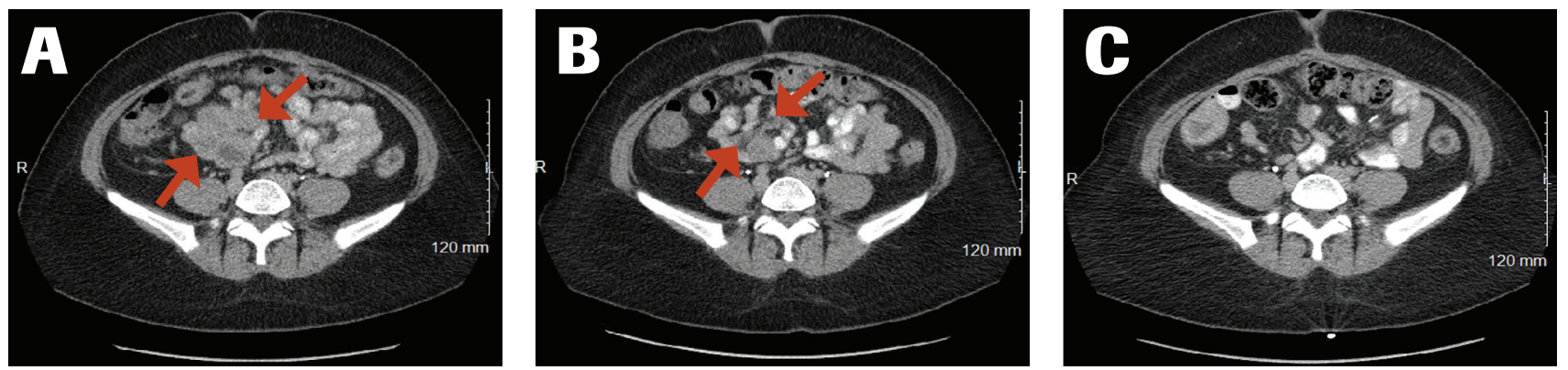

- Figure 3. Interval Decrease of Mesenteric Mass after 8 Weeks of Neoadjuvant Imatinib (A); Neoadjuvant Imatinib (B); Three Months Post Surgery, Showing No Abdominal Disease (C)

of patients develop recurrence or metastatic disease, with a 5-year survival rate of $50 \% .^{30,31}$ Adjuvant imatinib therapy was first evaluated in ACOSOG Z9001, in which patients with $3 \mathrm{~cm}$ or larger primary localized GISTs were randomized to $400 \mathrm{mg}$ daily imatinib or to placebo after complete resection of their tumors for a duration of 1 year. ${ }^{32}$ At a median follow-up of 74 months, RFS was significantly better in the imatinib arm; however, the OS rates were not different between the arms. ${ }^{33}$ Mutational analysis of all patients further revealed that while patients with KIT exon 11 deletions had a higher likelihood of RFS, patients with KIT exon 11 insertion or point mutation, KIT exon 9 mutation, PDGFR $\alpha$ mutation, or wild-type GIST did not. Tumor genotype did not affect RFS in the placebo arm. After the initial ACOSG Z9001, several other randomized studies investigated the optimal duration of adjuvant therapy. The EORTC-62024 study compared 2 years of adjuvant imatinib therapy with observation in patients with localized intermediate-risk or high-risk GISTs. ${ }^{34}$ While the RFS rate was significantly higher in the imatinib arm, the difference between arms in 5-year RFS did not reach statistical significance. In another study, the Scandinavian Sarcoma Group randomized high-risk GIST patients to 12 months vs 36 months of imatinib after curative intent surgery. ${ }^{35}$ This study was the first to show an OS advantage in addition to a RFS advantage. After a median follow-up of 90 months, 5 -year RFS was $71.1 \%$ vs $52.3 \%$, and 5-year OS was $91.9 \%$ vs $85.3 \%$, both in favor of 36-month adjuvant imatinib. Nongastric GIST and high-mitotic count GIST patients were at a higher risk for recurrence. The most recent analysis of this trial (SSGXVIII/AIO) was presented at the American Society of Clinical Oncology 2020 Virtual Meeting. Over a median follow-up of 119 months, $53 \%$ of 1 -year treatment patients experienced a recurrence event, $30 \%$ of those patients died, of whom $82 \%$ died as a result of metastatic GIST. In the 3-year treatment group, 44\% experienced a recurrence event, and $18 \%$ of those patients died, of whom $83 \%$ died as a result of metastatic GIST. OS at 10 years was higher with longer imatinib treatment, at $79 \%$ with 3 years of therapy vs $65 \%$ with 1 year (HR, 0.55 ; $P=.004) .{ }^{36}$ Finally, a single-arm phase 2 trial enrolled high-risk GIST patients onto $400 \mathrm{mg}$ daily imatinib for 5 years or until progression, relapse, or intolerance. ${ }^{37}$ The primary end point was RFS. The 5 - and 8-year estimated RFS rates were 90\% and $81 \%$, respectively, while the 5 - and 8 -year OS rates were both $95 \%$. Nearly half of the patients discontinued treatment early, and most recurrences occurred after treatment discontinuation. Common reasons for early discontinuation included patient choice $(20 \%)$, adverse effects (AEs) $(17 \%)$, protocol deviation (4\%), and loss of follow-up (4\%). The most common AEs of all grades were nausea $(71 \%)$, diarrhea $(63 \%)$, fatigue $(50 \%)$, muscle spasm (41\%), vomiting (39\%), and periorbital edema (34\%).

\section{Adverse Effects}

The most commonly reported AEs of imatinib are fluid retention, diarrhea, nausea, fatigue, muscle cramps, abdominal pain, and rash. All improve with prolonged therapy. ${ }^{38}$ Liver function abnormalities, low blood counts, gastrointestinal bleeding, lung toxicity, and congestive heart failure rarely have been reported. ${ }^{39}$

\section{Fertility}

Modern social structure and medical advances have led to increasing numbers of women choosing childbearing at an older age. Increasing incidence of GISTs and its successful treatment now have brought the issues of preservation of fertility and childbearing potential in younger patients affected with this disease. Although contraception advice and termination of early pregnancy have been the standard practice in patients with this disease, cases of GIST during pregnancy have been reported. ${ }^{40}$

Tan et al presented a meta-analysis ( $\mathrm{N}$ = 12) of women diagnosed with a GIST tumor in the perigestational period. Analysis of these cases demonstrated that 8 of 12 went on to deliver healthy infants. The majority ( 7 of 8 ) of these women were not initiated on imatinib therapy until they reached postpartum status. The study does report a single case of a woman who remained on imatinib therapy for a known GIST tumor throughout the pregnancy. She underwent a successful delivery and the infant had continued normal development up to 1 year later. ${ }^{41}$ 
Limited data are available regarding gestational exposure to imatinib; they consist mostly of case studies and series. Animal data indicate an increased risk of teratogenicity during organogenesis and an increased risk of fetal loss with exposure at equivalent recommended dosages. ${ }^{42,43}$

The largest data set of women found to be gestationally exposed to imatinib $(\mathrm{N}=$ 180) demonstrates potential risk of fetal abnormalities and fetal loss. The majority of exposures in the data set occurred in the first trimester; of these women, $28 \%$ underwent elective termination secondary to treatment. Three of these terminations were performed following identification of multisystem fetal abnormalities on obstetric ultrasound. These study results cited a $14.4 \%$ risk of spontaneous abortion; however, they also demonstrated that normal infants were delivered in $50 \%$ of exposed pregnancies. Given limited evidence of fetal harm, best practices still include prevention of pregnancy while taking imatinib and consideration of termination should a pregnancy occur while taking the drug. ${ }^{44}$

While there are a paucity of data with regard to imatinib in pregnancy, data are even more scarce with regard to fertility while on imatinib therapy or fertility after cessation of therapy. Available literature demonstrates a single case report indicating that active treatment with imatinib can result in reversible diminished ovarian function when undergoing fertility preservation. Long-term data, however, are clearly lacking. ${ }^{45}$

\section{Conclusions}

GISTs are rare tumors that account for a small percentage of gastrointestinal neoplasms and they are usually discovered incidentally. While surgery remains the backbone of treatment, our understanding of aberrant molecular pathways in the pathogenesis of this disease and the rapid development of molecular therapies targeting these pathways have resulted in dramatic results redefining the management of these traditionally chemotherapy-resistant tumors. Neoadjuvant imatinib therapy—with the goal of shrinking the tumor and allowing organ preservation and less extensive surgery than would otherwise be required for some patients—and long-term adjuvant therapy-for patients with high risk of recurrence-now enable many GIST patients to live long, normal lives. Quality of life has now become an important issue for patients on long-term TKI therapy.

\section{Outcome of the Case}

After successful curative-intent surgery, the patient remains on 800-mg adjuvant imatinib with a goal of 3 to 5 years of treatment. Discussions were held about her fertility while on imatinib, her options for healthy childbearing, her individual prognostic factors, and her predicted survival with the treatment options that are currently available. She also had a comprehensive consultation with a fertility expert (the third author). She was offered several options, including contraception until after completion of her treatments, oocyte cryopreservation, and embryo cryopreservation. Based on the data (although limited) indicating that the diminished ovarian reserve while on imatinib is reversible, she decided to pursue pregnancy after her adjuvant treatment is completed.

FINANCIAL DISCLOSURE: The authors have no significant financial interest in or other relationship with the manufacturer of any product or provider of any service mentioned in this article.

$\Rightarrow \begin{aligned} & \text { For reference list, visit: } \\ & \text { cancernetwork.com/GIST_CaseStudy }\end{aligned}$

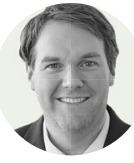

\section{Talmon}

is a professor in the Department of Pathology and Microbiology at the University of Nebraska Medical Center, specializing in gastrointestinal, renal, and placental pathology.

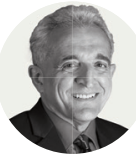

\section{Copur}

is a medical oncologist/ hematologist at Morrison Cancer Center, Mary Lanning Healthcare in Hastings, Nebraska, and is a professor at the University of Nebraska Medical Center. He is also an Editorial Advisory Board member at ONCOLOGY. of Nebraska Medical Center, where holds the James M.D. Distinguished Pathology Residency Program Director Chair.

\section{Wedel}

is a staff pathologist at Mary Lanning Healthcare.

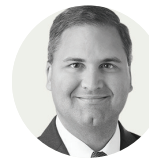

Nebraska.

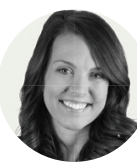

\section{Delaney}

is a double board certified obstetrician/gynecologist and reproductive endocrinologist.

She is currently in private practice at Omaha Reproductive Health Specialists, affiliated with Women's Methodist Hospital.

\section{Padussis}

is assistant professor, Department of Surgical Oncology, University of Nebraska Medical Center, Omaha,

a pathologist and Associate Professor in the Department of Pathology and Microbiology at the

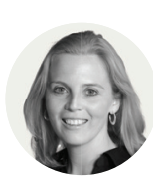

\section{Cushman-Vokoun}

is the medical director of the Molecular Diagnostics Laboratory at University of Nebraska Medical Center, and associate professor at the University of Nebraska Medical Center Department of Pathology and Microbiology, with research interest in the molecular pathology and testing of Gl-based malignancies. 


\section{Urology Times}

Leading research and analysis.

Practical advice.

\section{www.UrologyTimes.com}

From the publishers of 


\section{MEET OUR EXPERT}

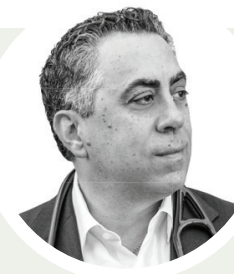

Bekait-Saab is

a medical oncologist at the Mayo Clinic in Phoenix, Arizona

\section{Ushering in the Era of Precision Medicine}

\section{"Next-generation sequencing should become a right, not a privilege"}

Treatment for patients with gastrointestinal (GI) cancer, specifically colorectal cancer (CRC), has undergone a major paradigm shift. Improvements in the understanding of cancer biology and the implementation of precision therapy have allowed oncologists to begin to deliver the proper treatment to the proper target, improving outcomes.

ONCOLOGY® recently sat down with Tanios S. BekaiiSaab, MD, of the Mayo Clinic in Phoenix, Arizona, to discuss the rapidly evolving treatment landscape of GI cancers, the most recent practice-changing studies, and the need to make genomic sequencing the standard of care for all patients with GI cancer.

\section{Can you discuss the Q. importance of next- generation sequencing in GI cancers?}

BEKAII-SAAB: I don't think anymore that genomic sequencing, next-generation sequencing (NGS), is a privilege; it should be a right. I think every single patient with a GI malignancy-specifically colon cancer, but [actually] all GI malignancies-has the right [to be sequenced], and we have to find every way possible to get them sequenced.

I need to understand what the rare mutational status of each patient is. What does BRAF look like? Do we have a $B R A F$ mutation? I want to know about HER2 amplification. I want to know about tumor mutational burden (TMB), [since] now we have an approval with high TMB for pembrolizumab (Keytruda). The cutoff point may be a little controversial; the 10 is not as meaningful but many of these patients with TMB of 15 and 20 are actually [having] great responses and responding really well to immune therapies. And in pancreas cancer, for example, I want to understand if there is a $B R C A$ mutation that may essentially be driving the cancer.

So, you can see the story forming here. But going back to colon cancer, I have at least 4 or 5 things I need to know from the get-go before I even start treating the patient. One could argue that, hey, let's get microsatellite instability-high (MSI-H) and RAS and $B R A F$ and HER 2 separately. But it turns out that if you get them separately, it's much more expensive than getting NGS. And with NGS, you actually get more than just the 3 or 4 things; you can get other things like NTRK fusions.

Now we know that NTRK fusions, which are incredibly rare, can be found. So, we're looking at needles in a haystack. The less you test, the less likely it is that you are going to find rare targets. So, we're not only looking for the common targets, but for the rare ones as well. And so, 1 snapshot with NGS should give you all the tools you need-or most of the tools, I should say, because there are still a lot of unknowns. But it should give you most of the tools you need to essentially plan ahead of time for that patient. What biologic am I going to start with? Is that a hopeful immunotherapy in this patient? Is chemotherapy the best choice, or is pembrolizumab the better choice? Should we enroll this patient on the BRAF V600E-mutated study, the follow-up to BEACON CRC (NCT02928224)?

Let me tell you why [identifying] HER 2 amplification is important. HER 2 amplification not only puts the patient on path for HER2-targeted strategies, which are being studied in multiple trials with multiple agents being developed, but it turns out that HER2 amplification tells us quite a bit about resistance to EGFR inhibitors. So, let's say you have a left-sided tumornaturally, you would think this patient should do great on an EGFR inhibitor. But it turns out that if the patient has a HER2 amplification, which occurs in about $4 \%$ of the patients, that patient will not respond to an EGFR inhibitor. And in fact, they progress very rapidly. So, all of these things-MSI-H, TMB, RAS, BRAF, HER2, plus a slew of others-can be a part of this 
NGS, this genomic profiling of the tumor. So, [again,] I think at this point in time, NGS should become a right and not a privilege, and all patients should have access to it. And the sooner the better, because it sets us on a much better track in terms of identifying the best options for patients with GI cancers.

\section{. What is driving the current progress in the field? Is it the increased testing?}

BEKAlI-SAAB: The answer is not just testing alone; testing was so random in the past that you couldn't make sense out of it. We also didn't have any agents or studies being looked at in the different spaces. Rather, I think what's energizing the field is the fact that while testing is being done, we're understanding more, and with more clarity, about what these different targets mean, what these different expressions mean, and how we can go after them. Six or 7 years ago it started with MSI-H cancer; we started seeing those results [from immune-based therapy] percolating in the later line[s of therapy] and moved [those therapies] to the first line, which just transformed the way we treat this disease. The biology that we're learning about starts in the later stage, then moves to the first line, and also ultimately moves to the adjuvant setting. So, we're starting to transform this cancer earlier and earlier. I think in 3 to 5 years, survival, for many patients, is going to be the equivalent of a cure, when we do things earlier on and the right way. A lot of work is still ahead of us, though, with tons of information [to work through]. This is where meetings like ISGIO [International Society of
Gastrointestinal Oncology] [are so important, to] keep you up to date with where the science is, what's changing practice today, what is likely to change practice tomorrow, and what we should be looking at after tomorrow.

\section{A. Can you tell me a little more about the ISGIO conference? What will we see at this year's meeting? This is your first year as the program director.}

BEKAII-SAAB: The meeting this year is certainly revamped. We're going to be changing a few things and keeping some things that worked in the past, especially the focus on fellow education and fellows' involvement. We're also diversifying the chairs of the different sessions quite a bit, ensuring [more] diversity, including representation from multiple institutions. We'll also introduce some debate-style crossfire and other elements that will keep it quite interactive. But we also want to preserve the spirit of ISGIO. That will essentially ensure that it's a meeting where not just fellows, but young faculty, early in their career, will not only be able to be involved but [will] be quite educated [from the discussions of the meeting], in terms of how they practice and in terms of understanding the evolving landscape of research and standard-of-care therapies in various GI malignancies.

\section{What are some of the key studies that have come out in the past year or two that have been practice-changing within the field?}

BEKAII-SAAB: I think that [the results] of KEYNOTE-177 (NCT02563002) are among the biggest stories over the last few years-at least in colon cancer. We've known for a while that in this subgroup of patients with MSI-H metastatic CRC, we have up to $30 \%$ to $40 \%$ of patients who have a response to pembrolizumab in the refractory setting. For most of these patients, the response is quite meaningful. What I mean by that is it's not just the level of response-which in many patients is actually a complete response-but the durability of the response as. About $20 \%$ to $30 \%$ of patients continue to respond, even after you stop the treatment at 2 years, and then continue to be in remission. So, the natural question leading to KEYNOTE-177 was, 'We see these incredible responses in the later lines-can we bring this up to the first line?' And I'll be frank with you, in my practice, I actually changed-even before [the results of] KEYNOTE-177 [were published]—to using pembrolizumab in the first line for patients with MSI-H tumors. And my thought was originally that, you know, some of these patients, $20 \%$ to $30 \%$ of them, will never need chemotherapy in their lifetime. They respond so well to these agents; I don't want to wait too long before [using it].

And so, I was very pleased with the results of the study, which essentially showed that indeed, in about one-third of the patients at least, if not more, pembrolizumab induces a significant response. [The results] essentially transform this disease from one where we have to try chemotherapy first and fail before we go to immunotherapy, to patients now getting immunotherapy first. And then, as I said, for 30\% to $40 \%$ of those patients-and I don't like to use the word "cure" loosely in stage IV disease-this is the equivalent of a cure. Those patients end up in remissions that are durable, and they may never ever see chemotherapy in their lifetimes. So that's great.

Now survival—we're still waiting on that. There are 2 possible scenarios. The first scenario is what a lot of people are predicting: Survival won't be much different between pembrolizumab given first versus chemotherapy being given first. The reason, [according to] a lot of folks, is, well, when you give chemotherapy first when patients progress, they will all have access to pembrolizumab and therefore most of them will be crossed over to immunotherapy. So, the thought is that, whether you get it first or later, it won't change the overall survival much. On the other hand, a small group of folks thinks that actually, we're probably going to see a survival benefit. It may not be as big as the PFS, but it will be there and it will be meaningful. Their reasoning is, since this is appears to be a game-changer, the sooner you change, or the sooner you attack the biology of the cancer, the better you will do on the long run and, therefore you will affect overall survival positively. I'm kind of closer to this camp, but I'll tell you that either way, I frankly don't care what the survival results are going to be. We know that they're not going to be detrimental and when I have about one-third to $40 \%$ of my patients go on pembrolizumab for a couple of years, and they'll be in durable complete remission and may never see chemotherapy in their lifetime, I think that's an achievement.

So, if the survival is positive, 
that's great. If the survival is borderline better, with pembrolizumab versus some sort of chemotherapy, when you look at the big story, prolonging their likelihood of not progressing, with a quality of life that's much better, I think it's a no-brainer. This has changed the landscape of how we treat MSI-H metastatic CRC. But let me even go further: I would say this has transformed the way we treat MSI-H tumors, period, beyond just metastatic CRC. [In other words], if I have a gastric cancer or a pancreas cancer or a cholangiocarcinoma patient who has MSI-H, I will treat them with pembrolizumab first, before even trying to treat them with chemotherapy. I do believe that the results, as we've seen in the later lines, are consistent regardless of anatomic site. It doesn't matter where the cancer starts; it's the MSI-H phenotype that's essentially determining the level of response. So that's [practice-changing]. I think that is an incredible study that just changed the way we think about those patients- $4 \%$ of all our patients with colon cancer. That's a lot of patients in a very common disease. It gives us a lot of hope that we're curing patients in stage IV disease with this modality.

The other trial that comes to mind is BEACON CRC (NCT02928224), a phase 3 , randomized trial with encorafenib plus cetuximab plus or minus binimetinib versus irinotecan and cetuximab in patients with refractory $B R A F$ V600E-mutated [tumors]. What we've seen in this study is that the biologic combo, [versus chemotherapy plus cetuximab], is improving survival and overall survival. Up to $25 \%$ of patients will have a meaningful response, and patients' quality of life is significantly better, with less toxicity. So that now is a standard for our patients with CRC and BRAF V600E mutations. We talked about this with KEYNOTE-177 and now with BEACON CRC; it is essentially leading the way to move these combination of agents into the first line and into the adjuvant setting, ultimately.

Another study that I think changed or transformed the way we treat patients, with caveats, is POLO (NCT02184195), which looked at patients with pancreatic cancer with germline BRCA1/2 mutations. The results showed that if you treat patients with a platinum-based therapy and then, after at least 16 cycles of exposure [if they're stable and have had a response, they take] olaparib [Lynparza], which is a PARP inhibitor, their progression-free survival is significantly improved. That study was transformative, in a sense; it doesn't necessarily change practice for most patients, but for the first time, in pancreatic cancer, we have a biologic option that was shown to essentially prolong progression-free survival and [patients had] a response to it. We never had that in pancreas cancer: to have a target and an agent that goes after the target. So that is transformative. That tells us that biology drives cancer, and when you identify the driver, you can actually flip a switch or offload the switch in a way to essentially establish some form of remission and for some, it's a durable remission.

\section{FINANCIAL DISCLOSURE: The}

authors have no significant financial interest in or other relationship with the manufacturer of any product or provider of any service mentioned in this article.

\section{KEY QUESTION}

You've said that the field of GI cancer

treatment is $\mathbf{2}$ to $\mathbf{3}$ decades behind

other forms of cancer in terms of

biologic and immune-based therapy. Do you feel like the field is starting to catch up?

BEKAII-SAAB: We remain a little bit behind, but we are catching up, and we're catching up quickly. And that's good news. For 20 years in colon cancer, we wasted a lot of energy debating whether fluorouracil plus oxaliplatin (FOX) or folinic acid plus fluorouracil plus irinotecan (FOLFIRI) is best, and whether bevacizumab or anti-EGFR inhibitors should be [used] first. [Fortunately,] that debate is done with, and we're moving essentially to an era where the question has become, What do I do with my MSI-H patient? Do I even need to consider chemotherapy? What do I do with a BRAF V600E-mutated patient? What do I do with my HER2-amplified patient? What do I do with the KRAS patient? What do I do with a lot of the other things that I, at this point in time, don't understand? How can I channel the patient toward a clinical trial that's specific for them? We're starting to break down common cancers and all these subgroups.

So, germline testing for every single one of these patients is needed. And we need to look at somatic alterations, as well, with NGS, because that changes how we treat our patients. If a patient has a BRCA mutation-whether it's germline or somatic-which is about $5 \%$ to $8 \%$ of patients, that patient needs to go on a platinum-based therapy. It's important to keep in mind that these findings have implications not only for targeted agents, but, say, in pancreas cancer, it also helps you understand whether you use a platinum first or a taxane.

So, we're catching up quickly and starting to make sense of all this biology that we've been trying to learn over the past 20 years. And today, thankfully, we are starting to understand how to place a lot of these agents into these baskets that are transforming how we treat our patients. And the great thing is, this is only the tip of the iceberg. We're learning so much about these hidden targets, and so many agents are either being looked at or will be looked at. Ultimately, these agents will transform how we treat colon cancer, and GI cancers overall. So, we're catching up, and I think in the next 3 to 5 years, we'll probably be ahead of the curve again. 


\section{Challenges of Treating a Patient With Advanced Prostate Cancer During the COVID-19 Pandemic}

Lucia Carril-Ajuria, MD'; Yuly A Remolina-Bonilla, MD²; Alberto Carretero-González, MD'; Maricruz Martín-Soberón, MD; Daniel Castellano, MD'; Christianne Bourlon, MD²; Guillermo de Velasco, MD, PhD'; Maria T Bourlon, MD²

'Department of Medical Oncology, Hospital Universitario 12 de Octubre, Madrid, Spain.; ${ }^{2}$ Department of Hematology and Oncology, Instituto Nacional de Ciencias Medicas y Nutrición Salvador Zubirán, Mexico City, Mexico.

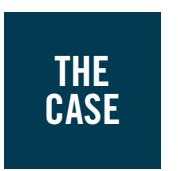

\section{A 78-year-old man had a} medical history of hypertension, atrial fibrillation, chronic kidney disease, and metastatic castration-resistant prostate cancer (CRPC). He had progressed to first-line therapy for CRPC with abiraterone plus androgen-deprivation therapy (ADT) and as second-line therapy he was being treated with docetaxel, with biochemical progression in his last prostate-specific antigen measurement.

He was admitted to the hospital on April 2020 , in the middle of the coronavirus disease 2019 (COVID-19) pandemic, because of painful bone lesions and deterioration of renal function. His fourth chemotherapy cycle and monthly zoledronic acid had been administered 4 weeks prior to admission.

Four days into this hospitalization, the patient developed respiratory symptoms characterized by shortness of breath and cough. Pulse oximeter confirmed by blood gas analysis revealed hypoxemia, requiring supplemental oxygen via nasal cannula at $2 \mathrm{~L} / \mathrm{min}$ to maintain an oxygen saturation level of more than $90 \%$. A chest $\mathrm{x}$-ray compared with the prior one taken a month ago showed bilateral patchy opacities predominantly on the right lung (Figures 1A and B). Nasopharyngeal swab specimens were positive for severe
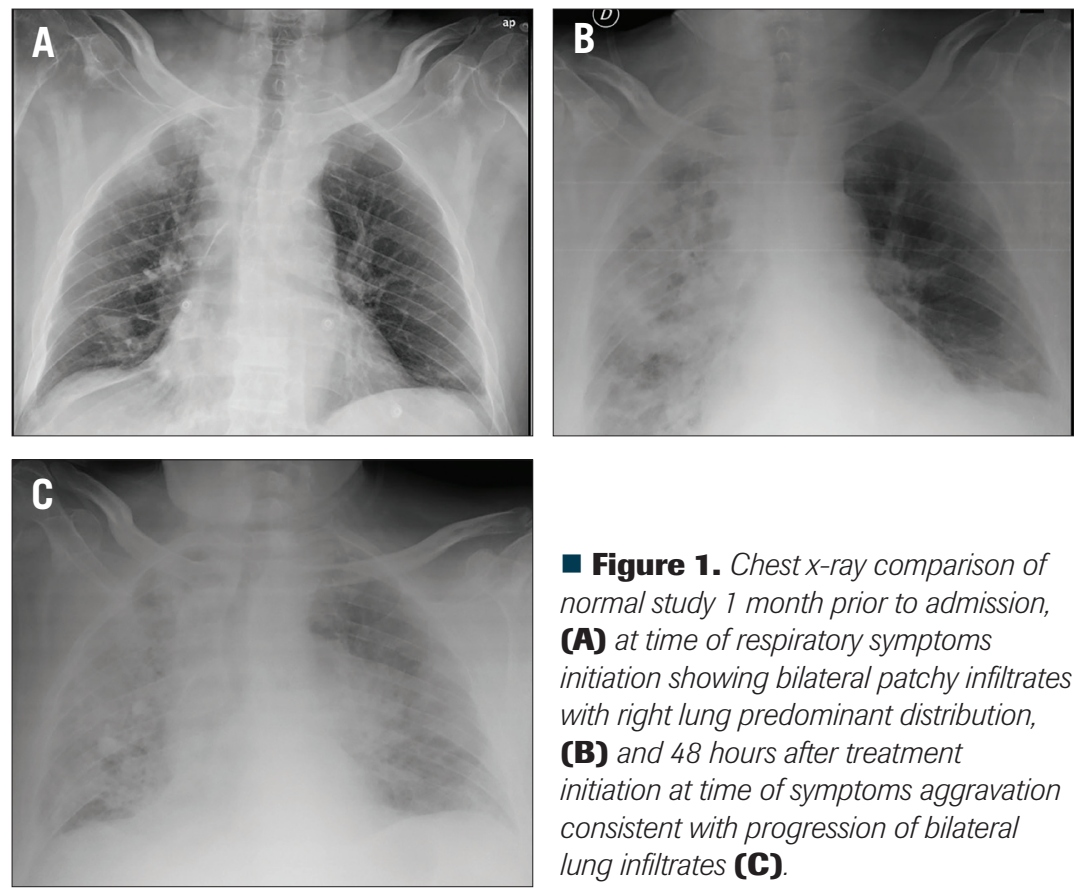

- Figure 1. Chest $x$-ray comparison of normal study 1 month prior to admission, (A) at time of respiratory symptoms initiation showing bilateral patchy infiltrates with right lung predominant distribution, (B) and 48 hours after treatment initiation at time of symptoms aggravation consistent with progression of bilateral lung infiltrates (C).

\section{Which of the following statements are true about cancer and COVID-19?}

\begin{abstract}
A. Patients with cancer have an increased risk of infection and related complications.
\end{abstract}

B. Transmembrane protease serine 2 (TMPRSS2) inhibition may be a protective factor for infection in patients with prostate cancer.
C. Predictive factors for mortality in patients with cancer are similar to those in all patients with COVID-19.

D. Palliative care delivery has changed with this pandemic.

E. All of the above are true. 


\section{CORRECT ANSWER: E. All of the Above}

Continued from page 317

acute respiratory syndrome coronavirus

2 (SARS-CoV-2) by real-time reverse

transcriptase-polymerase chain reaction

(rRT-PCR).

Additional laboratory tests revealed leukocytosis ( 14.900 cells/ $\mu$ l), lymphopenia (600 cells $/ \mu \mathrm{l}$ ), elevated C-reactive protein (31 $\mathrm{mg} / \mathrm{dL})$, elevated lactate dehydrogenase (580 U/L), and worsening of kidney function: creatinine 2.95 $\mathrm{mg} / \mathrm{dL}$ (baseline creatinine, $1.70 \mathrm{mg}$ / $\mathrm{dL}$ ), consistent with diagnosis of severe bilateral COVID-19 pneumonia with acute respiratory failure.

Treatment with antibiotics with aztreonam, ciprofloxacin, and linezolid (because of his $\beta$-lactam allergy); antiretroviral therapy with lopinavir-ritonavir; and low-molecular-weight heparin (LMWH) thromboprophylaxis was started. After 48 hours of full medical therapy, his clinical condition deteriorated and his oxygen requirements increased. A follow-up chest $x$-ray revealed worsening bilateral patchy infiltrates (Figure 1C). A multidisciplinary discussion among the internal medicine, pulmonology, intensive care, and medical oncology teams was held. The patient was not considered a candidate for mechanical ventilation due to a shortage of intensive care unit beds as a result of the pandemic, neoplastic disease in progression with second-line therapy, poor performance status, and coexisting comorbidities. He was sent home with palliative care with quarantine precautions for himself and his family, and died 3 days later due to respiratory failure.

\section{Discussion}

COVID-19 is a viral respiratory illness caused by SARS-CoV-2 and was declared a global pandemic on March 11, 2020.
It has affected all medical disciplines. Treating patients effectively within this health crisis has now become a major challenge for clinicians. Cancer care delivery is no exception, as all aspects of its practice-from screening to palliative care-have had to be modified, with inevitable consequences for medium- and long-term prognosis. ${ }^{1,2}$ Worldwide, the practice of oncology has now changed. ${ }^{3}$ Oncologists face challenges such as delays in surgical procedures, preferential use of oral agents instead of parenteral therapy regimens, and ensuring that telemedicine visits are available. Meanwhile, patients are experiencing travel restrictions on account of quarantine policies, and are fearful of visiting hospitals because of the exposure risk and unavailability of outpatient services, among other concerns. ${ }^{4}$

Present data suggest that cancer patients possess an increased risk for severe COVID-19 and its complications, including death. ${ }^{5-8}$ Initial information from China revealed a higher infection rate among cancer patients compared with all COVID-19 cases $(0.79 \%$ vs $0.37 \%$, with an odds ratio (OR) of 2.31 (95\% CI, 1.89-3.02) ${ }^{8}$, and other studies describe higher infection rates approximately 6 times greater in patients with malignancy compared with the general population. ${ }^{9,10}$

In Chinese studies, $13.4 \%$ to $41.3 \%$ of COVID-19 cases in patients with cancer were believed to occur through hospital-associated transmission. The most common neoplasm associated with this infection was lung cancer $(22.4 \%$ $28 \%$ of cases). One- to two-thirds of patients (39.2\%-64.2\%) had coexisting chronic diseases. Almost all patients (> $94 \%$ ) had abnormal findings on computed tomography (CT) at COVID-19 disease presentation, and the proportion of patients who had received oncologic treatment within the last month was $21.4 \%$ to $34.3 \%$. Acute respiratory distress syndrome developed in $15.6 \%$ to $28.6 \%$ of patients with cancer, with mechanical ventilation requirement and intensive care unit (ICU) admission rates of $35.7 \%$ to $41.8 \%$ and $21.4 \%$, respectively. Compared with the overall population, patients with cancer developed more severe illness $(47.8 \%$ $53.6 \%$ vs $19 \%$ ), had a more rapid deterioration (13 vs 43 days), and had a tenfold higher case-fatality rate $(25 \%$ $28.6 \%$ vs $2.3 \%) . .^{5-8,11}$ Patients receiving any modality of oncologic treatment within 14 days of COVID-19 diagnosis demonstrated an increased risk for admission to an ICU, mechanical ventilation requirement, or death (HR, 4.1; 95\% CI, 1.1-15.3; $P=.037$ ), whereas patients with a patchy consolidation on CT at presentation showed an increased risk of complications or death (HR, 5.4; 95\% CI, 1.5-19.7; $P=.010) .^{6}$

Currently, the United States has the largest number of COVID-19 cases of all countries, with New York City being the country's first epicenter. The city's data incorporated new predictive factors for adverse outcomes in patients with cancer who also have COVID-19. The highest case-fatality rate occurred in those with lung cancer $(55 \%)$. Genitourinary and breast cancers were associated with lower mortality $(14 \%$ and $15 \%$, respectively). Factors related with increased mortality included the following: age (76 vs 66 years, $P=.0006$ ); comorbidities, such as hypertension $(P=.047)$; coronary artery disease $(P=.012)$; chronic heart failure $(P=$ $.019)$; chronic lung disease $(\mathrm{P}<.001)$; and metastatic disease $(P=.06)$. Laboratory findings such as anemia $(P=.047)$, higher absolute neutrophil counts $(\mathrm{P}=$ .017), elevated D-dimer levels $(P=.002)$, lactic acid $(P=.001)$, and lactate dehydrogenase (LDH; $\mathrm{P}=.01$ ) were significantly associated with worse survival. In the multivariate analysis of factors asso- 
ciated with case fatality, being aged 65 or younger was a protective factor for death (OR, 0.23; 95\% CI, 0.07-0.61; P $=.005)$, and factors such as ICU admission (OR, 4.83), D-dimer (OR, 1.15), lactic acid (OR, 1.99), LDH (OR, 1003), and comorbidity score (OR, 1.52) were all related to a poor prognosis with statistical significance. New York City data also reaffirm that cancer patients have a 2.45 -fold elevated fatality rate $(\mathrm{P}<.001)$ compared with the general population. ${ }^{12}$

Therefore, statements $\mathrm{A}$ and $\mathrm{C}$ are correct options.

Regarding prostate cancer, current information is based on Italy's experience, which revealed that all male patients with cancer had an increased risk of SARS-CoV-2 infection (OR, 1.79; 95\% CI, 1.62-1.98; $P<.0001)$ with higher hospitalizations and mortality rates. When the prostate cancer population ( $\mathrm{n}=118$ ) was divided according to treatment with ADT versus no ADT, a significantly higher risk of COVID-19 was identified in the non-ADT-treated group (OR, 4.05; 95\% CI, 1.55-10.59; P $=.006)$. Additionally, when other types of cancer were compared with the ADT group, an increased risk was sustained (OR, 5.17; 95\% CI, 2.02-13.40; $P=$ $.001) .{ }^{13}$ This may be explained through the expression of TMPRSS2-mainly in the prostate, but also in the lung-which is upregulated by androgen receptor, facilitates viral entry and replication, and cleaves angiotensin converting enzyme 2 for augmented viral entry. ${ }^{14,15}$ Androgen sensitivity could be a determinant factor in COVID-19 severity and ADT downregulates this phenomenon. ${ }^{16}$ Thus, option B is also a valid statement.

The data in relation to COVID-19 and cancer must be interpreted with caution because of the retrospective nature and heterogeneity of the studies. In addition, these reports do not include information regarding do-not-resuscitate or do-not-intubate orders, which could impact mortality and severe events calculation.
COVID-19 has exposed the weaknesses of health care systems in several areas including palliative care, which has faced many challenges during this pandemic. ${ }^{17}$ One example is Italy, where the requests of home care assistance doubled in a system that was already saturated. There also was a lack of the equipment required to protect patients and health care workers, limited patient follow-up at home, and, in some cases, a cessation of activities by hospices. ${ }^{18}$ In Singapore, palliative care teams were disassembled so that physicians could work on the front lines. ${ }^{19}$ Furthermore, prohibiting the presence of a family member resulted in many patients dying alone, which has profoundly affected the palliative care carried out by specialists, in which a multidisciplinary approach and family involvement is essential.

For patients with cancer, the management of COVID-19 needs to be adjusted according to the type of cancer, disease stage, expected survival, line of treatment, and previous performance status. ${ }^{20}$ These have always been important when patients with cancer face an acute illness; however, they become more crucial when the patient and the system are unable to adapt, and resources are limited, as has been the case during this pandemic. Currently, clinicians must discuss care goals in advance with their patients who have cancer and allow them to make their own decisions. ${ }^{17,21}$

Therefore, option D is also correct.

Would the treatment the 78-year-old patient received have been different had his last progression not occurred during the pandemic? As clinicians, we cannot avoid thinking about this question. Perhaps the outcome would have been different. For instance, the access to an ICU would not have been so restricted, his condition could have improved, and he could have reached a better performance status that might have allowed for further therapeutic interventions. Nowadays, there are many treatment options for metastatic CRPC; hence, the patient would have had an alternative for a third-line treatment such as enzalutamide or cabazitaxel.

In conclusion, our clinical case is a good example of the combination of several predictive risk factors such as age, coexisting comorbid conditions, laboratory findings, and recent oncological treatment (chemotherapy) that lead to worse outcomes in patients with cancer who have COVID-19.

FINANCIAL DISCLOSURE: The authors have no significant financial interest in or other relationship with the manufacturer of any product or provider of any service mentioned in this article.

\section{CORRESPONDING AUTHOR:}

Maria T Bourlon MD, MHS

Vasco de Quiroga No. 15., Belisario Dominguez Sección XVI, Tlalpan, C.P. 14080, Ciudad de

México, México

maitebourlon@gmail.com

\section{ABOUT THE SERIES EDITORS:}

Maria T. Bourlon, MD is Associate Professor, Head Urologic Oncology Clinic, National Researcher. Instituto Nacional de Ciencias Médicas y Nutrición Salvador Zubirán. Mexico City, Mexico. She is also a Member of ASCO's IDEA Working Group.

E. David Crawford, MD, is Chairman, Prostate Conditions Education Council; Editor in Chief, Grand Rounds in Urology; and Professor of Urology, University of California San Diego, La Jolla, California.

\section{KEY REFERENCES}

1. Dinmohamed AG, Visser O, Verhoeven $\mathrm{RHA}$, et al. Fewer cancer diagnoses during the COVID-19 epidemic in the Netherlands. Lancet Oncol. 2020;21(6):750-751. doi:10.1016/S14702045(20)30265-5

2. The Lancet Oncology. Safeguarding cancer care in a post-COVID-19 world. Lancet Oncol. 2020;21(5):603. doi:10.1016/S14702045(20)30243-6

3. Waisberg F, Enrico D, Angel M, Chacón M. Cancer treatment adaptations in the COVID-19 era. JCO Oncol Pract. 2020 Jun;16(6):305-307. doi:10.1200/op.20.00218

4. Gao Z, Yang Y, Ding C, et al. Oncologist's perspective: when cancer encounters COVID-19. Oncologist. Published online May 15, 2020. doi:10.1634/theoncologist.2020-0296

5. Liang W, Guan W, Chen R, et al. Cancer patients in SARS-CoV-2 infection: a nationwide analysis in China. Lancet Oncol. 2020;21(3):335-337. doi:10.1016/S1470-2045(20)30096-6 
6. Zhang L, Zhu F, Xie L, et al. Clinical characteristics of COVID-19-infected cancer patients: a retrospective case study in three hospitals within Wuhan, China. Ann Oncol. 2020;31(7):894-901. doi:10.1016/j. annonc.2020.03.296

7. Zhang HY, Wang LW, Chen YY, et al. A multicentre study of 2019 novel coronavirus disease outcomes of cancer patients in Wuhan, China. medRxiv. Published online April 15, 2020. doi:10.1101/2020.03.21.20037127

For full reference list, visit cancernetwork.com/COVID19_prostate

\section{Carril-Ajuria}

Department of Medical Oncology, Hospital Universitario 12 de Octubre, Madrid, Spain.

\section{Remolina-Bonilla}

Department of Hematology and Oncology, Instituto Nacional de Ciencias Medicas y Nutrición Salvador Zubirán, Mexico City, Mexico.

\section{Bourlon}

Department of Hematology and Oncology, Instituto Nacional de Ciencias Medicas y Nutrición Salvador Zubirán, Mexico City, Mexico.

\section{Bourlon}

Department of Hematology and Oncology, Instituto Nacional de Ciencias Medicas y Nutrición Salvador Zubirán, Mexico City, Mexico.

\section{Velasco}

Department of Medical Oncology, Hospital Universitario 12 de Octubre, Madrid, Spain.

\section{Carretero-González}

Department of Medical Oncology, Hospital Universitario 12 de Octubre, Madrid, Spain.

\section{Martín-Soberón}

Department of Medical Oncology, Hospital Universitario 12 de Octubre, Madrid, Spain.

\section{Castellano}

Department of Medical Oncology, Hospital Universitario 12 de Octubre, Madrid, Spain.

\section{CLINICAL TRIALS IN PROGRESS}

\author{
PANTHER TRIAL
}

TITLE : Prospective Study of Apalutamide and Abiraterone Acetate iN ChemoTHerapy-Naïve MEn With mCRPC Stratified by Race (PANTHER; NCT03098836)

BACKGROUND: African American or Black men are underrepresented in nearly all therapeutic trials in advanced prostate cancer. Our recently concluded AbiRace trial (Clinicaltrials.gov NCT01940276) enrolled 50 Black and 50 white men, revealing provocative differences in prostatespecific antigen (PSA) response, progression, and adverse effect rates.

The PANTHER trial follows a similar format with men with metastatic castration-resistant prostate cancer (mCRPC) stratified by race. All patients receive free treatment with abiraterone acetate, an androgen ligand synthesis inhibitor, and with apalutamide, an androgen receptor inhibitor, to enhance androgen-deprivation therapy (ADT). Our goal is to determine if there are any race-driven differences in the efficacy of this combination.

The end points are radiographic progressionfree survival and PSA kinetics, time to nadir, and percentage of men who achieve a PSA less than 0.1 $\mathrm{ng} / \mathrm{mL}$. We will also estimate the rate of objective response and the incidence of bone flares; measure safety and tolerability, particularly incidence and grade of hypertension; and evaluate overall survival in Black and White men. Exploratory end points revolve around development and validation of genetic biomarkers for disease prognosis and determination of treatment efficacy.

PANTHER is an investigator-initiated trial, funded by Janssen (investigational new drug application \#134175; NCT03098836). This study has opened within the Department of Defense Prostate Cancer Clinical Trials Consortium.

INCLUSION CRITERIA: Self-reported Black or White men are eligible if they possess ICRPC greater than $2.0 \mathrm{ng} / \mathrm{ml}$. Evidence of mCRPC in the setting of ongoing ADT should be met by at least 1 of these criteria: (1) absolute rise in PSA of 2.0 $\mathrm{ng} / \mathrm{mL}$ or an increase or more than $25 \%$ from the nadir; (2) minimum of 2 consecutive rising PSA levels with an interval of 1 or more weeks between each PSA level; (3) CT- or MRI-based evidence of disease progression (soft tissue, nodal, or visceral scan lesion compared with the most immediate prior radiologic studies. Patient should have adequate laboratory parameters. Prior systemic chemotherapy, immunotherapy, or treatment with abiraterone acetate, enzalutamide, apalutamide, galaterone (TOK-001), orteronel (TAK-700), or similar agent is not allowed. Patients with prior chemotherapy for hormone-sensitive prostate cancer are allowed.

\section{PATIENT ACCRUAL INFORMATION}

- Accrual goal: 100

- Percent accrued: $88 \%$ accrual completed. Forty-nine White and 39 Black men have been enrolled so far, with 11 slots remaining.

STUDY SITES: A total of 8 sites are open to accrual: Duke University Medical Center, Duke Cancer Network, Virginia Oncology Associates, Karmanos Cancer Institute, Spartanburg Veterans Administration Medical Center, University of North Carolina Lineberger Cancer Center, Tulane University, and Chesapeake Urology Research Associates. disease based on RECIST 1.1 criteria with PSA disease progression); or (4) at least 1 new bone

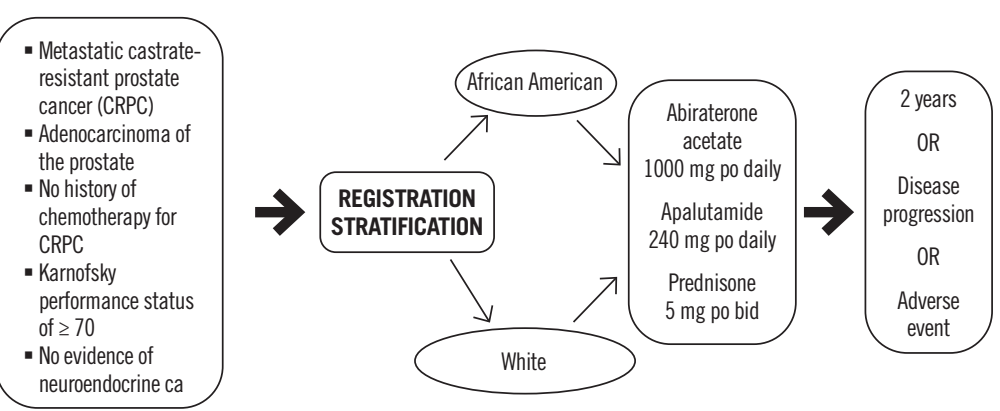

\section{Co-principal investigators:}

Daniel George, MD

905 LaSalle Street, GSRB1 Room 3017A, Durham, NC 27710. Tel: 919-668-4615, Fax: 919-668-7117, daniel.george@duke.edu 


\title{
Predictive Biomarkers for Immunotherapy Response Beyond PD-1/PD-L1
}

Sourat Darabi, PhD, MS'*; David R. Braxton, MD'; Burton L. Eisenberg, MD'1,2; and Michael J. Demeure, MD, MBA',3 ${ }^{7}$ Hoag Family Cancer Institute, Newport Beach, CA; ${ }^{2}$ University of Southern California, Los Angeles, CA; ${ }^{3}$ Translational Genomics Research Institution, Phoenix, AZ

\begin{abstract}
Advances in immuno-oncology over the last several years have led to FDA approvals of novel agents. As our understanding of immune response and its checkpoints has evolved, further advances have been made in treatment for several cancer types. To predict a response to immunotherapy, the initial biomarkers used were expression of the PD-1 receptor and PD-L1, as assessed by immunohistochemistry. More recently, predictive biomarkers have included microsatellite instability, DNA mismatch repair, and tumor mutational burden. Although these markers may be clinically relevant in predicting an immunotherapy response, cancer immunotherapy fails some patients. Improved understanding of the human immune system is necessary, as is a careful evaluation of the methods used to predict and assess response to immunooncology treatments. With the application of therapeutic immune-modulating agents, more comprehensive assays, and associated bioinformatics tools to accurately assess the tumor microenvironment, we may better predict responses to immuno-oncology agents and the ever-increasing complexity of their clinical use.
\end{abstract}

\section{Background}

Progress in cancer immunotherapy is advancing rapidly, primarily as the result of better understanding the complexities of immune system modulations, both in sickness and health. Discoveries in cancer immunotherapy and immuno-oncology's successful application in various tumor types led to the award of the 2018 Nobel Prize in Physiology or Medicine to James P. Allison, PhD, and Tasuku Honjo, MD, PhD. ${ }^{1}$ The US FDA has approved several immuno-oncology agents since 2011. Despite the notable successes, however, many patients don't benefit from cancer immunotherapy, in part due to the integrated nature of the human immune system and its interaction with cancer cells. ${ }^{2}$
The hallmarks of cancer include genetic and epigenetic changes that lead to the transformation of normal cells, the interaction of these cancer cells with the immune environment, and the cancer cells' capability to evade the immune response. ${ }^{3}$ To clarify how immune resistance works, it is helpful to delineate the causative contributors to immune sensitivity and cancer. Antitumor activity of the immune system is considered to be cancer immunosurveillance, and this surveillance may be interrupted by evading the immune response or suppressing its effect. ${ }^{3,4}$ Careful assessment of the methods to predict and assess the response to immunotherapy beyond currently used assays is necessary. Improved understanding of the mecha- nisms involved in inducing and escaping tumor immune response-and especially the role of the tumor microenvironmentwill inform the development of therapeutic agents.

\section{The Cancer Immunity Cycle and Tumor Immune Microenvironment Phenotypes}

The cancer immunity cycle is the process by which cancer cells release antigens that are processed and presented by antigen-presenting cells, with subsequent T-cell priming and activation. The activated T-cells then migrate out of the lymph nodes into systemic circulation; they traffic to the tumor site, infiltrate the tumor, and (perhaps) cause cytotoxic T-cell tumor killing. ${ }^{5}$ Each 
step in this process can be influenced by tumor intrinsic factors, host factors, host microbiome, environmental factors, and prior treatments. ${ }^{6}$ For instance, if a tumor does not produce antigens, then an immune response will fail to be initiated. ${ }^{7}$ Likewise, if a host cannot mount an immune response due to immunosenescence (age-related deterioration of the immune system), the tumor will evade eradication even if an abundant antigen is present. ${ }^{8}$

There are 3 tumor immune microenvironment phenotypes. First, inflamed (hot) tumors show a high degree of cytotoxic T-cell infiltration and elaborate proinflammatory cytokines. Higher response rates to anti-PD-1/PD-L1 therapy in inflamed tumors have been reported. ${ }^{6}$ While an inflamed microenvironment is thought to be necessary for effective checkpoint blockade, it is apparent that its presence alone does not guarantee clinical responses. Second, immune-excluded (warm) tumors show T-cells within the tumor stroma but little infiltration into the tumor. These peritumoral lymphocytes have been shown to be phenotypically distinct from tumor-infiltrating lymphocytes, and this microenvironment portends a diminished immunological and clinical response to anti-PD-L1/PD-1 therapy. 6, 9-11 Third, immune desert (cold) tumors show few or no T-cells within the microenvironment; they are unlikely to respond to anti-PD-L1/PD-1 therapy. ${ }^{6} \mathrm{Un}$ derstanding the components of the cancer immunity cycle and the tumor immune microenvironment phenotypes provide a framework for developing novel predictive biomarkers and treatment strategies within the immunotherapy development arena.

Among the most intriguing facets of the cancer immunity cycle currently being studied is the host systemic immune response. Every person has a homeostatic baseline of immune cell abundance and T-cell receptor diversity circulating within their peripheral blood, and this baseline differs significantly among individuals. ${ }^{12}$ This variation is overwhelmingly dictated by environmental factors, with only sparse contributions from heritable factors. ${ }^{12,13}$
Furthermore, as an individual ages, there are significant declines in naïve CD8+ T-cells and compensatory increases in central memory CD4+ T-cells. ${ }^{14}$ Because naïve CD8+ T-cells are the cell types necessary for recognizing new antigens, their decline is consistent with the observation of immunosenescence in older individuals. ${ }^{8}$ Immunosenescence also involves decreased T-cell receptor diversity ${ }^{15,16}$ and a broad decline in overall immune system activity. Seropositivity for cytomegalovirus (CMV) appears to significantly adversely impact the immune repertoire and may mediate immunosenescence; however, it is still unclear if CMV seropositivity impacts the effectiveness of immunotherapeutics. ${ }^{8,17}$

Significant evidence indicates that the diversity of the T-cell receptor repertoire is vital in the context of immune checkpoint blockade, or inhibition (ICI). The diversity and size of T-cell receptor clones responding to ICI have been shown to correlate with clinical responses and survival in patients with pancreatic cancer ${ }^{18}$ and metastatic melanoma. ${ }^{19}$ Similarly, peripheral lymphopenia has been shown to be present in $20 \%$ of patients with solid tumors, and it is associated with poor survival in multiple tumor types in the setting of ICI. ${ }^{20-22}$ Additionally, the amount of tumor present has been shown to be an important determinant of ICI failure, ${ }^{23}$ which suggests that in some patients, the immune system may be unable to expand sufficiently to accomplish tumor cell killing when faced with high tumor burden. This evidence supports the assertion that a significant proportion of patients inherently lack robust immune regulation and therefore will not benefit from current ICI strategies. Recent technological advances enabling measurement of the peripheral immune system and of the tumor immune microenvironment can inform strategies to replace an inadequate adaptive immune system with treatments such as cellular therapies (eg, chimeric antigen receptor T-cell therapy).

Even if a patient can mount a systemic immune response, the tumor may evade the immune system by a variety of tumor-intrin- sic mechanisms. Alterations in oncogenic signaling pathways, including TP53, PTEN, MYC, beta catenin, and LKB1, have been shown to mediate immune evasion in a variety of cancers via manipulation of factors important in T-cell recruitment and infiltration. ${ }^{24}$ Similarly, tumors may elaborate cytokines unsuitable to support a cytotoxic T-cell-mediated immune response. In a post hoc analysis of the IMVIGOR trial (NCT02108652) of atezolizumab in metastatic urothelial carcinoma, high levels of systemic and tumor microenvironment interleukin-8, a cytokine that typically serves to attract neutrophils, were found to correlate with reduced benefit of the PD-L1 inhibitor. ${ }^{25}$ Failures of the immune system at the systemic and microenvironment levels likely lead to cold or warm tumor immune microenvironment phenotypes.

\section{Known Biomarkers}

Tumor immunotherapy increases the antitumor response by the patient's immune system. This antitumor response involves different types of immune cells. In the past few years, ICIs have evolved and are now included in therapies for cancer types including non-small cell lung cancer (NSCLC), melanoma, bladder cancer, renal cell carcinoma, breast cancer, and endometrial cancer. ${ }^{26,27}$ ICIs target the key mediators of pathways that are involved in immune response. FDA-approved ICIs utilized for different tumor types include pembrolizumab, atezolizumab, avelumab, durvalumab, ipilimumab, and nivolumab. Several commercially available tests can identify biomarkers that indicate the level of response to immunotherapy. Detection of these predictive biomarkers assists in identifying patients for immunotherapy as well as those who may be resistant to such treatments. ${ }^{28}$

Cytotoxic $\mathrm{T}$ lymphocytes identify the tumor-associated antigens on cancer cells and destroy them. PD-1 is a membrane receptor protein on T-cells that induces cell death. PD-1 reduces antigen receptor activation by PD-L1 and PD-L2. PD-L1 presents on dendritic cells in activated 
TABLE. Selected Biomarkers of Immunotherapy Discussed, by Sample Type, Methodology, and Indication 6,18-19, 30, 66-67, 85-89

\begin{tabular}{|c|c|c|c|}
\hline Biomarkers of immunotherapy & Sample type & Methodology & Indication \\
\hline PD-1/PD-L1- IHC & TT & IHC/ Flow Cytometry & SOC \\
\hline Tumor mutational burden & TT & NGS & SOC \\
\hline DNA mismatch repair & TT & IHC/NGS & SOC \\
\hline Microsatellite instability & TT & NGS & SOC \\
\hline Somatic mutations & TT & NGS & SOC \\
\hline Circulating tumor DNA & PB & NGS & SOC \\
\hline Cytokines & PB & ELISA & Investigational \\
\hline Cellular peripheral immune response & PB & $\begin{array}{l}\text { Mass cytometry-time of flight } \\
\text { (CyTOF) }\end{array}$ & Investigational \\
\hline Circulating monocytes & PB & $\begin{array}{l}\text { High-dimensional single-cell mass } \\
\text { cytometry }\end{array}$ & Investigational \\
\hline Gut microbiome & Stool & Metagenomics & Investigational \\
\hline MicroRNA & Serum & NGS & Investigational \\
\hline Neutrophil/lymphocyte ratio & Blood or infiltrate tissue & Complete blood count & Investigational \\
\hline $\mathbf{T}$ cell receptor repertoire & $\mathrm{TT} / \mathrm{PB}$ & NGS & Investigational \\
\hline
\end{tabular}

ELISA, enzyme-linked immunosorbent assay; IHC, immunohistochemistry; NGS, next-generation sequencing; PB, peripheral blood; SOC, standard of care; TT, tumor tissue.

macrophages. PD-1 plays an important role in the mechanism of immune escape by binding to PD-L1 or PD-L2 and activating the signaling pathway in cancer cells that express PD-L1. ${ }^{4}$

Various methods are used to detect the expression of PD-L1. Immunohistochemistry (IHC) assays may be useful in determining the response to immunotherapy. Antibody clones to identify and quantify PD-L1 protein are 22C3, SP142, 28-8, and SP263 that are registered at FDA on Dako and Ventata platforms. ${ }^{29}$ Different clinical trials that led to the approval of the immuno-oncology agents used different assays to assess PD-L1 expression, so it is unclear if these assays are similar. In addition, intratumor heterogeneity, technical issues, time of biopsy, and variation in interpretation of "positivity" are current challenges in PD-L1 assays. ${ }^{29}$

Besides PD-1/PD-L1 expression analysis, the presence of microsatellite instabili- ty (MSI) is an important response marker. It is characterized by deficient DNA mismatch repair (MMR). The DNA MMR pathway includes MLH1, MSH2, PMS2, and MSH6 proteins, and when the pathway is defective (dMMR), it can be a measure to select patients for immunotherapy (Table).

\section{Tumor Mutational Burden}

A biomarker frequently used to predict whether a tumor might respond favorably to treatment with anti-PD-1 or anti-PDL1 immunotherapy is tumor mutational burden (TMB), defined as "a quantitative measure of the total number of somatic nonsynonymous mutations within the coding region of a tumor genome." 30 The hypothesis is that tumors exhibiting high TMB will expose more neoantigens that are capable of eliciting an immune response. Initially, TMB was determined using whole exome sequencing (WES) derived by next-generation sequence analysis of tumor samples and matching nontumor tissue. It is important to understand the evidence to support the use of $\mathrm{TMB}$, the techniques by which TMB is determined, and the limitations associated with this biomarker.

WES is uncommon in real-world practice. Rather, TMB is determined by interpreting data from large tumor sequencing panels that include only somatic genes. Determining TMB from such a panel is a normalized extrapolation expressed as the number of mutations per megabase (mut/Mb); however, there is variability among labs in how this is performed and interpreted. ${ }^{31}$ Differences arise in sample preparation and in sequencing techniques such as panel design, coverage and depth, bioinformatics pipelines, thresholds for variant calling, and cutoffs for calling high $\mathrm{TMB}$; these have all differed in studies reporting $\mathrm{TMB}$ as a biomarker of response 
to ICI. Matched tumor-normal sequencing allowed for accurate somatic variant calls among these studies, although fewer labs sequence normal matching DNA. Most, but not all, commercial labs used in clinical practice sequence large panels, each consisting of 300 to 600 genes. Panels often cover the entire coding regions, or a relevant regions of genes, or hotspots of known mutations. Large-panel sequencing does have advantages, including lower cost, decreased DNA requirements, and more rapid results when compared with WES. Therefore, when one must sequence a patient's paraffin-embedded (often small) tumor samples to provide timely results to guide therapeutic decisions, gene panels may be the best option.

Recent analysis suggests that targeted somatic gene panels, if they are sufficiently large (sequencing at least 315 genes, or 1.1 $\mathrm{Mb}$ ), do correlate well with TMB as determined by WES. ${ }^{32}$ However, the correlation may not hold equally well across all tumor types. Examination of FoundationOne CDx data compared with The Cancer Genome Atlas (TCGA) data for 33 tumor types determined that TMB estimation was reliable $\left(R^{2} \geq 0.75\right)$ in just 24 of 33 TCGA cancer types. ${ }^{33}$ After removing the cases of ultra-high TMB or hypermutated tumors, the correlation was worse. The reliability of TMB calls based on FoundationOne CDx was deemed adequate in melanoma, lung adenocarcinoma, cervical squamous cell cancer, head and neck squamous cell cancer, and stomach adenocarcinoma. In several other tumor types, though, the panel test was found to inadequately estimate TMB. Overall, across all tumor types studied, a meta-analysis of published critical trials examining the use of immuno-oncology agents showed a $47 \%$ risk reduction for death in patients whose tumors had high TMB when compared with those with low TMB.34 It is not clear, however, that all individual cancer types adhere to this observation. Cutaneous melanoma and NSCLC are most studied, and this may be because these tumors, compared with most others, have higher overall TMB. ${ }^{35}$

\section{Melanoma}

Malignant melanoma is among the tumor types exhibiting the highest TMB, likely due to the role of DNA damage caused by ultraviolet light in the pathogenesis of these tumors. ${ }^{36}$ Several studies have shown that patients whose melanoma tumors exhibit high TMB have prolonged survival when treated with immunotherapy drugs. ${ }^{37}$ Snyder et al (2014) analyzed the exomes of 64 patients with malignant melanoma and demonstrated that patients treated with ipilimumab whose tumors harbored more than 100 nonsynonymous mutations per exome had improved survival compared with those with fewer mutations $(P=.04) .{ }^{38}$ They used a bioinformatics pipeline that incorporated patients' human leukocyte antigen (HLA) type, major histocompatibility complex (MHC) class I binding prediction, and modeling of T-cell receptor binding to develop a neoepitope signature that was highly correlated with survival $(P=.002)$. Retrospective analysis of archival samples from an initial and a validation cohort representing a total of 65 patients using the FoundationOne CDx 315-gene panel showed that TMB was significantly higher in responders to anti-PD-1/PD-L1 therapy than in nonresponders $(P<.005) .{ }^{39}$ In the validation cohort, the responders' tumors had a median of 37.1 mut/Mb compared with 12.8 in nonresponders $(P=.002)$. Both progression-free survival (PFS) and overall survival (OS) were better for the group of treated patients whose tumors had a high TMB. A recent review by Krieger et al cited 40 studies that present outcome data for melanoma. ${ }^{40}$ Of these, only 3 studies reported TMB as a biomarker, including the study by Johnson cited previously. One of the retrospective studies (Yaghmour et al, 2016) included a small number of patients and used different commercial sequencing panels, but no difference in OS for patients with melanoma was observed, perhaps due to relatively small numbers $(P=.17) .{ }^{41}$ The other study (Roszik et al, 2016) predicted TMB from the 312 patients from the TCGA melanoma dataset. ${ }^{42}$ Results of another study (Forschner et al, 2019) showed that TMB in circulating tumor DNA (ctDNA) was higher in the patients who responded to combined PD-1 and CTLA-4 blockade. ${ }^{43}$ These investigators used a 710-gene panel in which all exonic and flanking intronic regions were sequenced. A high TMB was defined as more than $23.1 \mathrm{mut} / \mathrm{Mb}$. Interestingly, the authors found that a decrease in ctDNA to less than $50 \%$ was favorably associated with improved survival. A high predicted TMB correlated with survival in patients treated with ipilimumab or adoptive T-cell therapy. In melanoma, a low TMB does not preclude response to PD-1/PD-L1 response, and in and of itself, TMB has a poor predictive value in distinguishing patients whose tumors will or will not respond to ICI therapy. ${ }^{44}$

\section{Lung Cancer}

In 2015, the first study to show benefit from PD-1 blockade in NSCLC was KEYNOTE-001 (NCT01295827), in which 495 patients were treated with pembrolizumab. The benefit was correlated with PD-L1 expression of greater than $50 \%$ in the tumors; however, TMB was not assessed or reported. ${ }^{45}$ Also in 2015, Rivzi et al reported that patients whose tumors had higher somatic nonsynonymous mutational burden by WES had more durable clinical responses to treatment with pembrolizumab. ${ }^{46}$ Durable clinical benefit (DCB) was defined as a complete or partial response or stable disease for 6 or more months. In their study, $73 \%$ of patients whose tumors had a high TMB (above the median) had DCB, whereas only $13 \%$ of those with low TMB exhibited a benefit $(P=.04)$.

For patients with advanced NSCLC, high TMB assessed with targeted large gene panels also seems to predict durable clinical benefit with PD-1 or PD-L1 blockade. ${ }^{47}$ In this series, the benefit was associated with a TMB above the 50th percentile of the study cohort and was independent of PD-L1 IHC findings. Moreover, other findings, including variants in EGFR or STK11, mitigated 
the benefit of immunotherapy. Further, patients with high TMB and strong IHC staining for PD-L1 fared the best.

TMB also added predicted response beyond PD-L1 status in the CheckMate 026 (NCT02041533) clinical trial, in which 541 patients with previously untreated stage IV NSCLC and a PD-L1 tumor expression of at least $1 \%$ were randomly assigned to receive either nivolumab or standard-ofcare chemotherapy. OS was similar in both cohorts, although the toxicity profile was better for the group of patients receiving nivolumab. ${ }^{48}$ Post hoc analysis showed that the subset of patients with both PDL1 expression more than $50 \%$ and high TMB had a greater likelihood of response to nivolumab, faring better than patients whose tumors exhibited only 1 or neither of these characteristics. Also of note, Chang et al retrospectively assessed TMB in 44 of the patients in this study using the FoundationOne $\mathrm{CDx}$ assay and compared this with the findings obtained from WES. ${ }^{49}$ A very strong correlation (Spearman's $r=$ 0.90 ) existed between the results obtained from both methods. The CheckMate 227 study (NCT02477826) demonstrated that patients fared better with dual ICI using nivolumab and ipilimumab than they did with chemotherapy. ${ }^{50}$ Improved overall tumor response and survival in NSCLC was seen in patients regardless of whether their tumors had more or fewer than 10 mut/ $\mathrm{Mb}$ sequenced using the FoundationOne CDx assay.

Not all studies support TMB as an independent biomarker predictive of a favorable response to ICI. Most do, however, support the predictive value of TMB, and recently published reviews and meta-analyses conclude that TMB is a valid biomarker. $37,51,52$ In April 2020, the FDA granted Fast Track designations for immunotherapeutic agents for metastatic or recurrent NSCLC as well as for urothelial, cervical, and central nervous system cancers based on a high TMB. In addition, in June 2020, the FDA approved pembrolizumab for children and adults with high TMB ( $\geq 10 \mathrm{mut} / \mathrm{Mb})$ solid tumors.

\section{OtherTumors}

The recent FDA approvals for immunotherapy in advanced renal cell carcinoma, and interest in this application, have led to clinical trials that compare different treatment options and the optimal biomarkers for immunotherapy. Somatic mutation data analysis from TCGA in 366 clear cell renal cell carcinoma tumors showed that higher TMB was associated with poor survival in this cohort. The most frequent somatic mutations were in VHL, BAP1, PBRM1, and SETD2 genes. ${ }^{53}$ In the CheckMate 214 study (NCT02231749), patients with renal cell carcinoma were randomized to take the combination of nivolumab plus ipilimumab or monotherapy with sunitinib; $41 \%$ in the combination arm achieved an objective response compared with $34 \%$ in the sunitinib arm. ${ }^{54}$ In another study, KEYNOTE-426 (NCT02853331), the combination of axitinib and pembrolizumab was compared with sunitinib; the objective response was $59.3 \%$ with the combination vs $35.7 \%$ with sunitinib. ${ }^{55}$

The relationship between TMB and response to immunotherapy in bladder cancer was analyzed based on tumor immune microenvironment and tumor genome. Patients with high TMB in their tumors had significantly improved survival compared with the low-TMB group. ${ }^{56}$ Another research group assessed the association between TMB and prognosis in bladder cancer through TCGA data analysis, which showed that patients with high TMB have a better prognosis with immunotherapy than those with low TMB. ${ }^{57}$ As reported by Thomas et al in 2018, patients with breast cancer whose tumors show high TMB have longer survival. ${ }^{58}$ In a study of patients with colorectal cancer (CRC), however, low TMB correlated with favorable PFS but the association did not reach statistical significance. ${ }^{59}$ Finally, in a large pooled analysis of more than 10,000 patients with various tumor types receiving immuno-oncology treatments, authors assessed the relationship between TMB and clinical outcome, concluding that patients with high TMB had better OS when they received immunotherapy versus chemotherapy regardless of tumor type. ${ }^{60}$

\section{Mismatch Repair Deficiency, Hypermutation, and Immuno- Oncology}

Hypermutated tumors appear to be responsive to treatment with immuno-oncology agents. Hypermutation occurs when proofreading mechanisms of DNA replication are impaired, resulting in an accumulation of large numbers of replication errors. DNA mismatch repair would normally correct most of these errors; however, some patients have defects in MMR genes. Lynch syndrome, for instance, is characterized by an autosomal inherited heterozygous mutation in one of the MMR genes: MLH1, MSH2, MSH6, and PMS2. ${ }^{61}$ Lynch syndrome may also be due to the deletion of the 3' exons of the TACSTD1 gene encoding EPCAM. ${ }^{62} \mathrm{MSI}-\mathrm{H}$ could be caused by hypermethylation of the MLH1 promoter ${ }^{63}$ Hypermutation may also be observed in the setting of germline or somatic mutations in the DNA POLE or POLD1 genes. ${ }^{64,65}$ Patients with Lynch syndrome are at an increased risk for CRC, particularly at young age, as well as for cancers of the endometrium, ovary, stomach, small bowel, pancreas, biliary tract, ureter, renal pelvis, brain, and sebaceous glands. ${ }^{66}$

Because dMMR CRCs have high TMB, a trial using pembrolizumab was conducted in 41 patients, including 32 with metastatic CRC. Patients were treated with pembrolizumab (10 mg/kg of body weight) every 14 days. The overall response rate (ORR) was $40 \%$ in patients with $\mathrm{dMMR}$ tumors and $0 \%$ for those whose tumors were MMR proficient. PFS was associated with high TMB $(P=.02)$. WES showed a mean of 1782 somatic mutations in MSI-H/dMMR tumors compared with only 73 observed in MMR-proficient tumors. ${ }^{67} \mathrm{~A}$ subsequent study has validated the observation across multiple tumor types, with a $53 \%$ response rate and $21 \%$ complete response rate seen in patients with advanced dMMR tumors. ${ }^{68}$

In May 2017, the FDA approved pembrolizumab for the treatment of tissue-ag- 
nostic metastatic or unresectable MSI-H/ $\mathrm{dMMR}$ cancers that had progressed on prior treatment or for which no satisfactory treatment options exist. The approval was based on several studies involving patients with 15 different cancer types, including 149 patients with CRC, and it was the first cancer-site-agnostic FDA approval. One of the studies leading to this approval was the important KEYNOTE-016 trial (NCT01876511), ${ }^{67}$ and another was KEYNOTE-158 (NCT02628067), which enrolled 233 patients with 27 different non-CRC cancer types that were all MSI-H/ dMMR. ${ }^{69}$ The ORR to treatment with pembrolizumab was $34 \%$, and one-third of the responding patients had a complete response. Furthermore, $77.6 \%$ of these had response durations of more than 24 months. ${ }^{48}$ In an analysis of cBioPortal data, patients with mutations in POLE or POLD1 across all tumor types who were treated with ICIs had improved OS compared with patients who did not harbor one of these mutations. In the study's multivariable Cox regression analysis controlling for MSI status and tumor type, the presence of a POLE or POLD1 mutation was an independent predictor of a benefit for ICI treatment $(P=.047$; HR, $1.41 ; 95 \% \mathrm{CI}$, $1.00-1.98) .^{70}$

\section{Emerging Biomarkers and Future Directions}

As our knowledge evolves and grows, we will be better able to understand the biomarkers that inform the response to immunotherapy agents. We already understand that certain mutations may inhibit ICI. In nonsquamous NSCLCs that are EGFR and $A L K$ wild type, mutations in $S T K 11 / L K B 1$ predict primary resistance to pembrolizumab, in that the addition of this agent did not improve outcomes associated with platinum doublet chemotherapy. ${ }^{71}$ This finding supports the lack of immuno-oncology drug benefit in KRAS-mutated lung adenocarcinomas with $S T K 11 / L K B 1$ comutations. The STK11/LKB1 gene encodes a serine-threonine kinase that, when inactivated through mutation, is associated with an immunologically inert (cold) tumor immune microenvironment with decreased infiltration of $\mathrm{CD}^{+} \mathrm{T}$ lymphocytes. ${ }^{72,73}$ Lung cancers that exhibited both KRAS and STK11/LBK1 mutations were most resistant to PD-1 ICI, with only $7.4 \%$ of patients deriving a response. ${ }^{74}$ In this study, patients with wild-type STK11/LBK1 tumors that harbored KRAS mutations had an ORR of $28.6 \%$. The investigators also noted that tumors with a STK11/LBK1 mutation were more likely to be PD-L1-negative, although patients with PD-L1-positive, STK11/LBK1-mutated tumors still failed to respond to PD-1/PD-L1 blockade.

Other genomic events also appear to result in acquired resistance to immune therapy. For example, biallelic loss of PTEN was first reported in a patient with metastatic uterine leiomyosarcoma who had a complete remission on pembrolizumab but then developed an acquired resistance. ${ }^{75}$ This has been proposed as a mechanism of resistance in melanoma as well. $^{76}$ Also in melanoma, loss of $B 2 M^{77}$ and loss-of-function mutations of the JAK1 or JAK2 2 genes $^{78}$ are thought to be mechanisms of acquired resistance. In a study by Zaretzki et al, 4 patients with melanoma had initial responses to immunotherapy with pembrolizumab but then developed late resistance. Samples of their tumors from before and after treatment were evaluated by whole exome and transcriptome sequence analysis. It was found that one patient developed a nonsense inactivating mutation in the JAK1 gene that was not seen in the original tumor sample. Another patient's tumor had acquired a splice site variant in the gene encoding JAK2. RNA sequencing confirmed that both mutations resulted in a truncated protein lacking the kinase domain. This makes sense, considering that JAK 1 and JAK2 gene products are thought to be necessary for interferon signaling and response. ${ }^{79} \mathrm{~A}$ third patient developed a frameshift deletion in $B 2 M$, resulting in impaired MHC class I cell membrane localization. No acquired mutation could be identified in the fourth patient studied. The study suggests that
$J A K 1$ and $J A K 2$ loss-of-function mutations may confer resistance to ICI in other tumor types as well. ${ }^{80}$

Studies such as these, with clear biologic correlates, will continue to be important to improve the understanding of how genomic events modulate tumor immunogenicity. We know that not all mutations are equally immunogenic; only a minority of somatic mutations result in neoantigens that are presented in the context of MHC to activate T-cells. ${ }^{81,82}$ Knowing a patient's HLA type and peptide binding motifs allows for predictions of mutated tumor-derived peptides, which can also inform the potential immunogenicity of tumors. This may be even more relevant when combining cellular therapy and immuno-oncology with several agents currently in the pipeline. ${ }^{83}$ Different types of available cellular-based treatments include CRISPR-engineered T-cells or $\delta \delta \mathrm{T}-$-cells, chimeric antigen receptor (CAR) T-cells, and macrophage-based therapies; most of these treatments are autologous.

In addition to the markers that can be identified through tumor testing, additional biomarkers from peripheral blood can and, some research studies suggest, should be assessed, although the value of some is still unclear. For instance, interferon- $\gamma$ is overexpressed and is associated with clinical response in patients with melanoma, but the same result was not reported in NSCLC or renal cell carcinoma. ${ }^{84}$ Bioinformatics tools and high-dimensional single-cell mass cytometry were used to analyze and characterize a subset of immune cells in patients with advanced-stage melanoma before and after immunotherapy. The investigators reported that the frequency of circulating monocytes, CD14+CD16-HLA-DR ${ }^{\text {hi }}$, was a strong predictive factor in the success or failure of immunotherapy. ${ }^{85}$ High-resolution assays of the peripheral immune response and tumor microenvironment may one day enable us to fully apply the precision medicine paradigm to immuno-oncology. These assays include single-cell RNA sequencing, mass cytometry time-of-flight (CyTOF), high-resolution T-cell receptor repertoire sequencing, and tumor DNA WES. For 
instance, Subrahmanyam et al used CyTOF to find predictive markers for anti-PD-1 and anti-CTLA-4 profiling in peripheral blood from patients with melanoma. The authors concluded that $\mathrm{CD}^{+}$and $\mathrm{CD}^{+}$ memory T-cells are important in response to anti-CTLA-4 therapy and could be potential biomarkers. ${ }^{86}$

A gut microbiome profile could be another indication of response to treatment. For example, Akkermansia muciniphila presence in stool samples is associated with a better outcome in NSCLC and renal cell carcinoma. ${ }^{87}$ It is unclear whether manipulation of the microbiome might improve response to immunotherapy. Furthermore, the ratio of neutrophil to lymphocyte and absolute lymphocyte count could be measured before treatment in patients with metastatic disease to assess response to ipilimumab. A high neutrophil/lymphocyte ratio $(\geq 4)$ is an indication of poor prognosis. ${ }^{88}$ Epigenetic markers could also potentially be a prognostic marker in immunotherapy. Investigation of circulating microRNA as a predictive marker in NSCLC showed a 7-miR signature that is associated with outcome in patients who were treated with nivolumab. ${ }^{89}$ Many of these assays, and the advanced bioinformatic algorithms that produce the assay outputs, have not yet been put through the rigorous validation procedures necessary for deployment into the clinical laboratory (Table).

Further, novel imaging studies to monitor effector T-cell trafficking may find a place in the rapid assessment of treatment efficacy. One such modality developed by ImaginAb (Inglewood, CA) uses immunoPET scans with $\mathrm{Zr}$-89 radiolabeled antibodies to $\mathrm{CD}^{+}$T-cells to assess immune-cell trafficking against tumor cells. To date, a phase 1 dose-escalation study demonstrated safety and established the effective dose and the optimal PET imaging protocols. Additional studies, including a phase 2 trial, are ongoing to demonstrate the utility of CD8 immunoPET as a predictive marker for immunotherapy. Once validated, implementing these assays and imaging modalities into routine clinical practice will bring new practical challenges in interpreting the results of, and acting on, these immune assays.

\section{Discussion}

Numerous challenges remain in immuno-oncology, including the determination of the drivers of cancer immunity, preclinical model design, early-phase trials, immune escape, and many more as elucidated by Hedge et al (2020). ${ }^{2}$ Currently, however, the methods to determine if immunotherapy would be effective are limited to PD-1/ PD-L1 expression, MSI, MMR, and TMB status through IHC and next-generation sequencing, with the preference of paraffin-embedded tumor samples. When tumor sample is not readily available, or perhaps for sequential monitoring, assessment of TMB from ctDNA in plasma samples is possible and appears to correlate with findings seen from the analysis of tumor samples by WES. ${ }^{90}$ However, the optimal determination of TMB remains the WES of paired tumor and normal germline DNA in order to call true somatic variants. As technology evolves and expressed variants can be determined, transcriptome analysis may supplant exome sequencing. Furthermore, bioinformatics techniques will continue to improve, allowing for improved analytics.

Other novel immunotherapy agents are in the pipeline, under investigation to target different biomarkers. For example, a coinhibitory molecule called TIGIT-T-cell immunoreceptor with immunoglobulin and immunoreceptor tyrosine-based inhibitory motif domain-is involved in immunosurveillance and is upregulated in certain cancers. Anti-TIGIT antibodies are in the pipeline as immunotherapy agents, to be used as monotherapy or in combination with anti-PD-1/PD-L1 antibodies; they have shown synergy when combined. ${ }^{91}$ Poliovirus receptor-related immunoglobulin domain-containing protein (PVRIG) is another immuno-inhibitory receptor where antagonist antibodies to inhibit PVRIG is currently in development. COM701 is an anti-PVRIG antibody that is currently under investigation. ${ }^{92}$

Evidence is clearly driving the field toward increasingly utilizing high-complexity laboratory testing to help select and monitor patients being treated with immunotherapeutics. A deep understanding of different mechanisms in immune response, and of potential biomarkers to assess sensitivity to immunotherapy, is crucial in the continuing effort to drive clinical benefit in patients with cancer.

FINANCIAL DISCLOSURE: The authors have no significant financial interest in or other relationship with the manufacturer of any product or provider of any service mentioned in this article.

For reference list, visit cancernetwork.com/biomarkers

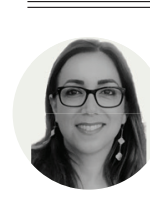

\section{Darabi}

is a clinical genomics scientist for the Precision Medicine Program at Hoag Family Cancer Institute.

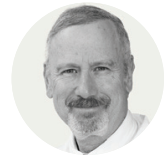

\section{Eisenberg}

is the executive medical director of the Hoag Family Cancer Institute and a professor of clinical surgery at the University of Southern California Keck School of Medicine.

\section{Braxton}

is a molecular pathologist for the Precision Medicine Program at Hoag Family Cancer Institute.

\section{Demeure}

is the program director for the Precision Medicine Program at Hoag Family Cancer Institute. 


\section{Five Ways to Prepare Your Patients forTheir First Telemedicine Visit}

Peter Alperin, MD

$\mathbf{T}$ elemedicine has fast become an important part of our new normal as the need to provide continuity of care to patients with chronic conditions in the face of a global pandemic continues to grow. Moving forward, there will be a significant number of patients for whom it will not make sense to have to physically come into a doctor's office when they can get the same level of care by simply doing a video visit with their physician. This, in part, accounts for the meteoric rise of telehealth and telemedicine.

Many in the medical community are starting to realize that the broad adoption of telemedicine across the system could be a great equalizer for communities that have historically struggled to access care-remote rural or underserved urban communities. There's great hope that telemedicine could be one development that helps bridge a persistent divide in outcomes, as long as accessing it is easy and inexpensive for patients.

It's understandable that some patients may view telemedicine as a challenge, either because of their lack of confidence with technology or not feeling that they have the resources to connect with their doctor remotely. As a practicing internist, I've had the privilege of helping patients with the basics of getting started with their first telehealth appointment.
Here are 5 things you can tell your patients to do to help them prepare for their first virtual visit:

1. TEST YOUR TECH: Virtual visits will require a basic familiarity with videoconferencing on a tablet or smartphone. Though the device patients use is up to them, it's important that their internet connection-either Wi-Fi or cellularis fast enough to do the call. Urge your patients to give it a test before their first appointment. Depending on the platform you use, your patient may need to have an app already downloaded on their phone and be registered as a new user. Every platform is different, so make sure to tell your patient in advance if there are any special requirements.

2. PREPARE QUESTIONS IN AdVANCE: Telemedicine visits are typically about $20 \%$ shorter than in-person appointments. As with any in-person doctor visit, patients will want to maximize their time by preparing questions they have in advance of the appointment. Encourage them to set an agenda and have questions handy during the call to ensure they get everything answered.

\section{CHECK COVERAGE AND CO-PAYS WITH YOUR} INSURANCE PROVIDER: Efforts by insurance providers to update coverage policies amid the surge in telemedicine have created a patchwork of policies that isn't always clear. Have your patients contact your organization before their first telemedicine visit to double-check what's covered under their plan.

4. GET COMFORTABLE: Telemedicine visits are subject to HIPAA (the Health Insurance Portability and Accountability Act) and are private matters, so make sure your patients are aware and have a quiet, discreet space in which to take the call and use headphones or earbuds if possible. Good lighting is also important for any visible concerns they may have. Ask them to wear clothing that is easy to move in so they are best able to participate in a physical exam.

\section{BE PREPARED TO TALK ABOUT FOLLOW-UP}

CARE: Before ending the video call, make sure that patients understand clear next steps (if applicable). They should have a clear understanding of how to get in touch with you and understand where and how to get necessary prescriptions. They can always check in with the administrative staff of your office after your telehealth visit if they need written paperwork for follow-up purposes.

Peter Alperin, MD, is a practicing internist and vice president at Doximity.

\section{FINANCIAL DISCLOSURE: The authors have} no significant financial interest in or other relationship with the manufacturer of any product or provider of any service mentioned in this article. 


\section{CONTINUING MEDICAL EDUCATION (CME)}

\section{Changing Landscape in MDS}

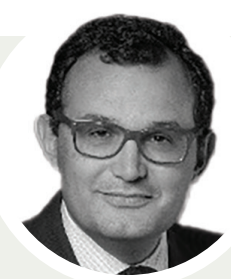

FACULTY

Guillermo Garcia-Manero, MD McCredie Professor of Medicine Chief, Section of MDS

Vice Chair, Dept. of Leukemia

University of TexasMD Anderson Cancer Center

This activity was written by $P E R^{\circledR}$ editorial staff under faculty guidance and review. The Q\&A portion of the activity was transcribed from a recorded interview with the faculty and edited by faculty and $P E R^{\circledR}$ editorial staff for clarity.

\section{CME PROVIDER CONTACT} INFORMIATION

Physicians' Education Resource ${ }^{\circledR}$, LLC 2 Clarke Drive, Suite 110

Cranbury, NJ 08512

Toll-Free: 888-949-0045

Local: 609-378-3701

Fax: 609-257-0705

info@gotoper.com

\section{LEARNING OBJECTIVES}

Upon successful completion of this activity, you should be better prepared to:

- Describe how current classification and risk-stratification of myelodysplastic syndromes (MDS) guides clinical decisions.

- Assess the role of individual patient characteristics on prognosis, including response to treatment, clinical outcomes, and progression to acute myeloid leukemia.

- Evaluate how investigational and emerging therapeutic strategies for MDS could be integrated in therapeutic regimens.

RELEASE DATE: August 1, 2020

EXPIRATION DATE: August 1, 2021

\section{INSTRUCTIONS FOR PARTICIPATION /} HOW TO RECEIVE CREDIT

1. Read this activity in its entirety.

2. Go to https://www.gotoper.com/go/onc-mds20print to access and complete the posttest.

3. Answer the evaluation questions.

4. Request credit using the drop-down menu.

You may immediately download your certificate.

FACULTY, STAFF, AND PLANNERS' DISCLOSURES

In accordance with ACCME Guidelines, PER ${ }^{\circledast}$ has identified and resolved all COl for faculty, staff, and planners prior to the start of this activity by using a multistep process.

Disclosures (Dr. Guillermo Garcia-Manero): Grant/Research Support: BMS, Novartis, Taiho

The staff of $P E R^{\circledast}$ have no relevant financial relationships with commercial interests to disclose.

OFF-LABEL DISCLOSURE AND DISCLAIMER

This activity may or may not discuss investigational, unapproved, or off-label use of drugs. Learners are advised to consult prescribing information for any products discussed. The information provided in this activity is for accredited continuing education purposes only and is not meant to substitute for the independent clinical judgment of a healthcare professional relative to diagnostic, treatment, or management options for a specific patient's medical condition. The opinions expressed in the content are solely those of the individual faculty members, and do not reflect those of PER ${ }^{\circledR}$ or any of the companies that provided commercial support for this activity.

This activity is funded by PER ${ }^{\circledast}$.

ACCREDITATION/CREDIT DESIGNATION

Physicians' Education Resource ${ }^{\circledast}$, LLC, is accredited by the Accreditation Council for Continuing Medical Education (ACCME) to provide continuing medical education for physicians. Physicians' Education Resource ${ }^{\circledast}$, LLC, designates this enduring material for a maximum of 0.5 AMA PRA Category 1 Credits $^{m}$. Physicians should claim only the credit commensurate with the extent of their participation in the activity. 
$\mathrm{M}$ yelodysplastic syndromes (MDS) are a group of clonal myeloid neoplasms characterized by ineffective hematopoiesis, risk of clonal evolution and progression to acute myeloid leukemia (AML), abnormal blood and marrow cell morphology and cytopenias. Although MDS shares clinical and pathological features with AML, MDS has a lower percentage of blasts in peripheral blood and bone marrow than AML. The cause of the mutations in hematopoietic stem cells that give rise to MDS is unknown, although in some cases the mutations are due to prior exposure to cytotoxic chemotherapy and/or ionizing radiation, or due to exposure to environmental toxins..$^{1-5}$ More than $90 \%$ of cases of MDS harbor detectable driver mutations, including mutations in DNMT3A, EZH2, RUNX1, TET2, IDH1, IDH2, TP53, ASXL1 and mutations in genes encoding components involved in 3' RNA splicing. ${ }^{5}$

MDS has a highly heterogeneous clinical presentation. Not all patients with MDS present with fatigue, infections, or other symptoms related to cytopenias. In some asymptomatic patients, MDS is only detected via abnormal results on a routine complete blood count. Anemia is the most common cytopenia, and the fatigue associated with anemia may be disproportionate to the degree of anemia. ${ }^{5,6}$ Other symptoms, such as leukopenia-associated or thrombocytopenia-associated bleeding may be present. ${ }^{5}$

\section{Diagnosis and Risk Stratification of MDS}

Diagnosis of MDS is based on morphological evidence of dysplasia via visual assessment of a bone marrow aspirate and biopsy, with the use of additional complementary studies such as flow cytometry, karyotyping, or molecular genetics that support and refine diagnosis. ${ }^{7}$ Risk stratification of MDS via the International Prognostic Scoring System (IPSS) was originally based on a score compiled from summing the scores that range from 0 to 2 in each of the 3 variables of bone marrow blast percentage, karyotype, and cytopenias. ${ }^{8}$ Risk groups were defined as low ( 0 points), intermediate- 1 (0.5-1.0 points), intermediate- 2 (1.5-2.0 points), and high (2.53.5 points). ${ }^{8}$

The revised IPSS (IPSS-R) stratifies for risk based on 5 variables. ${ }^{9}$ Different numbers of points are assigned to each variable, with bone marrow blast percentage (3 points), karyotype (4 points), hemoglobin (1.5 points), platelets (1 point), and absolute neutrophil count ( 0.5 points) used as the variables for stratification. The summation of points from each category defines 5 risk groups for both overall survival and evolution to AML. ${ }^{9}$ The risk categories for IPSS-R are very low ( $\leq 1.5$ points), low ( $>1.5$ to 3 points), intermediate ( 3 to 4.5 points), high ( $>4.5$ to 6 points), and very high (> 6 points). ${ }^{9}$

IPSS-R incorporates a cytogenetic MDS score and different cutoff points for cytopenias than IPSS and should be used to evaluate risk. However, no therapeutic regimens have been approved using IPSS-R. ${ }^{7}$ Additionally, IPSS-R was developed in patients with newly-diagnosed MDS at initial presentation and not in patients who develop MDS related to prior treatment with chemotherapy and/or radiotherapy. ${ }^{7}$

Additional prognostic models could provide increased resolution to evaluating risk. The global MDACC model enables risk assessment of all patients with MDS at any point in the course of their disease. ${ }^{710}$ Additional challenges to determining prognosis in MDS include the observation that patients with lower-risk disease often experience a highly heterogeneous disease course, and the presence of comorbidities can affect survival in older patients who are diagnosed with MDS. ${ }^{7}$

Mutations in DNMT3A, EZH2, RUNX1, TET2, IDH1, IDH2, TP 53, and ASXL1 have been associated with worse prognosis in MDS while mutations in SF3B1, a gene encoding a splicing factor, have been associated with better prognosis. ${ }^{5,7}$ The presence of different mutations could also potentially predict response to treatment. For example, patients with TET2 mutations may have higher response rates to azacitidine than patients without TET2 mutations. ${ }^{7}$

\section{Current Treatment Regimens for MDS}

Current treatment for MDS relies primarily on risk-adapted therapy from IPSS. ${ }^{7}$ In patients with newly-diagnosed lower-risk MDS, treatment is based on whether the patient requires transfusions. In transfusion-independent patients, observation is common until they become dependent on transfusion. For patients with lower-risk MDS, treatment typically proceeds from the use of growth factor inhibitors to lenalidomide to a hypomethylating agent (HMA; azacitidine or decitabine). If these patients fail all 3 therapies, they should be considered for allogeneic stem cell transplant (alloSCT) and/or clinical trials. ${ }^{7}$ Patients with higher-risk MDS can undergo alloSCT when eligible, receive an HMA, and receive treatment normally administered to patients with AML. Prognosis is poor in higher-risk patients who fail any of these lines of therapy but are particularly worse in patients who fail an HMA. AlloSCT and/or enrollment in a clinical trial should be considered. ${ }^{7}$

Some therapeutic regimens in MDS are off-label. Erythropoiesis-stimulating agents (ESAs) such as epoetin and darbepoetin are used extensively off-label in patients with MDS to address anemia. Because the National Comprehensive Cancer Network (NCCN) guidelines support the use of ESAs in patients with MDS, it is reimbursed by Medicare as compendium use. ${ }^{11}$ The BCL-2 inhibitor venetoclax is under clinical evaluation in MDS and has not been approved for use. Nonetheless, it is sometimes used off-label in the second-line setting in patients who failed an HMA. ${ }^{11}$

Supportive care in MDS includes treatment beyond transfusions. Since infection is the leading cause of death 
in MDS, fevers should be taken seriously, although the role of antimicrobials when febrile neutropenia is present is controversial. ${ }^{11}$ Patients with thrombocytopenia refractory to platelet transfusions may require additional therapy, which can include antifibrinolytic agents. Additionally, patients who receive repeated red blood cell transfusions may require iron chelation. ${ }^{11}$

In April 2020, the United States Food and Drug Administration (FDA) approved the erythroid maturation agent luspatercept for the treatment of very low- to intermediate-risk MDS patients who fail an ESA and require 2 or more red blood cell units over 8 weeks. ${ }^{12}$ The approval was based on results from the phase 3, double-blind MEDALIST trial (NCT02631070) that demonstrated that luspatercept reduced the severity of anemia in patients with lower-risk MDS who had been receiving regular transfusions and had disease that was refractory to, or unlikely to respond to ESAs, or had discontinued ESAs due to adverse events (AEs). ${ }^{13}$ In the trial, 153 patients were randomly assigned to receive luspatercept, and 76 were randomly assigned to receive placebo. The primary endpoint was achieving transfusion-independence for 8 weeks or longer, which occurred in 38\% of patients in the luspatercept arm compared with $13 \%$ of patients in the placebo arm $(P<.001)$. More patients in the luspatercept arm met the key secondary endpoint of transfusion-independence for 12 weeks or longer. ${ }^{13}$ At week
$24,28 \%$ of patients in the luspatercept arm were transfusion-independent compared with $8 \%$ in the placebo arm $(P<.001)$. This difference remained significant through week 48 , when $33 \%$ of patients in the luspatercept arm and $12 \%$ of patients in the placebo arm were transfusion-independent $(P<.001) .{ }^{13}$ The most frequently occurring all-grade AEs in $10 \%$ or more of patients with luspatercept or placebo were fatigue $(27 \%$ and $13 \%$, respectively), diarrhea (22\% and $9 \%$ ), asthenia $(20 \%$ and $12 \%)$, nausea $(20 \%$ and $8 \%$ ), dizziness (20\% and $5 \%$ ), and back pain (19\% and $5 \%$ ). Grade 3 or 4 AEs occurred in $42 \%$ of patients receiving luspatercept and $45 \%$ of patients receiving placebo during the trial. Serious AEs occurred in $31 \%$ of patients receiving luspatercept and $30 \%$ of patients receiving placebo. Dose reductions occurred in 5\% of patients receiving luspatercept and no patients receiving placebo. ${ }^{13}$

In July 2020, the FDA approved oral tablets of decitabine with cedazuridine (ASTX727) to treat MDS based on results from the phase 3,2-sequence crossover ASCERTAIN trial (NCT03306264). ${ }^{14}$ The trial achieved its primary endpoint of systemic exposure equivalence between ASTX727 and IV decitabine with an oral/ IV ratio of around $99 \%$ in patients with intermediateand high-risk MDS. The most common AEs occurring in $\geq 20 \%$ of patients included fatigue, constipation, hemorrhage, myalgia, mucositis,

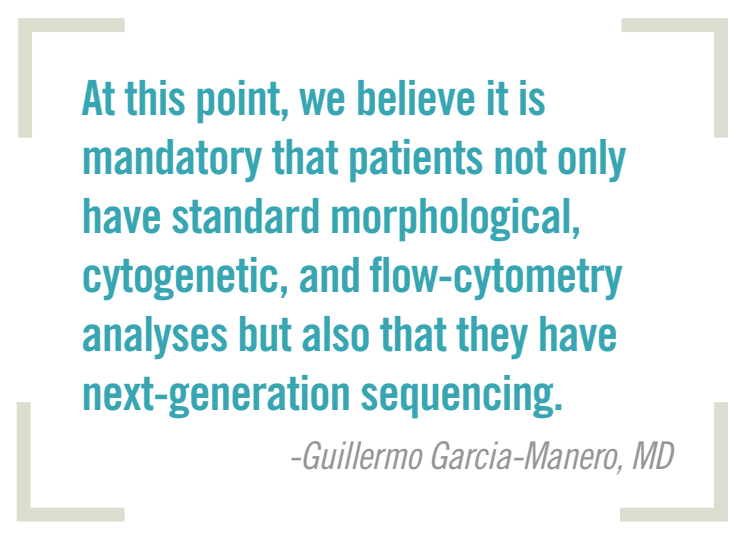

arthralgia, nausea, dyspnea, diarrhea, rash, dizziness, febrile neutropenia, edema, headache, cough, decreased appetite, upper respiratory tract infection, pneumonia, and transaminase increase. Serious AEs that occurred in more than $5 \%$ of patients were febrile neutropenia, pneumonia, and sepsis. The safety profiles were similar for the oral vs IV formulations. ${ }^{14}$

\section{Emerging and Investigational Therapies in MDS}

Emerging and investigational therapies in MDS harbor the promise of advancing therapeutic options in these patients. The development of new therapies is particularly important for patients who fail treatment with HMAs. Although HMAs are the standard-of-care treatment for patients with MDS, only approximately $50 \%$ of patients respond to them, and responses are typically transient, with loss of response often occurring within 2 years. ${ }^{15}$ Loss of response to HMAs is correlated with poor prognosis, and subsequent lines of therapy are limited..$^{15}$

The oral Polo-like kinase 1 inhibitor rigosertib was combined with azacitidine in a phase $1 / 2$ trial in patients with MDS who were naïve to HMAs and in patients who had failed HMAs. Of the 55 patients evaluable for response, median duration of response was 12.2 months in HMAnaïe patients and 10.8 months in HMA-failure patients. ${ }^{16}$ Responses to treatment occurred in all IPSS-R subgroups. In the low/intermediate-risk subgroup ( $\mathrm{n}=17$ ), complete response (CR) occurred in 4 patients and marrow CR (mCR) occurred in 5 patients. In the high-risk subgroup ( $\mathrm{n}=$ 23), CR occurred in 2 patients, partial response (PR) in 1 patient, and $\mathrm{mCR}$ in 8 patients. In the very high-risk subgroup ( $\mathrm{n}=33)$, CR occurred in 5 patients and $\mathrm{mCR}$ in 10 patients. ${ }^{16}$ Four safety optimization strategies were used to minimize genitourinary toxicities, including taking rigosertib at least 2 hours after lunch to avoid nocturnal bladder dwell time, encouraging oral hydration of at least 2 liters of fluid per day, mandatory bladder emptying before bedtime, and assessing urine $\mathrm{pH}$ around 2 hours after the morning dose of rigosertib. ${ }^{16}$ 
Immune checkpoint inhibitors both as monotherapy and in combination with other therapies are being investigated in MDS. Trials with the anti-PD1 monoclonal antibody pembrolizumab in patients with HMA-treatment failure, resulted in no CRs, $1 \mathrm{PR}$, an overall response rate (ORR) of $4 \%$, and a 24 -week OS rate of $49 \%$. In total, $36 \%$ of patients experienced treatment-related AEs, most of which were grade 1 or $2 .{ }^{15,17} \mathrm{~A}$ phase 2 trial of the anti-CTLA-4 monoclonal antibody ipilimumab vs the anti-PD1 monoclonal antibody nivolumab in 35 patients with MDS that failed to respond to HMAs showed an OS of 8.0 months in both arms, a $15 \%$ $\mathrm{CR}$ in the ipilimumab arm, and no CRs in the nivolumab arm. ${ }^{18}$

The BCL-2 inhibitor venetoclax is being investigated in MDS as a monotherapy and in combination with azacitidine. A phase 1 trial (NCT02966782) is evaluating plasma concentration, clearance, Cmax, and other parameters of venetoclax with or without azacitidine in patients with relapsed or refractory MDS. ${ }^{19}$ Interim results indicated an ORR of $7 \%$, a median PFS of 3.4 months, and an estimated 6 -month OS of $57 \%$ in the venetoclax single-agent arm. In the venetoclax plus azacitidine arm, ORR was 50\% and median PFS and OS were not reached. The most frequent treatment-emergent AEs were neutropenia, thrombocytopenia, nausea, and diarrhea. ${ }^{20}$

The selective NEDD8 inhibitor pevonedistat was combined with azacitidine in a phase 2 trial (NCT02610777) vs single-agent azacitidine in patients with higher risk MDS/chronic myelomonocytic leukemia or low-blast AML. Event-free survival was 21 months in the pevonedistat arm vs 16.6 months in the single-agent pevonedistat arm. The safety profile was comparable between the 2 arms, with grade 3 or higher AE rates of at $90 \%$ vs $87 \%$ in the pevonedistat vs single-agent azacitidine arm. ${ }^{21}$

Finally, novel formulations of HMAs could yield longer half-lives, decreased toxicity, and/or a more manageable route of administration. The July 2020 FDA approval of oral decitabine with cedazuridine is one example of such a novel formulation. ${ }^{14}$ Guadecitabine is another next-generation HMA that is administered subcutaneously with a longer half-life and exposure than traditional decitabine. ${ }^{15}$

In the interview below, Guillermo Garcia-Manero, MD, provides his insights into the current and changing treatment landscape in patients with MDS.

\section{What are the goals of therapy in patients with lower-risk MDS vs higher-risk MDS?}

GARCIA-MANERO, MD: This is a controversial topic. In patients with higher-risk disease, the goal is improvement of survival. In patients with lower-risk disease, this is, again, a little bit controversial. Of course, the main objective for patients with lower-risk disease would be improvement of cytopenias, and hopefully that translates into improvement in quality of life, reduced transfusion needs, less anemia, less thrombocytopenia. But there is an emerging concept that perhaps we can also improve survival in a subset of patients with what we now call low-risk disease that may actually have a relatively short survival. I think that we should also consider improvement of survival as a goal of therapy in these patients.

\section{In which patients with MDS is supportive care important?}

GARCIA-MANERO, MD: Supportive care is important for all patients with MDS. Both high-risk and low-risk patients need transfusion support. They may be a candidate for growth factor inhibitors, and they may need interventions to decrease the risk of infectious complications that we have shown to be one of the most frequent causes of death in patients with this particular disease. ${ }^{11}$ Supportive care is fundamental for all patients with MDS.

\section{How does off-label - use of drugs (eg, venetoclax, ESAs) affect the treatment landscape of MDS?}

GARCIA-MANERO, MD: ESAs, or erythropoiesis-stimulating agents, have been one of the clear scaffolds of both the treatment and the supportive care of patients with MDS, but there are no significant prospective randomized studies showing that they benefit our patients. ${ }^{11}$ However, they are an important part of therapy in patients with MDS, particularly patients with lower-risk disease who are anemic.
Now, the role of venetoclax is more complex. Venetoclax is a very active compound for patients with AML, and it is actively being investigated for patients with high-risk MDS. We should not be using this compound off-label right now in patients with high-risk MDS; instead, we should really learn from these clinical trials that are ongoing and accruing before we make venetoclax the standard-of-care treatment. I say that because I think that eventually we are going to learn that there are subsets of patients that may or may not benefit from this drug, and therefore a better understanding of patients in whom to use venetoclax in is really important. This is particularly important because venetoclax can cause severe cytopenia and may have some unwanted side effects in patients with MDS if we use this drug indiscriminately.

\section{What role might oral hypomethylating agents have in treatment of patients with MDS?}

GARCIA-MANERO, MD: The hypomethylating agents decitabine and azacitidine are our main treatment options for patients with MDS. These IV or subcutaneous injected drugs require multiple visits per month, which can pose a major problem for this patient population that tends to be older. As a result, an oral hypomethylating agent would be a dramatic improvement for our patients.

There are currently 2 different strategies in the development of oral hypomethylating 
agents. One strategy uses an oral form of azacitidine with a lower pharmacokinetic profile than that of regular azacitidine. Therefore, this compound should perhaps be considered a different drug than the original azacitidine, and it should be studied as such.

In contrast, another compound known as ASDX-727, that is basically oral decitabine, has an identical pharmacokinetic profile to that of IV decitabine. ${ }^{14}$ So in that context, ASDX-727 could basically be interchanged with the original decitabine.

This is a very important topic. I believe that the oral hypomethylating agents in time will replace IV and subcutaneous hypomethylating agents, but they will have different implications and potentially different uses. And one last thing that is very important is that many of these compounds we are discussing (eg, venetoclax) are already oral compounds, so we have been discussing the development of total oral therapies for our patients, which will be tremendously facilitated by oral hypomethylating agents.

Another consideration with the oral hypomethylating agents is actually that, because they are oral, they have more flexibility in terms of dose and schedules. This could, for example, allow for attenuated low-dose schedules that we could use in patients with low-risk MDS. I think this is going to give us flexibility and will expand the use of these hypomethylating agents beyond traditional use in MDS and AML.

\section{What are rational combinations for immune checkpoint inhibitors in MDS?}

GARCIA-MANERO, MD: My group and others have shown that hypomethylating agents trigger a PD-1/PD-L1 cascade, and we have been actively investigating the activity of these compounds in MDS, both in the frontline setting and preclinically. ${ }^{15}$ Our data indicate that the anti-CTLA-4 monoclonal antibody ipilimumab could have significant activity in this group of patients, and we are now expanding those clinical trials. ${ }^{18}$ Although these drugs are widely approved for multiple tumors, they are not yet ready for use in patients with MDS outside of clinical trial protocols. We still need larger randomized trials. The main issue with immune checkpoint inhibitors is potential toxicity, especially pneumonitis, that could be complex to treat in these patients with MDS.

\section{How can patients with MDS best be selected for treatment with current, novel, and emerging therapies?}

GARCIA-MANERO, MD: This is a very important topic. At this point, we believe it is mandatory that patients not only have standard morphological, cytogenetic, and flow-cytometry analyses done but that they also have next-generation sequencing analyses done. In our hospital, we perform an 81-gene panel, but different institutions have different but sufficiently similar genomic profiling assays. This is critical to deploy at baseline. We have calculated that if we look at the most common mutations in MDS, we can probably inform close to $40 \%$ of the patients. The genes with actionable interest today are TP53, IDH1, IDH2, FLT3, and NPM1. People may question why I mentioned FLT3, but our data and others indicate that in the relapse setting, a significant fraction of patients, approximately $15 \%$ of them, may actually have a detectable FLT3 mutation at the time of hypomethylating-agent failure. ${ }^{22}$ Detecting these mutations can actually help direct what will potentially be an appropriate, more-effective therapy for this group of patients.

Furthermore, genomic analysis has implications in terms of calculating the prognosis of the patient, regardless of whatever therapy you decide to give, and also, very importantly, it may help us calculate what is the potential outcome with alloSCT. Another important aspect of these genomic assays is that, in our opinion, not only do they need to be performed at baseline but also sequentially. We have observed that many patients who lose response to whatever therapy they are receiving gain mutations in some of these genes that I mentioned earlier; therefore there may be an opportunity for the use of second-line targeted therapies.

Of course, this means that approximately $60 \%$ of patients do not have a genomic lesion that informs treatment. Some of these patients, however, still have cytogenetic abnormalities and can experience improved outcomes if they are treated with an AML-like program.
Cytogenetic and molecular information is crucial to predict prognosis, to select therapy, and to determine the role of stem-cell transplant.

\section{What novel therapeutic targets are there in MDS, and how are they being developed?}

GARCIA-MANERO, MD: Right now, there is a plethora of investigational clinical trials being conducted in MDS. This is starting to result in drugs being approved for patients with MDS.

A study with the anti-CD47 agent magrolimab is near completion and has significant activity in MDS. Ongoing clinical trials are assessing inhibitors of IDH2 and IDH1. We are all anticipating results from studies with venetoclax, both in the frontline and in the relapse settings. These are very important randomized trials that will allow us to actually understand the proper role of venetoclax in high-risk MDS. In addition, multiple agents have completed phase 3 trials or are currently being evaluated. Agents such as rigosertib and the anti-TIM-3 immune-checkpoint inhibitor MBG453 show promise in treating patients with MDS. ${ }^{23}$

\section{How are patients with progressive or refractory disease managed?}

GARCIA-MANERO, MD: This is a major problem because the natural history of the prognosis of patients with progressive or refractory MDS is very poor. When we performed the phase 3 trial of rigosertib, we 
learned that there are 2 types of patients, what we call primary failure, which includes patients who never responded to therapy, and then patients with secondary failure, which includes patients who had a response to the hypomethylating agent but then later lost that response. In this high-risk setting, survival is very short, approximately 4 to 6 months. Induction chemotherapy very rarely works in this context, and transplant does not really have activity unless we can achieve a complete response with the patient.

In this context, assessing mutations and conducting cytogenetic profiling is really, really important to guide subsequent treatment. Patients with specific mutations may be candidates for certain therapies in the context of a clinical trial. That is really how we approach it. Otherwise, there is no good standard-of-care therapy, and the prognosis is poor.

Now, on the other hand, there is also the subset of patients with lower-risk HMA failure. These are not true progressive patients, but rather patients who were treated with a hypomethylating agent [whose therapy failed them.] They do quite well with a stem-cell transplantation, but unfortunately many of these patients are not transplant candidates. Therefore we need new therapies for this patient population. In summary, treatment decisions should be made based on cytogenetic and genomic assessments. There may be some subsets of patients that could benefit from some type of adopted standard-of- care treatment, but in reality, most of these patients should be considered, if possible, for some form of clinical trial.

\section{REFERENCES}

1. Will B, Zhou L, Vogler TO, et al. Stem and progenitor cells in myelodysplastic syndromes show aberrant stage-specific expansion and harbor genetic and epigenetic alterations. Blood. 2012;120(10):2076-2086. doi:10.1182/blood-2011-12-399683

2. Bowen DT. Occupational and environmental etiology of MDS. Best Pract Res Clin Haematol. 2013;26(4):319-326. doi:10.1016/j. beha.2013.09.002

3. Pang WW, Pluvinage JV, Price EA, et al. Hematopoietic stem cell and progenitor cell mechanisms in myelodysplastic syndromes. Proc Natl Acad Sci U S A. 2013;110(8):3011-3016. doi:10.1073/pnas.1222861110

4. Abbas S, Kini A, Srivastava VM, et al. Coexistence of aberrant hematopoietic and stromal elements in myelodysplastic syndromes. Blood Cells Mol Dis. 2017;66:37-46. doi:10.1016/j.bcmd.2017.08.004

5. Hellström-Lindberg E, Tobiasson M, Greenberg P. Myelodysplastic syndromes: moving towards personalized management. Haematologica. 2020;105(7):17651779. doi:10.3324/ haematol.2020.248955

6. Abel GA, Efficace F, Buckstein $\mathrm{RJ}$, et al. Prospective international validation of the Quality of Life in Myelodysplasia Scale (QUALMS). Haematologica. 2016;101(6):781-788. doi:10.3324/ haematol.2015.140335

7. Montalban-Bravo G, GarciaManero G. Myelodysplastic syndromes: 2018 update on diagnosis, risk-stratification and management. Am J Hematol. 2018:93(1):129-147. doi:10.1002/ ajh.24930

8. Greenberg P, Cox C, LeBeau $\mathrm{MM}$, et al. International scoring system for evaluating prognosis in myelodysplastic syndromes. Blood 1997;89(6):2079-2088. Published correction appears in Blood.
1998;91(3):1100

9. Greenberg PL, Tuechler $\mathrm{H}$, Schanz J, et al. Revised international prognostic scoring system for myelodysplastic syndromes. Blood. 2012;120(12):2454-2465. doi:10.1182/blood-2012-03-420489

10. Kantarjian $\mathrm{H}$, O'Brien S, Ravandi F, et al. Proposal for a new risk model in myelodysplastic syndrome that accounts for events not considered in the original International Prognostic Scoring System. Cancer. 2008;113(6):13511361. doi:10.1002/cncr.23697

11. Steensma DP. Myelodysplastic syndromes current treatment algorithm 2018. Blood Cancer J. 2018;8(5):47. doi:10.1038/s41408018-0085-4

12. U.S. Food and Drug Administration (FDA) approves Reblozyl (luspatercept-aamt), the first and only erythroid maturation agent, to treat anemia in adults with lower-risk myelodysplastic syndromes (MDS). Press release. Bristol Myers Squibb. April 3, 2020. Accessed June 16, 2020. https:// news.bms.com/press-release/ corporatefinancial-news/us-foodand-drug-administration-fdaapproves-reblozyl-luspater.

13. Fenaux P, Platzbecker U, Mufti GJ, et al. Luspatercept in patients with lower-risk myelodysplastic syndromes. N Eng J Med. 2020;382(2):140-151. doi: 10.1056/NEJMoa1908892

14. FDA Approves At-Home Therapy to Treat Myelodysplastic Syndromes. AJMC website. July 11, 2020. Accessed July 13, 2020. https://www.ajmc.com/newsroom/ fda-approves-athome-therapy-totreat-myelodysplastic-syndromes

15. Gil-Perez A, Montalban-Bravo G. Management of myelodysplastic syndromes after failure of response to hypomethylating agents. Ther Adv Hematol. 2019;10:2040620719847059. doi:10.1177/2040620719847059

16. Navada SC, Garcia-Manero G, Atallah $\mathrm{E}$, et al. Phase II study of oral rigosertib combined with azacitidine in patients with higher-risk myelodysplastic syndromes (MDS). Blood. 2019; 134(suppl_1):566.

17. Ribrag V, Armand P, Kuruvilla $J$, et al. An open-label, multicohort phase $\mathrm{lb}$ trial of pembrolizumab (MK-3475) for advanced hematologic malignancies: KEYNOTE-013. J Immunotherap Cancer. 2015;3(suppl 2):P169. doi:10.1186/2051-1426-3-S2-P169

18. Garcia-Manero G, Sasaki K, Montalban-Bravo G, et al. A phase II study of nivolumab ipilimumab with or without azacitidine for patients with myelodysplastic syndrome (MDS). Blood. 2018;132(Suppl_1):465 Presented at: American Society of Hematology Annual Meeting; November 29, 2018; San Diego, CA. Abstract 465. 19. A study evaluating venetoclax alone and in combination with azacitidine in subjects with relapsed/refractory myelodysplastic syndromes (MDS). ClinicalTrials. gov. Updated June 2, 2020 Accessed July 13, 2020. https:// clinicaltrials.gov/ct2/show/ NCT02966782.

20. Zeidan AM, Pollyea DA, Garcia JS, et al. A phase 1b study evaluating the safety and efficacy of venetoclax as monotherapy or in combination with azacitidine for the treatment of relapsed/refractory myelodysplastic syndrome. Blood. 2019;134(suppl_1):565.

21. Ades L, Watts JM, Radinoff A, et al. Phase II study of pevonedistat $(P)+$ azacitidine $(A)$ versus $A$ in patients (pts) with higher-risk myelodysplastic syndromes (MDS)/ chronic myelomonocytic leukemia (CMML), or low-blast acute myelogenous leukemia (LB AML) (NCT02610777). J Clin Oncol. 2020;38;(suppl 15): abstr 7506).

22. Kubasch AS, Platzbecker $U$. The wolf of hypomethylating agent failure: what comes next? Haematologica. 2019;104(8):15051508. doi:10.3324/ haematol.2019.222794

23. Garcia-Manero G, Navada SC, Fenaux P, et al. Phase 3, multicenter, international, randomized, double-blind, placebo controlled study of oral rigosertib + injectable azacitidine (AZA) versus injectable azacitidine in treatmentnaive patients with higher-risk myelodysplastic syndrome (HRMDS). Blood. 2019;134(suppl 1):4268. 


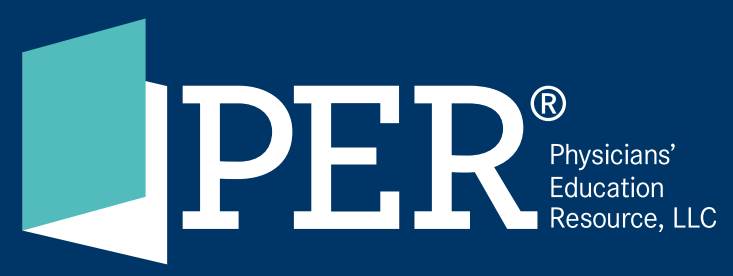

\section{Leading the way in trusted high-impact CME for Oncologists}

When break through data comes to light, $\mathrm{PER}^{\circledR}$ is the go-to-resource

Oncologists can count on for practical strategies to inform the day-to-day care of patients. As the official CME provider for Oncology, $\mathrm{PER}^{\circledR}$ is leading the way with relevant clinical information, renowned faculty, on-demand interactive formats.

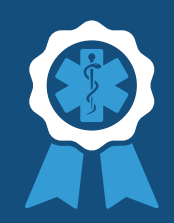

\section{7! is of high-impact CME/CE}

Growing MULTI-PLATFORM Reach live events, interactive webinars, online activities

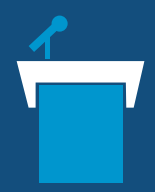

\section{PHYSICIAN COIHRIBUTORS} to $\mathrm{PER}^{\circledast}$ content in 2019

Visit gotoper.com/go/Onc to learn more about our upcoming programs.

Stay up-to-date on the latest breakthroughs in Ophthalmologists with PER ${ }^{\circledR}$ 


\section{COMING SOON \\ OUR NEXT INDICATION}

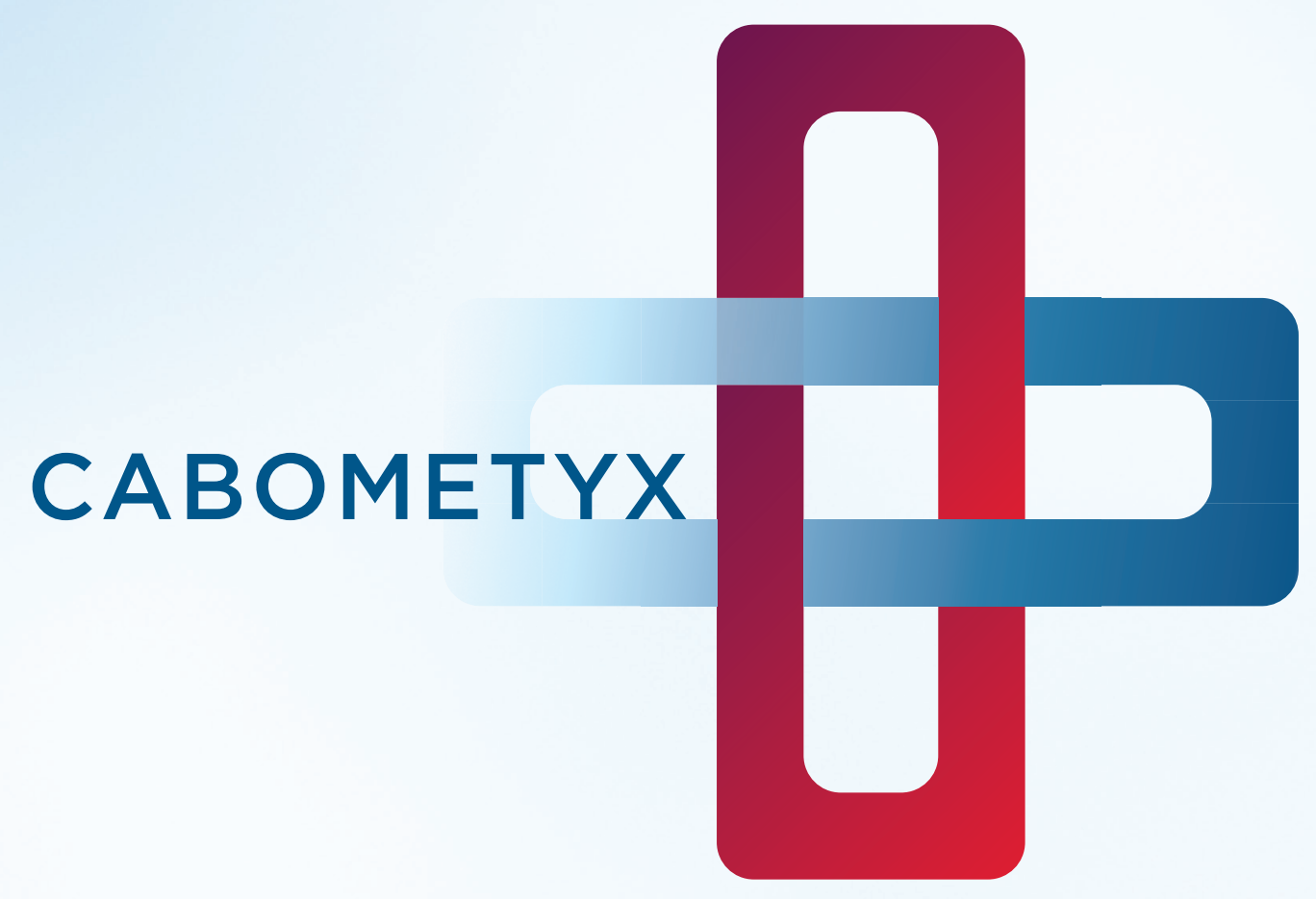

Sign up for updates by scanning the $Q R$ code or visiting CABOMETYXnextindication.com

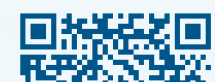
rise 约 